Supporting Information for:

\title{
Using Low-Pressure Methane Adsorption Isotherms for Higher-Throughput Screening of Methane Storage Materials
}

Kyle J. Korman, Gerald E. Decker, Michael R. Dworzak, Meaghan M. Deegan, Alexandra M. Antonio, Garrett A. Taggart, and Eric D. Bloch ${ }^{*}+$

†Department of Chemistry and Biochemistry, University of Delaware, Newark, DE 19716, United States

Corresponding Author

*edb@udel.edu. 


\section{List of Contents}

Detailed Experimental Procedures

S3

Correlation of Low-Pressure Isotherms to Surface Areas $\quad S 12$

Correlation of Bulk and Crystallographic Densities

S12

Nitrogen Adsorption Isotherms

S14

Methane Adsorption Isotherms

S28

References 


\section{Detailed Experimental Procedures}

General Considerations. All reagents were obtained from commercial vendors and used without purification. Dry N,N-Dimethylformamide (DMF) was obtained from a solvent purification system (SPS) and stored in a brown amber bottle under $4 \AA$ sieves. Low-pressure $\mathrm{N}_{2}$ adsorption measurements were obtained on a Micromeritics Tristar II PLUS at $77 \mathrm{~K}$. Low-pressure $\mathrm{CO}_{2}$ adsorption measurements were obtained on a Micromeritics Tristar 3000 at $195 \mathrm{~K}$. High-pressure isotherms were measured with a PCTPro-2000 Volumetric adsorption Analyzer.

\section{Synthesis of Terphenyl-3,3",5,5"-tetracarboxylic acid (H H $_{4}$ tptc)}

1,4-Benzenediboronic acid bis(pinacol) ester $(4.710 \mathrm{~g}, 14.27 \mathrm{mmol})$ and dimethyl-5bromoisophthalate $(8.432,30.88)$ were added to a 3-neck round bottom flask and dissolved in $300 \mathrm{~mL}$ of THF. Potassium carbonate $(9.061,65.56 \mathrm{mmol})$ was dissolved in $50 \mathrm{~mL}$ of water and the solution was added to the flask. A reflux condenser was attached and the vessel was placed under nitrogen for 30 minutes before $\mathrm{Pd}(\mathrm{DPPF}) \mathrm{Cl}_{2}(0.520 \mathrm{~g}, 0.7107 \mathrm{mmol})$ was added and the vessel was sealed under nitrogen and heated to $80^{\circ} \mathrm{C}$ overnight. The THF was removed in vacuo, additional water was added with sodium hydroxide and the reaction was heated to $100{ }^{\circ} \mathrm{C}$ overnight. The solution was filtered over celite and the filtrate was slowly acidified with $\mathrm{HCl}$ to precipitate the product. The precipitate was filtered off and dried yielding $3.086 \mathrm{~g}(71.5 \%)$ of $\mathrm{H}_{4}$ tptc. ${ }^{1} \mathrm{H}$ NMR (400 MHz, DMSO) $\delta 8.49$ (s, 3H), 8.44 (s, 4H), 7.90 (s, 4H).

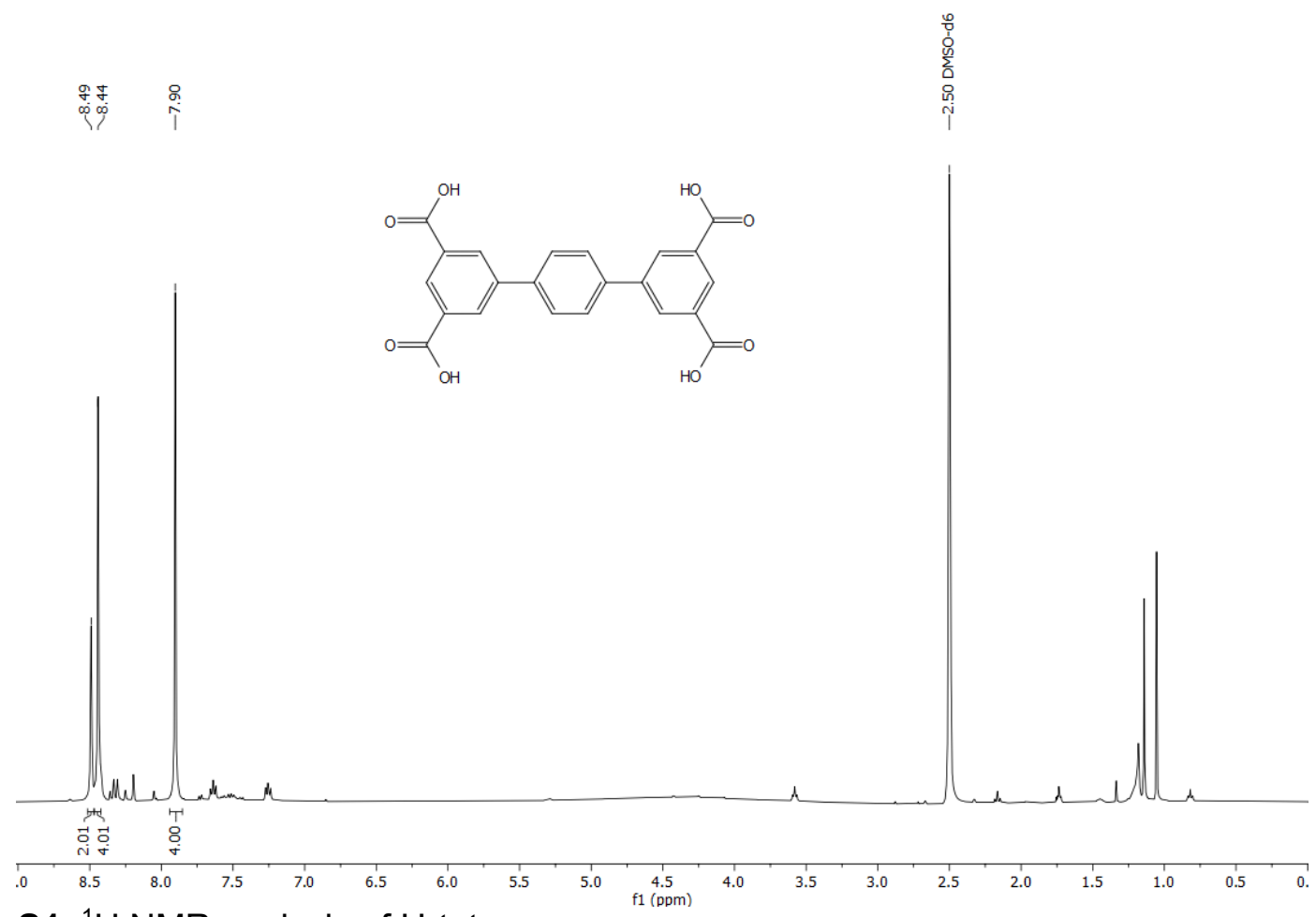

Figure S1. ${ }^{1} \mathrm{H}$ NMR analysis of $\mathrm{H}_{4}$ tptc.

\section{Synthesis of Quaterphenyl-3,3"',5,5"'-tetracarboxylic acid $\left(\mathrm{H}_{4} \mathrm{qptc}\right)$}

4,4'-diiodobiphenyl (3.495 g, $8.608 \mathrm{mmol}$ ) and 5-bis(methoxycarbonyl)benzeneboronic acid pinacol ester $(6.026 \mathrm{~g}, 18.83 \mathrm{mmol})$ were added to a 3-neck round bottom flask and dissolved in $300 \mathrm{~mL}$ of THF. Potassium carbonate $(11.137 \mathrm{~g}, 80.59 \mathrm{mmol})$ was dissolved in $50 \mathrm{~mL}$ of water and the solution was added to the flask. A reflux condenser was attached and the vessel was placed under nitrogen for 30 minutes before $\mathrm{Pd}(\mathrm{DPPF}) \mathrm{Cl}_{2}(0.466 \mathrm{~g}, 0.6369 \mathrm{mmol})$ was added and 
the vessel was sealed under nitrogen and heated to $80^{\circ} \mathrm{C}$ overnight. The THF was removed in vacuo, additional water was added with sodium hydroxide and the reaction was heated to $100{ }^{\circ} \mathrm{C}$ overnight. The solution was filtered over celite and the filtrate was slowly acidified with $\mathrm{HCl}$ to precipitate the product. The precipitate was filtered off and dried yielding $2.140 \mathrm{~g}(53.6 \%)$ of $\mathrm{H}_{4}$ qptc. ${ }^{1} \mathrm{H}$ NMR (400 MHz, DMSO) $\delta 8.48$ (s, 2H), 8.45 (s, 4H), 7.90 (m, 8H).
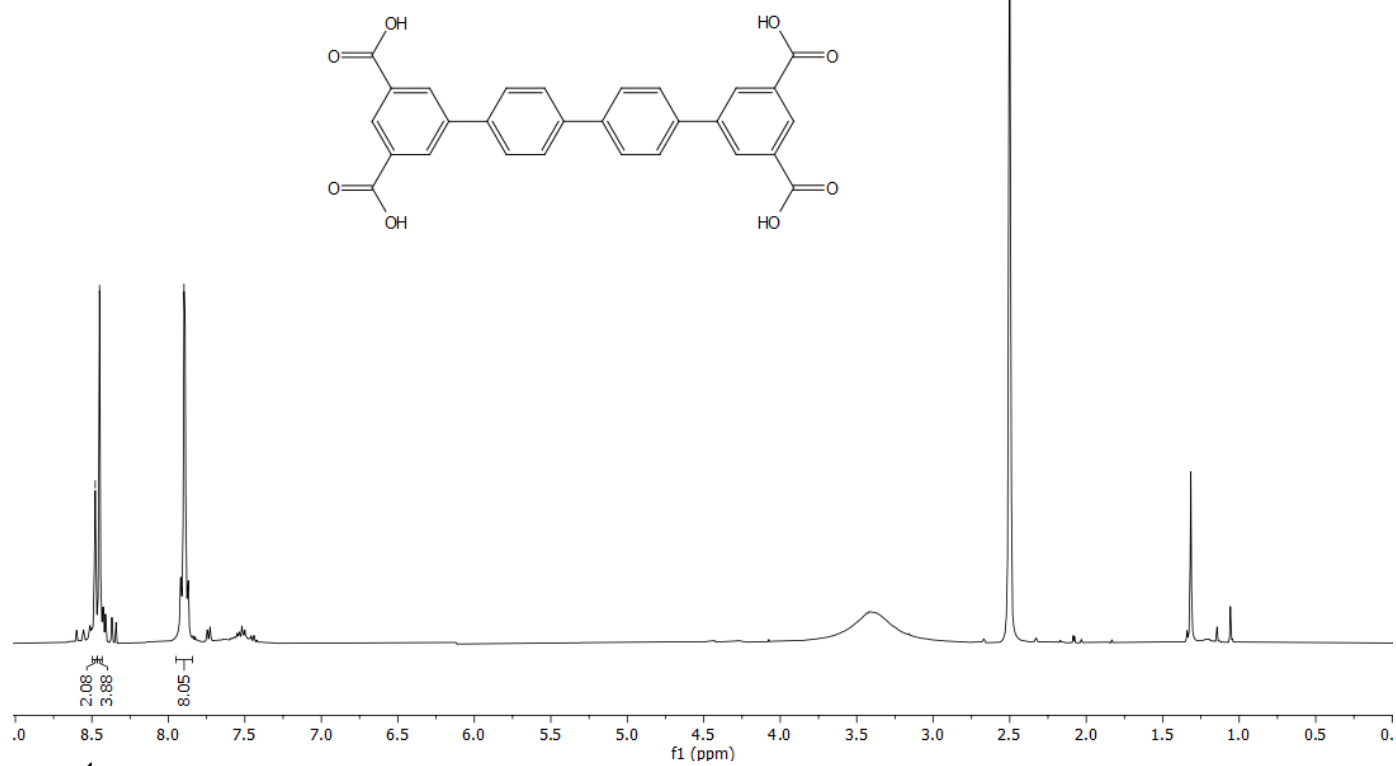

Figure S2. ${ }^{1} \mathrm{H}$ NMR analysis of $\mathrm{H}_{4} \mathrm{qptc}$.

Synthesis of 4,6-dihydroxyisophthalic acid $\left(\mathrm{H}_{4} \mathrm{~m}\right.$-dobdc)

$\mathrm{H}_{4}$ m-dobdc was prepared according to literature methods. ${ }^{1}\left(400 \mathrm{MHz}, \mathrm{DMSO}-\mathrm{d}_{6}\right) \delta 8.28(\mathrm{~s}, 1 \mathrm{H})$, $6.35(\mathrm{~s}, 1 \mathrm{H})$. 


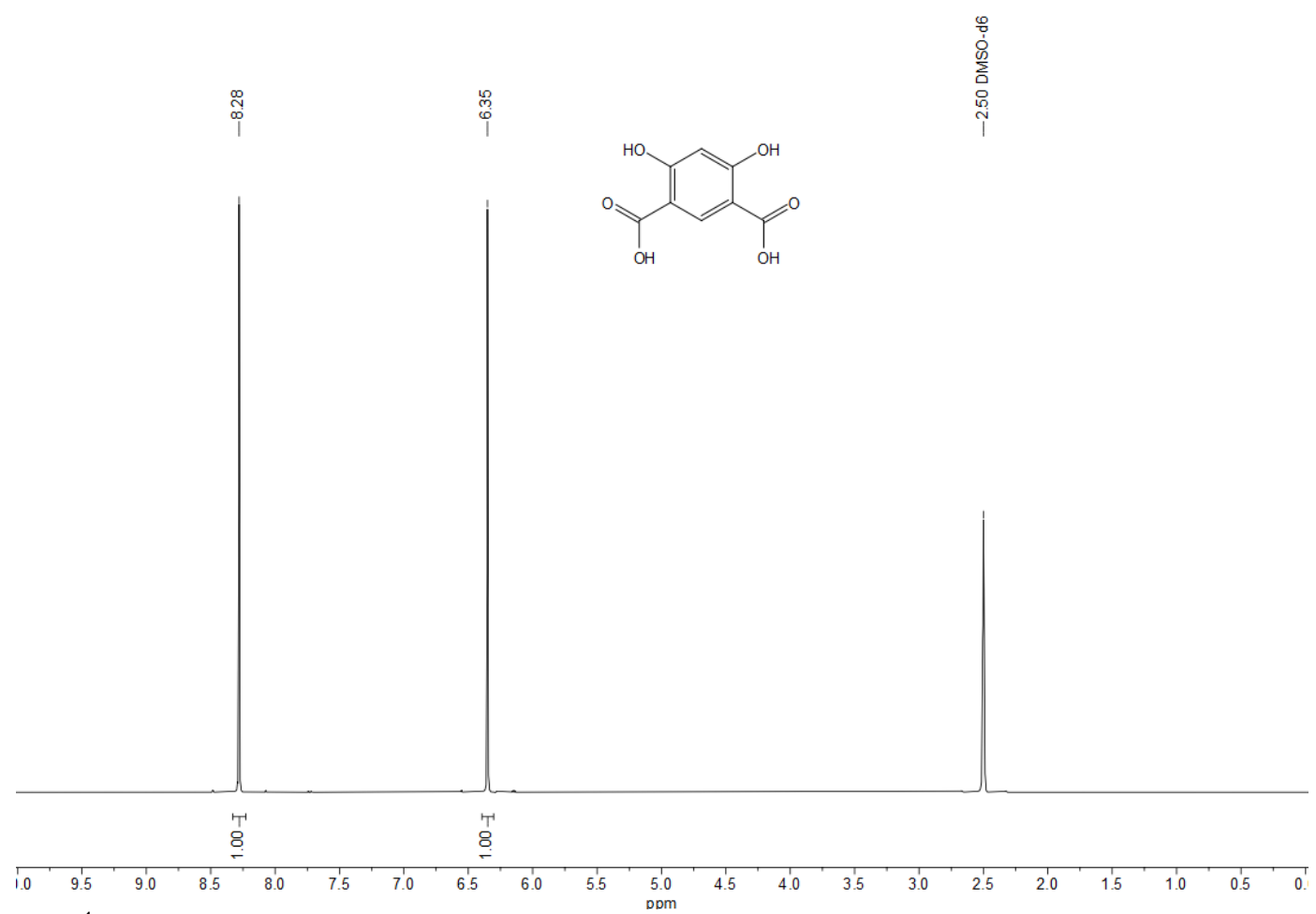

Figure S3. ${ }^{1} \mathrm{H}$ NMR analysis of $\mathrm{H}_{4} m$-dobdc. 
Synthesis of 1,3,5-Tris(4-carboxyphenyl)benzene $\left(\mathrm{H}_{3} \mathrm{BTB}\right)$

BTB was prepared according to literature methods. ${ }^{2}\left(400 \mathrm{MHz}, \mathrm{DMSO}-\mathrm{d}_{6}\right) \delta 8.09(\mathrm{~s}, 3 \mathrm{H}), 8.06(\mathrm{~s}$, $12 \mathrm{H})$.

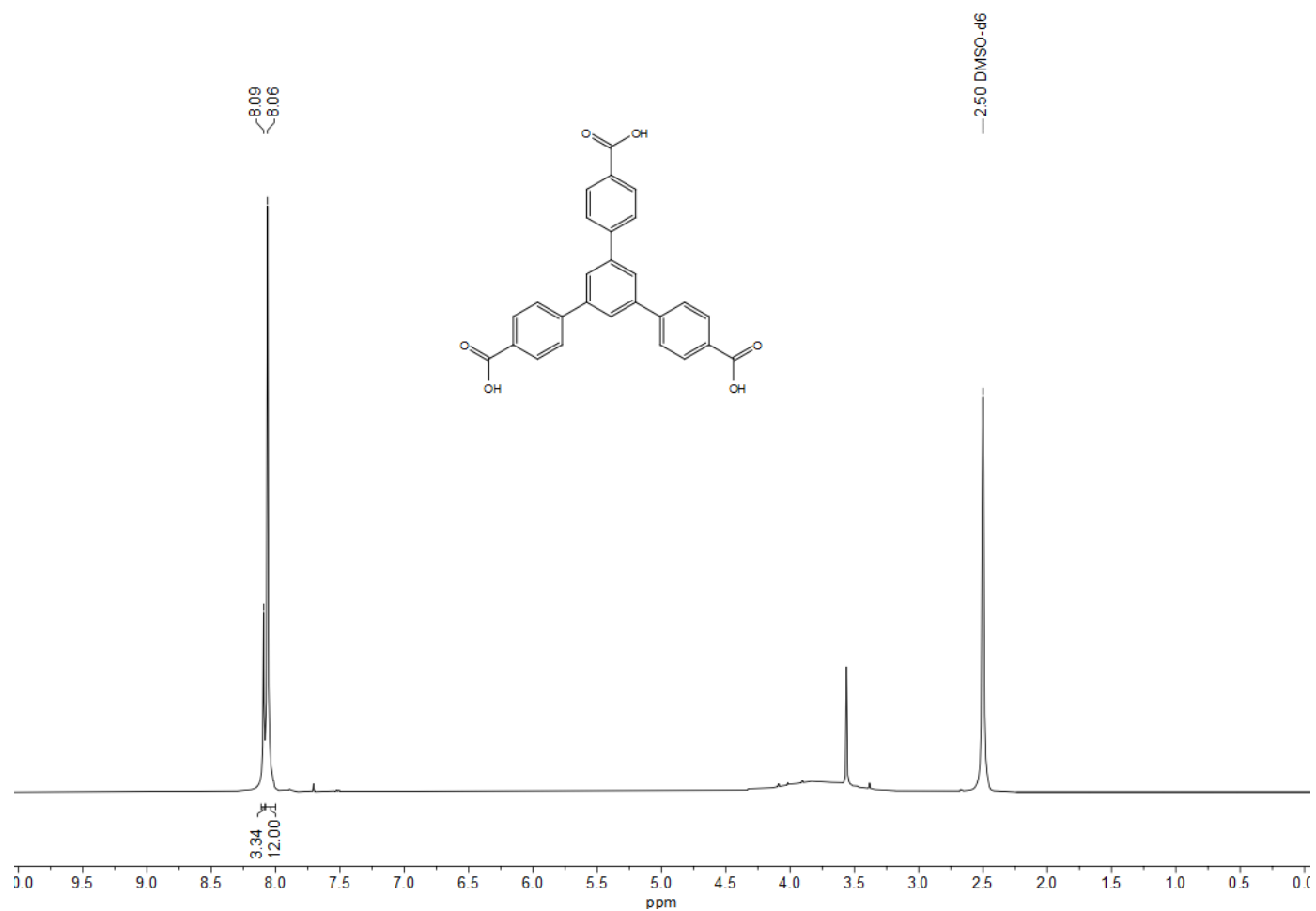

Figure S4. ${ }^{1} \mathrm{H}$ NMR analysis of $\mathrm{H}_{3} \mathrm{BTB}$. 


\section{Synthesis of 5,5'-(9,10-Anthracenediyl)bis(1,3-benzenedimethoxycarbonyl) $\left(\left(\mathrm{CH}_{3}\right)_{4}\right.$ adip)}

The following synthesis has been slightly modified from a previously published paper. ${ }^{3} 9,10-$ Dibromoanthracene (2 g, $8 \mathrm{mmol})$, dimethyl 5-(4,4,5,5-tetramethyl-1,3,2-dioxaborolan-2-yl) isophthalate $(4.2 \mathrm{~g}, 13.1 \mathrm{mmol})$, cesium fluoride $(5.47 \mathrm{~g}, 36 \mathrm{mmol})$, and [1,1'Bis(diphenylphosphino)ferrocene]dichloropalladium(II) ( $\left.\mathrm{Pd}(\mathrm{dppf}) \mathrm{Cl}_{2}\right)(221 \mathrm{mg}, 0.302 \mathrm{mmol}$ ) were added to a three-neck round-bottom flask under an $\mathrm{N}_{2}$ atmosphere. A mixture of dioxane $(42 \mathrm{~mL})$ and $\mathrm{H}_{2} \mathrm{O}(21 \mathrm{~mL})$ was sparged with $\mathrm{N}_{2}$ for 1 hour before being added to the reaction flask via cannula transfer. The mixture was heated at $100{ }^{\circ} \mathrm{C}$ for 4 days under positive $\mathrm{N}_{2}$ pressure. After the reaction was completed, the mixture was cooled to room temperature and the precipitate was collected via vacuum filtration. The precipitate was dried in an oven at $120^{\circ} \mathrm{C}$ for 24 hours to yield $\left(\mathrm{CH}_{3}\right)_{4}$ adip $(4.2 \mathrm{~g})$.

\section{Synthesis of 5,5-(9,10-Anthracenediyl)bis(1,3-benzenedicarboxylic acid) $\left(\mathrm{H}_{4}\right.$ adip)}

The following synthesis has been modified from a previously published paper. ${ }^{3}\left(\mathrm{CH}_{3}\right)_{2}$ adip $(4.2 \mathrm{~g}$, $7 \mathrm{mmol})$ and $\mathrm{NaOH}(3.67 \mathrm{~g}, 91.8 \mathrm{mmol})$ were added to a mixture of tetrahydrofuran $(40 \mathrm{~mL})$, ethanol $(40 \mathrm{~mL})$, and $\mathrm{H}_{2} \mathrm{O}(60 \mathrm{~mL})$ and heated at $60^{\circ} \mathrm{C}$ overnight. The solution was allowed to cool to room temperature before excess solvent was removed via rotary evaporation. The solution was acidified using concentrated $\mathrm{HCl}$ and the resulting precipitate was collected via vacuum filtration. (400MHz, DMSO-d $\left.\mathrm{d}_{6}\right) \delta 8.72(\mathrm{t}, 2 \mathrm{H}), 8.20(\mathrm{~d}, 4 \mathrm{H}), 7.54(\mathrm{~m}, 4 \mathrm{H}), 7.54(\mathrm{~m}, 4 \mathrm{H})$.
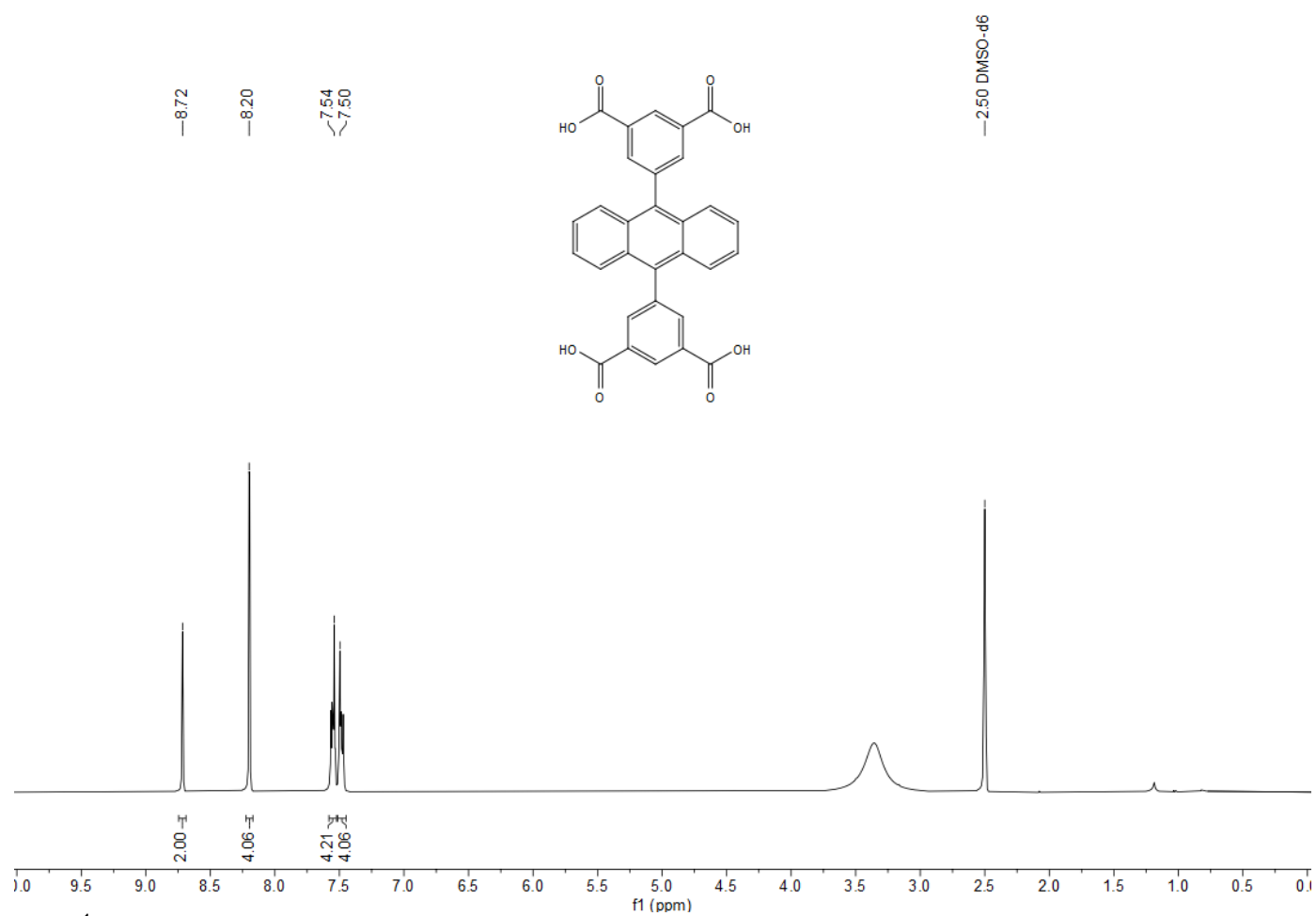

Figure S5. ${ }^{1} \mathrm{H}$ NMR analysis of $\mathrm{H}_{4}$ adip. 


\section{Synthesis of AI-MIL-53}

The following synthesis has been modified from a previously published paper. ${ }^{4} \mathrm{Al}\left(\mathrm{NO}_{3}\right)_{3} \cdot 9 \mathrm{H}_{2} \mathrm{O}$ $(3.90 \mathrm{~g}, 18.3 \mathrm{mmol})$, terephthalic acid $(0.864 \mathrm{~g}, 5.2 \mathrm{mmol})$, and $\mathrm{H}_{2} \mathrm{O}(15 \mathrm{~mL})$ were placed in a 25 $\mathrm{mL}$ Teflon-lined steel autoclave and heated at $220^{\circ} \mathrm{C}$ for 72 hours. The white powder was collected and washed three times with DMF at $65^{\circ} \mathrm{C}$ and three times with methanol. The material was then dried by vacuum activation at $300{ }^{\circ} \mathrm{C}$ for 12 hours.

\section{Synthesis of CC3-R}

The following synthesis has been prepared according to literature methods. ${ }^{5}$

\section{Synthesis of $\mathrm{Co}_{2}$ (m-dobdc)}

The following synthesis has been modified from a previously published paper. ${ }^{1}$ Anhydrous methanol $(70 \mathrm{~mL})$ and anhydrous DMF $(130 \mathrm{~mL})$ were sparged with $\mathrm{N}_{2}$ for one hour in a $500 \mathrm{~mL}$ three-neck round-bottom flask equipped with a reflux condenser. $\mathrm{CoCl}_{2}(1.05 \mathrm{~g}, 8.08 \mathrm{mmol})$ and $\mathrm{H}_{4} m$-dobdc $(0.64 \mathrm{~g}, 3.23 \mathrm{mmol})$ were added under positive $\mathrm{N}_{2}$ pressure and stirred at $120^{\circ} \mathrm{C}$ for 18 hours. The solid was isolated and washed with DMF at $70^{\circ} \mathrm{C}$ for 24 hours, followed by four more washes with methanol at $70^{\circ} \mathrm{C}$. The material was then activated under dynamic vacuum at $180^{\circ} \mathrm{C}$ for 24 hours.

\section{Synthesis of $\mathrm{Co}_{2}(\mathrm{bdc})_{2} \mathrm{dabco}$}

The following synthesis has been modified from a previously published paper. ${ }^{6} \mathrm{Co}\left(\mathrm{NO}_{3}\right)_{2} \cdot 6 \mathrm{H}_{2} \mathrm{O}$ $(0.367 \mathrm{~g}, 1.26 \mathrm{mmol})$, terephthalic acid $(0.209 \mathrm{~g}, 1.26 \mathrm{mmol}), 1,4$-diazobycyclo[2.2.2]octane $(0.070 \mathrm{~g}, 0.624 \mathrm{mmol})$, and DMF $(15 \mathrm{~mL})$ were added to a $25 \mathrm{~mL}$ Teflon-lined steel autoclave and heated at $110^{\circ} \mathrm{C}$ for 24 hours. The purple solid was collected then washed three times with DMF and three times with $\mathrm{CHCl}_{3}$, then activated under dynamic vacuum at $120^{\circ} \mathrm{C}$ for 24 hours.

\section{Synthesis of Co-MOF-74}

The following synthesis has been modified from a previously published paper. ${ }^{7} \mathrm{Co}\left(\mathrm{NO}_{3}\right)_{2} \cdot 6 \mathrm{H}_{2} \mathrm{O}$ $(4.754 \mathrm{~g}, 16.3 \mathrm{mmol})$ and 2,5-dihydroxyterephthalic acid $(0.964 \mathrm{~g}, 4.87 \mathrm{mmol})$ were combined with a 1:1:1 (v/v/v) solution of DMF/ethanol/ $\mathrm{H}_{2} \mathrm{O}(400 \mathrm{~mL})$ in a $500 \mathrm{~mL}$ jar. The solution was sparged with $\mathrm{N}_{2}$ for one hour then placed in a $100^{\circ} \mathrm{C}$ oven for 24 hours. The solid was isolated and rinsed repeatedly with DMF, soaked three times in DMF at $120^{\circ} \mathrm{C}$ for 6 hours each, then soaked three more times in methanol at $60^{\circ} \mathrm{C}$ for 6 hours each. The material was collected then activated with flowing $\mathrm{N}_{2}$ at $180^{\circ} \mathrm{C}$ for 48 hours followed by dynamic vacuum at $180{ }^{\circ} \mathrm{C}$ for 24 hours.

\section{Synthesis of Cr-MIL-100}

The following synthesis has been modified from a previously published paper. ${ }^{8} \mathrm{CrO}_{3}(0.25 \mathrm{~g}, 2.5$ $\mathrm{mmol})$, trimesic acid $(0.525 \mathrm{~g}, 2.5 \mathrm{mmol})$, hydrofluoric acid $(48 \%, 0.091 \mathrm{~mL})$, and $\mathrm{DI} \mathrm{H}_{2} \mathrm{O}(12 \mathrm{~mL})$ were combined in a $25 \mathrm{~mL}$ Teflon-lined steel autoclave, then heated at $220^{\circ} \mathrm{C}$ for 48 hours. The solid was collected, washed with $\mathrm{H}_{2} \mathrm{O}$ then acetone, followed by activation with flowing $\mathrm{N}_{2}$ at 100 ${ }^{\circ} \mathrm{C}$ for 24 hours.

\section{Synthesis of $\mathrm{Cr}_{24}(\mathrm{bdc}-\mathrm{tBu})_{24}$}

The following synthesis has been prepared according to literature methods. ${ }^{9}$ 


\section{Synthesis of $\mathrm{Cu}_{2}$ (adip) (PCN-14)}

The following synthesis has been modified from a previously published paper. ${ }^{3} \mathrm{Cu}\left(\mathrm{NO}_{3}\right)_{2} \cdot 2.5 \mathrm{H}_{2} \mathrm{O}$ $(2.4 \mathrm{~g}, 10.3 \mathrm{mmol})$ and $\mathrm{H}_{4}$ adip $(0.6 \mathrm{~g}, 1.2 \mathrm{mmol})$ were solvated in DMF $(180 \mathrm{~mL})$. To the solution, 10 drops of $\mathrm{HBF}_{4}$ were added before being distributed to $20 \mathrm{~mL}$ scintillation vials $(20 \mathrm{x})$. The vials were heated at $75{ }^{\circ} \mathrm{C}$ for 24 hours then were cooled to room temperature. The green material was washed with fresh DMF three times at 24 hour intervals followed by solvent exchange with methanol three times at 24 hour intervals. The material was activated with flowing $\mathrm{N}_{2}$ at $140{ }^{\circ} \mathrm{C}$ for 24 hours.

\section{Synthesis of DUT-8 (Zn)}

The following synthesis has been modified from a previously published paper. ${ }^{10}$ The following reactants were dissolved separately: $\mathrm{Zn}\left(\mathrm{NO}_{3}\right)_{2} \cdot 6 \mathrm{H}_{2} \mathrm{O}(0.312 \mathrm{~g}, 1.05 \mathrm{mmol})$ in DMF $(5 \mathrm{~mL}), 2,6-$ naphthalenedicarboxylic acid $(0.227 \mathrm{~g}, 1.05 \mathrm{mmol})$ in DMF $(15 \mathrm{~mL})$, and 1,4diazobycyclo[2.2.2] octane $(0.059 \mathrm{~g}, 0.53 \mathrm{mmol})$ in DMF $(5 \mathrm{~mL})$. The mixtures were then combined and sonicated for 10 minutes before being transferred to a Teflon vial and heated at $120{ }^{\circ} \mathrm{C}$ for 48 hours. The material was then collected and washed three times with DMF, followed by three washes with methanol before activation under dynamic vacuum at $100{ }^{\circ} \mathrm{C}$ for 24 hours.

\section{Synthesis of $\mathrm{Fe}_{24}(\mathrm{Me}-\mathrm{bdc})_{24}(\mathrm{dabco})_{6}$}

The following synthesis has been prepared according to literature methods. ${ }^{11}$

\section{Synthesis of HKUST-1}

The following synthesis has been modified from a previously published paper. ${ }^{12} \mathrm{Cu}\left(\mathrm{NO}_{3}\right)_{2} \cdot 2.5 \mathrm{H}_{2} \mathrm{O}$ $(3.87 \mathrm{~g}, 15.3 \mathrm{mmol})$, trimesic acid $(1.93 \mathrm{~g}, 9.18 \mathrm{mmol})$, and DMF $(100 \mathrm{~mL})$ were added to a 250 $\mathrm{mL}$ round-bottom flask then stirred at $75{ }^{\circ} \mathrm{C}$ for 48 hours. The solid was collected then soaked three times with DMF at $75^{\circ} \mathrm{C}$ for 12 hours then washed three more times with methanol at room temperature for 12 hours. The material was activated under vacuum at $180^{\circ} \mathrm{C}$ for 60 hours.

\section{Synthesis of $\mathrm{MO}_{24}\left(\mathrm{bdc}-\mathrm{NH}_{2}\right)_{24}$}

The following synthesis has been modified from a previously published paper. ${ }^{13} \mathrm{Mo}_{2}(\mathrm{OAc})_{4}(0.100$ $\mathrm{g}, 0.234 \mathrm{mmol})$ and 5-amino-1,3-benzenedicarboxylic acid $(0.0423 \mathrm{~g}, 0.234 \mathrm{mmol})$ were added to a $20 \mathrm{~mL}$ scintillation vial and dissolved in $10 \mathrm{~mL}$ of anhydrous $\mathrm{N}, \mathrm{N}$-Diethylformamide (DEF). The solution was heated at $120^{\circ} \mathrm{C}$ for 2 days. The orange crystalline powder was washed at room temperature three times with anhydrous THF, exchanging the solvent every 8 hours, and dried under vacuum to yield a dark orange powder.

\section{Synthesis of $\mathrm{Mo}_{24}(\mathrm{bdc}-\mathrm{OH})_{24}$}

The following synthesis has been modified from a previously published paper. ${ }^{13} \mathrm{Mo}_{2}(\mathrm{OAc})_{4}(0.100$ $\mathrm{g}, 0.234 \mathrm{mmol})$ and 5-hydroxy-1,3-benzenedicarboxylic acid $(0.0426 \mathrm{~g}, 0.234 \mathrm{mmol})$ were added to a $20 \mathrm{~mL}$ scintillation vial and dissolved in $10 \mathrm{~mL}$ of anhydrous N,N'-Dimethylpropyleneurea (DMPU). The solution was heated at $120^{\circ} \mathrm{C}$ for 2 days. The bright red crystals were washed at room temperature three times with anhydrous THF, exchanging the solvent every 8 hours, and dried under vacuum to yield a bright orange powder.

\section{Synthesis of $\mathrm{Mo}_{24}(\mathrm{bdc}-\mathrm{tBu})_{24}$}

The following synthesis was prepared as previously published. ${ }^{11}$ In an $\mathrm{N}_{2}$ glovebox, $\mathrm{Mo}_{2}(\mathrm{OAc})_{4}$ $(0.10 \mathrm{~g}, 0.234 \mathrm{mmol})$ and tert-butylisophthalic acid $(0.052 \mathrm{~g}, 0.234 \mathrm{mmol})$ were dissolved in 10 $\mathrm{mL}$ of $\mathrm{N}, \mathrm{N}^{\prime}$-dimethylpropyleneurea (DMPU) with 5 drops of pyridine and heated at $110^{\circ} \mathrm{C}$ for 1 day to yield large red crystals. They crystals were soaked in $20 \mathrm{~mL}$ of anhydrous, air-free methanol for $48 \mathrm{~h}$, with the solvent replaced by fresh methanol every $8 \mathrm{~h}$. The material was isolated by vacuum filtration and were activated under dynamic vacuum at $25^{\circ} \mathrm{C}$. 


\section{Synthesis of MOF-177}

The following synthesis has been modified from a previously published paper. ${ }^{14} \mathrm{Zn}\left(\mathrm{NO}_{3}\right)_{2} \cdot 6 \mathrm{H}_{2} \mathrm{O}$ (3.6 g, $12.1 \mathrm{mmol})$ and $\mathrm{H}_{3} \mathrm{BTB}(0.72 \mathrm{~g}, 1.64 \mathrm{mmol})$ were dissolved in DMF $(240 \mathrm{~mL})$ then distributed to $20 \mathrm{~mL}$ scintillation vials $(20 \mathrm{x})$. The vials were capped with Teflon-lined caps then heated at $70^{\circ} \mathrm{C}$ for 7 days. The crystals were collected, washed three times with DMF, then three times with chloroform at $65{ }^{\circ} \mathrm{C}$ for 24 hours each. The material was activated at $100{ }^{\circ} \mathrm{C}$ under dynamic vacuum for 24 hours.

\section{Synthesis of $\mathrm{Ni}(\mathrm{ndc})(\mathrm{ted})_{0.5}$}

The following synthesis has been modified from a previously published paper. ${ }^{15} \mathrm{Ni}\left(\mathrm{NO}_{3}\right)_{2} \cdot 6 \mathrm{H}_{2} \mathrm{O}$ $(0.136 \mathrm{~g}, 0.47 \mathrm{mmol}), 2,6$-naphthalenedicarboxylic acid $(0.092 \mathrm{~g}, 0.43 \mathrm{mmol})$ in $15 \mathrm{~mL} \mathrm{DMF}$, and 1,4-diazobycyclo[2.2.2]octane $(0.105 \mathrm{~g}, 0.94 \mathrm{mmol})$ were combined and dissolved in $15 \mathrm{~mL}$ DMF. The mixture was sonicated before being transferred to a Teflon lined Parr bomb and heated at $135^{\circ} \mathrm{C}$ for 3 days. The material was collected, washed three times with DMF, then activated with flowing $\mathrm{N}_{2}$ at $120^{\circ} \mathrm{C}$ for 24 hours.

\section{Synthesis of $\mathrm{Ni}_{2}$ (m-dobdc)}

The following synthesis has been modified from a previously published paper. ${ }^{1}$ Anhydrous methanol $(70 \mathrm{~mL})$ and anhydrous DMF $(130 \mathrm{~mL})$ were added to a $500 \mathrm{~mL}$ three-neck roundbottom flask equipped with a reflux condenser and sparged with $\mathrm{N}_{2}$ for one hour. $\mathrm{NiCl}_{2}(1.05 \mathrm{~g}$, $8.02 \mathrm{mmol})$ and $\mathrm{H}_{4}\left(\mathrm{~m}\right.$-dobdc) $(0.640 \mathrm{~g}, 3.23 \mathrm{mmol})$ were added under $\mathrm{N}_{2}$ pressure and stirred at $120^{\circ} \mathrm{C}$ for 18 hours. The solid was isolated and washed with DMF at $70^{\circ} \mathrm{C}$ for 24 hours, followed by four more washes with methanol at $70{ }^{\circ} \mathrm{C}$. The material was then activated under dynamic vacuum at $180^{\circ} \mathrm{C}$ for 24 hours.

\section{Synthesis of Ni-MOF-74}

The following synthesis has been modified from a previously published paper. ${ }^{7} \mathrm{Ni}\left(\mathrm{NO}_{3}\right)_{2} \cdot 6 \mathrm{H}_{2} \mathrm{O}$ (5.23 g, $18 \mathrm{mmol})$ and $\mathrm{H}_{4}$ (dobdc) $(1.42 \mathrm{~g}, 7.2 \mathrm{mmol}$ ) were combined with anhydrous DMF (350 $\mathrm{mL}$ ) and anhydrous methanol $(42 \mathrm{~mL})$ in a $500 \mathrm{~mL}$ Schlenk flask. The solution stirred under $\mathrm{N}_{2}$ pressure at $120^{\circ} \mathrm{C}$ for 18 hours. The solution was allowed to cool while the yellow powder settled to the bottom of the flask. The solution was removed via cannula and replaced with fresh DMF, then soaked at $100{ }^{\circ} \mathrm{C}$ for 6 hours. The solution was removed and replaced twice more with fresh DMF, then repeated three more times with fresh methanol at $60^{\circ} \mathrm{C}$ for 6 hours each. The material was collected then activated with flowing $\mathrm{N}_{2}$ at $180^{\circ} \mathrm{C}$ for 48 hours followed by dynamic vacuum at $180^{\circ} \mathrm{C}$ for 24 hours.

\section{Synthesis of NOTT-101}

NOTT-101 was prepared according to literature methods. ${ }^{16}$

\section{Synthesis of (sc4a) Fe(bdc)}

(sc4a)Fe(bdc) was prepared according to literature methods. ${ }^{17}$

\section{Synthesis of (sc4a) Fe(btc)}

(sc4a) $\mathrm{Fe}(\mathrm{btc})$ was prepared according to literature methods. ${ }^{17}$

\section{Synthesis of (sc4a) Fe(tatb)}

(sc4a)Fe(btc) was prepared according to literature methods. ${ }^{17}$

\section{Synthesis of Ti-MIL-101}

The following synthesis has been prepared according to literature methods. ${ }^{18}$ 


\section{Synthesis of Ti-MIL-125}

The following synthesis has been modified from a previously published paper. ${ }^{19}$ Dry DMF (25 $\mathrm{mL}$ ) was added to a $100 \mathrm{~mL}$ three-neck round-bottom flask fitted with a reflux condenser and sparged with $\mathrm{N}_{2}$ for 1 hour. Terephthalic acid $(1.925 \mathrm{~g}, 11.6 \mathrm{mmol})$ was added to the flask under positive $\mathrm{N}_{2}$ pressure, then stirred at $100{ }^{\circ} \mathrm{C}$ for 1 hour. Methanol $(7 \mathrm{~mL})$ was added to the flask and stirred for another hour at $100{ }^{\circ} \mathrm{C}$. Finally, titanium (IV) butoxide $(2.1 \mathrm{~mL}, 6.17 \mathrm{mmol})$ was added to the solution and refluxed at $100{ }^{\circ} \mathrm{C}$ for 72 hours. The material was collected, washed three times with DMF for 24 hour intervals, three more times with methanol for 24 hour intervals. The material was activated by heating at $100^{\circ} \mathrm{C}$ under dynamic vacuum for 14 hours then at $200^{\circ} \mathrm{C}$ under dynamic vacuum for 24 hours.

\section{Synthesis of UiO-66 Defect Free}

Defect free UiO-66 was synthesized following a previously published paper. ${ }^{20}$ In a $20 \mathrm{~mL}$ vial ZrCl 4 $(90.0 \mathrm{mg}, 0.386 \mathrm{mmol})$ and terephthalic acid $(96.2 \mathrm{mg}, 0.579 \mathrm{mmol})$ were dissolved in dry DMF $(15 \mathrm{~mL})$ and sonicated until clear. DI $\mathrm{H}_{2} \mathrm{O}(50 \mu \mathrm{L})$ was then added to the vial, and the resulting vial was heated at $110{ }^{\circ} \mathrm{C}$ for 24 hours. The resulting product was isolated via centrifugation and washed three times with DMF and three times with methanol. The sample was then activated with flowing $\mathrm{N}_{2}$ while heating at $100{ }^{\circ} \mathrm{C}$ overnight for gas adsorption analysis.

\section{Synthesis of UiO-66 High surface area}

The following synthesis has been modified from a previously published paper. ${ }^{21}$ In a 1 liter glass media bottle $\mathrm{ZrCl}_{4}(4.00 \mathrm{~g}, 17.2 \mathrm{mmol})$, concentrated $\mathrm{HCl}(32 \mathrm{~mL})$, and DMF $(160 \mathrm{~mL})$ were sonicated for 20 minutes until fully dissolved. Terephthalic acid (3.94 g, $23.7 \mathrm{mmol})$ and DMF (320 $\mathrm{mL}$ ) was added to the mixture and sonicated for an additional 20 minutes. After sonication the 1liter glass media bottle was heated at $80{ }^{\circ} \mathrm{C}$ overnight. The resulting product was isolated via centrifugation and washed five times with DMF and five times with methanol. Material was then dried with flowing $\mathrm{N}_{2}$ at room temperature followed by vacuum activation at $100{ }^{\circ} \mathrm{C}$ for two days.

\section{Synthesis of UiO-67}

The following synthesis has been modified from a previously published paper. ${ }^{21}$ In a 1 -liter glass media bottle $\mathrm{ZrCl}_{4}(2.14 \mathrm{~g}, 9.20 \mathrm{mmol})$, concentrated $\mathrm{HCl}(16 \mathrm{~mL})$, and DMF $(160 \mathrm{~mL})$ were sonicated for 20 minutes until fully dissolved. Biphenyl-4,4'-dicraboxylic acid $(2.80 \mathrm{~g}, 11.9 \mathrm{mmol})$ and DMF (320 mL) was added to the mixture and sonicated for an additional 20 minutes. After sonication the 1 liter glass media bottle was heated at $80^{\circ} \mathrm{C}$ overnight. The resulting product was isolated via centrifugation and washed five times with DMF and five times with methanol. Material was then dried with flowing $\mathrm{N}_{2}$ at room temperature followed by vacuum activation at $125{ }^{\circ} \mathrm{C}$ for two days.

\section{Synthesis of $\mathrm{Zn}(\mathrm{bdc})(\mathrm{ted})_{0.5}$}

The following synthesis has been modified from a previously published paper. ${ }^{22} \mathrm{Zn}\left(\mathrm{NO}_{3}\right)_{2} \cdot 6 \mathrm{H}_{2} \mathrm{O}$ $(0.156 \mathrm{~g}, 0.52 \mathrm{mmol})$, terephthalic acid $(0.102 \mathrm{~g}, 0.61 \mathrm{mmol})$, and 1,4-diazobycyclo[2.2.2]octane $(0.036 \mathrm{~g}, 0.32 \mathrm{mmol})$ were combined and dissolved in $15 \mathrm{~mL}$ DMF. The mixture was transferred to a Teflon vial and heated at $120^{\circ} \mathrm{C}$ for 48 hours. The material was collected and washed three times with DMF, three times with $\mathrm{MeOH}$, and activated under a flow of $\mathrm{N}_{2}$ at $100{ }^{\circ} \mathrm{C} 24$ hours.

\section{Synthesis of $\mathbf{Z} \mathbf{n}_{2}(\mathbf{b d c})_{2}$ dabco}

The following synthesis has been modified from a previously published paper. ${ }^{23} \mathrm{Zn}\left(\mathrm{NO}_{3}\right)_{2} \cdot 6 \mathrm{H}_{2} \mathrm{O}$ $(3.22 \mathrm{~g}, 10.9 \mathrm{mmol})$, terephthalic acid $(1.62 \mathrm{~g}, 9.75 \mathrm{mmol})$, and 1,4-diazobycyclo[2.2.2]octane $(0.560 \mathrm{~g}, 4.99 \mathrm{mmol})$, and DMF $(120 \mathrm{~mL})$ were combined a $250 \mathrm{~mL}$ Teflon vial and heated at 120 ${ }^{\circ} \mathrm{C}$ for 48 hours. The material was collected, washed three times with DMF, followed by three washes with chloroform, and then activated under dynamic vacuum at $120^{\circ} \mathrm{C}$ for 24 hours. 


\section{Correlation of Low-Pressure Isotherms to Surface Areas}

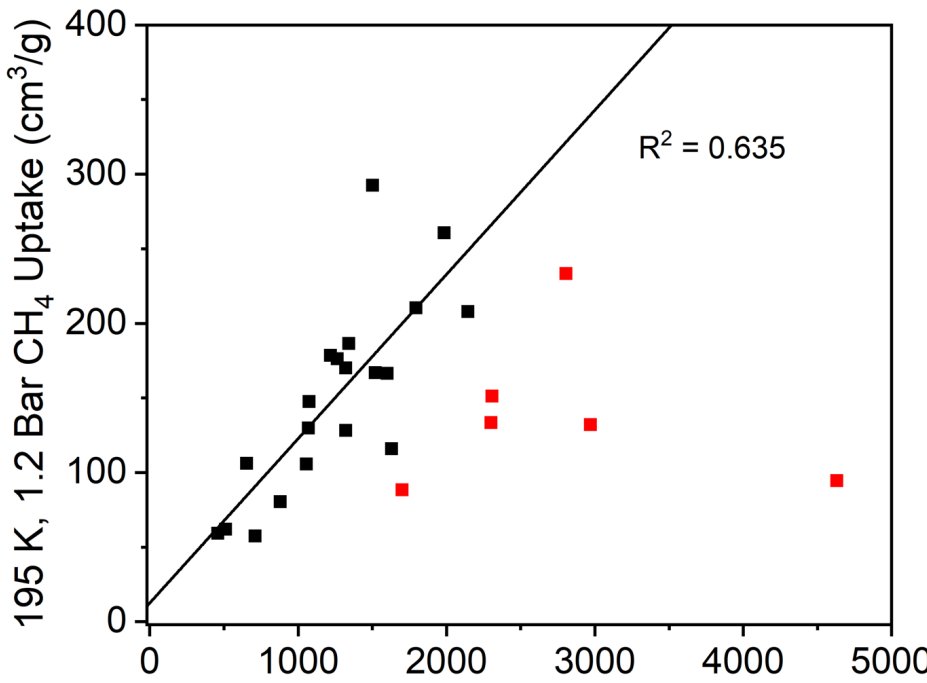

BET Surface Area $\left(\mathrm{m}^{2} / \mathrm{g}\right)$

Figure S6. Correlation between low-pressure methane capacities and $\mathrm{N}_{2}$ accessible BET surface areas.

\section{Correlation of Bulk and Crystallographic Densities}

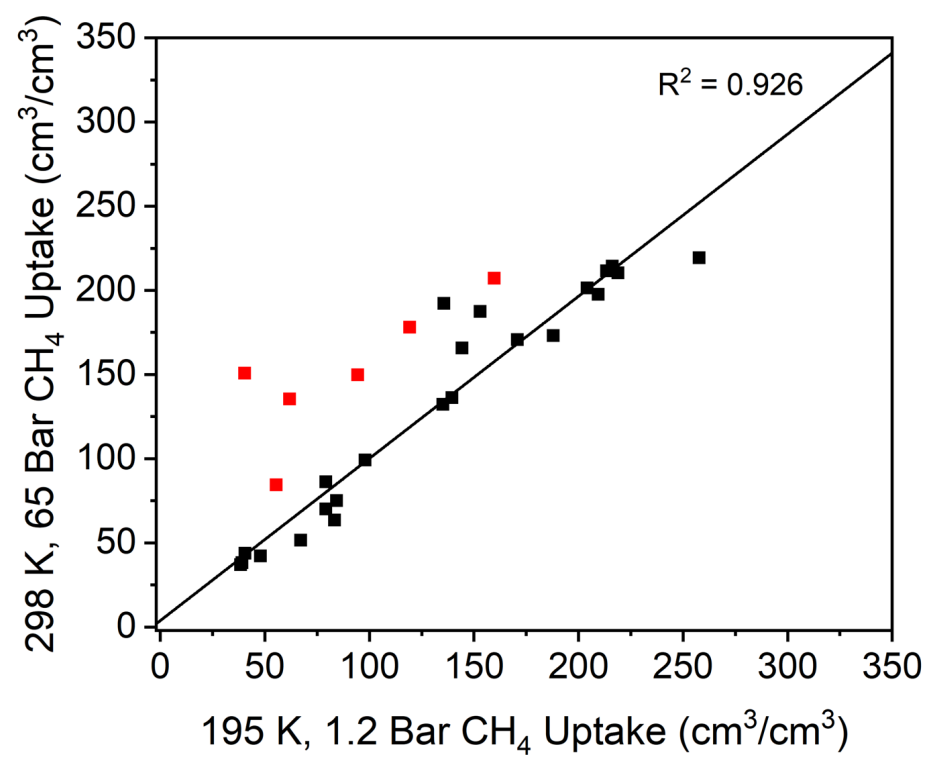

Figure S7. Correlation of volumetric capacities based on crystallographic densities. 
Table S1. Materials with their respective bulk and crystallographic densities.

\begin{tabular}{|c|c|c|}
\hline Material & $\begin{array}{l}\text { Crystallographic Density } \\
\qquad\left(\mathrm{g} / \mathrm{cm}^{3}\right)\end{array}$ & $\begin{array}{l}\text { Bulk Density } \\
\left(\mathrm{g} / \mathrm{cm}^{3}\right)\end{array}$ \\
\hline Al-MIL-53 & 0.978 & 0.281 \\
\hline CC3-R & 0.922 & 0.139 \\
\hline $\mathrm{Co}_{2}(m$-dobdc $)$ & 1.188 & 0.2559 \\
\hline $\mathrm{Co}_{2}(\mathrm{bdc})_{2}(\mathrm{dabco})$ & 0.815 & 0.2086 \\
\hline Co-MOF-74 & 1.173 & 0.777 \\
\hline Cr-MIL-100 & 0.7 & 0.187 \\
\hline $\mathrm{Cr}_{24}(\mathrm{tBu}-\mathrm{bdc})_{24}$ & 0.618 & 0.571 \\
\hline $\mathrm{Cu}_{2}$ (adip) $(\mathrm{PCN}-14)$ & 0.829 & 0.234 \\
\hline DUT-8 Zn & 0.68 & 0.449 \\
\hline HKUST-1 & 0.881 & 0.35 \\
\hline $\mathrm{Mo}_{24}(\mathrm{NH} 2-\mathrm{bdc})_{24}$ & 0.65 & 0.367 \\
\hline $\mathrm{Mo}_{24}\left({ }^{\mathrm{t}} \mathrm{Bu}-\mathrm{bdc}\right)_{24}$ & 0.676 & 0.61 \\
\hline MOF-177 & 0.427 & 0.25 \\
\hline $\mathrm{Ni}(\mathrm{ndc})$ ted $_{0.5}$ & 0.789 & 0.221 \\
\hline $\mathrm{Ni}_{2}(m-$ dobdc $)$ & 1.2 & 0.343 \\
\hline Ni-MOF-74 & 1.195 & 0.539 \\
\hline NOTT-101 & 0.684 & 0.139 \\
\hline (sc4a)Fe(bdc) & 0.773 & 0.6426 \\
\hline (sc4a)Fe(btc) & 0.895 & 0.776 \\
\hline (sc4a)Fe(tatb) & 0.836 & 0.5187 \\
\hline Ti-MIL-125 & 0.81 & 0.276 \\
\hline UiO-66 Defect Free & 1.32 & 0.268 \\
\hline UiO-66 High SA & 1.32 & 0.249 \\
\hline UiO-67 & 0.708 & 0.155 \\
\hline $\mathrm{Zn}(\mathrm{bdc})(\mathrm{ted})_{0.5}$ & 0.893 & 0.377 \\
\hline $\mathrm{Zn}_{2}(\mathrm{bdc})_{2} \mathrm{dabco}$ & 0.822 & 0.49 \\
\hline
\end{tabular}


Nitrogen Adsorption Isotherms

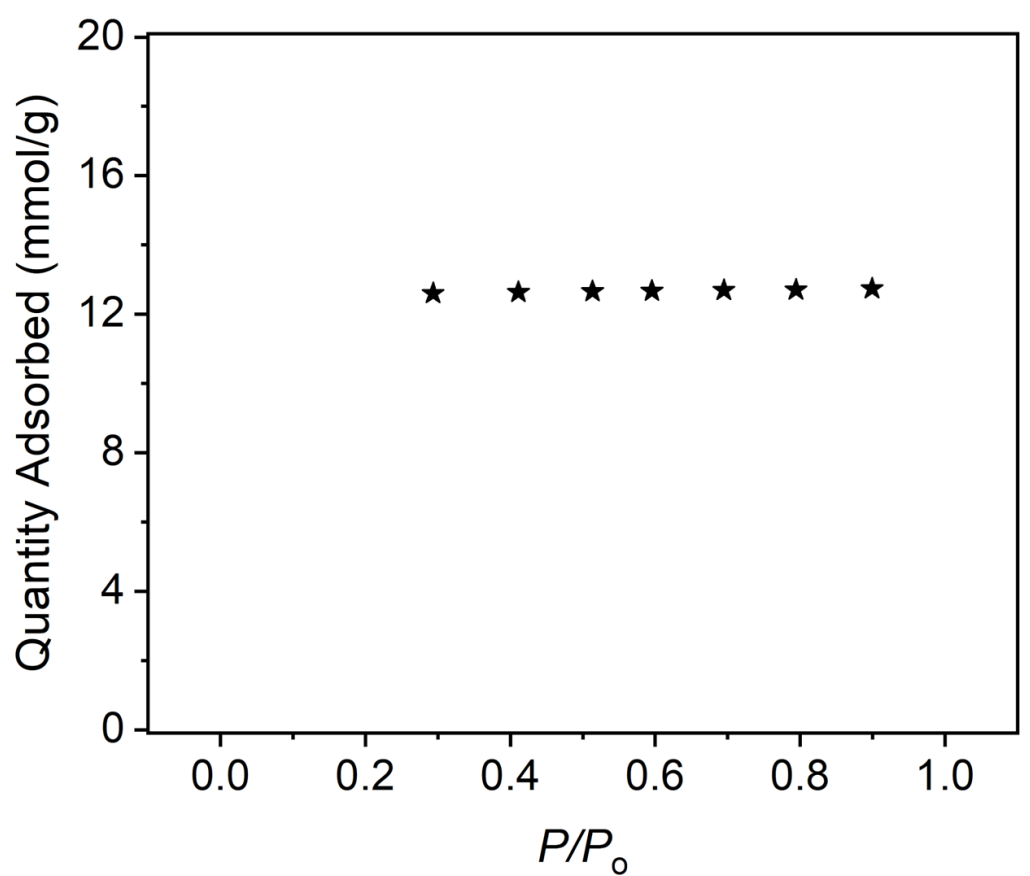

Figure S8. $\mathrm{N}_{2}$ adsorption isotherm at $77 \mathrm{~K}$ of Al-MIL-53.

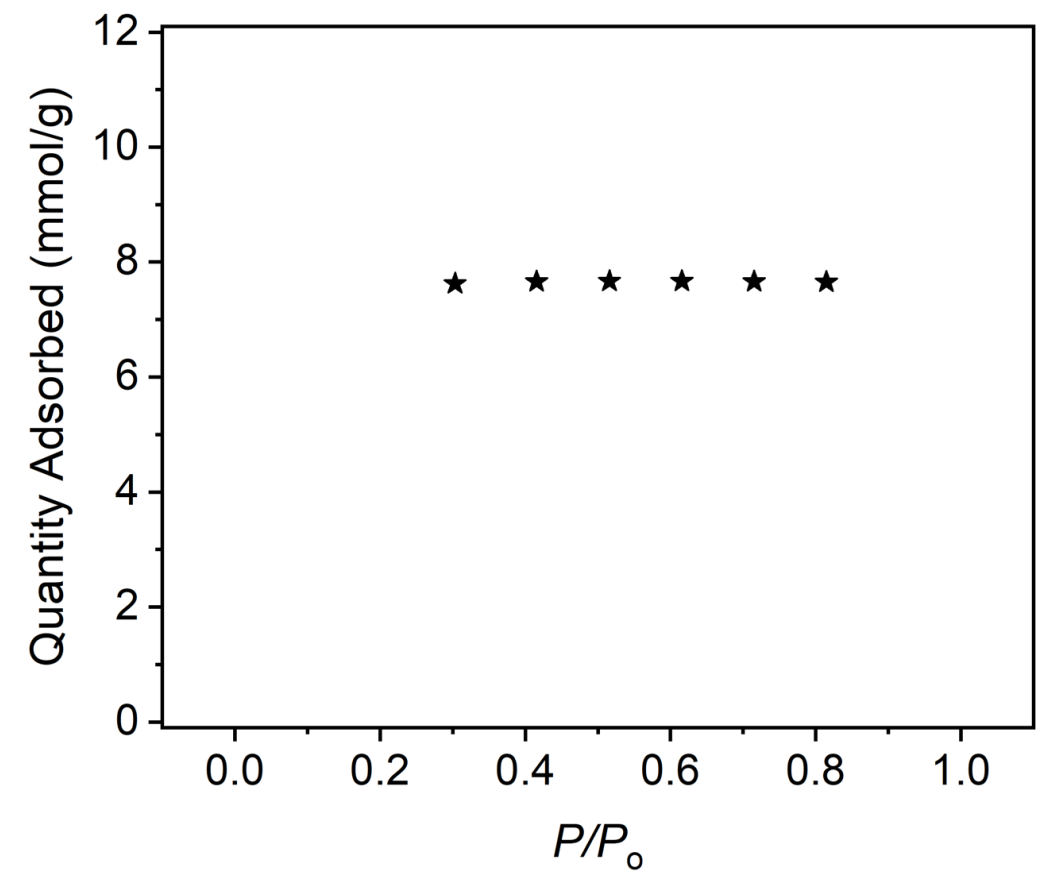

Figure S9. $\mathrm{N}_{2}$ adsorption isotherm at $77 \mathrm{~K}$ of CC3-R. 


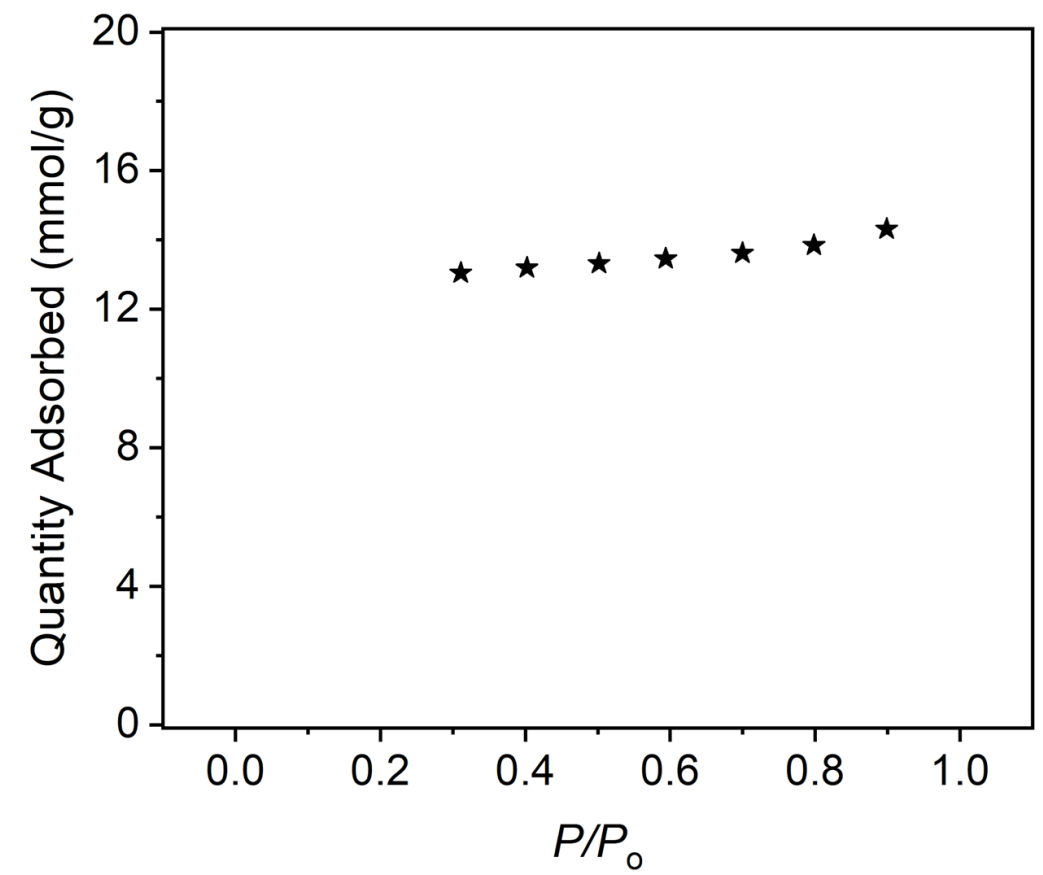

Figure S10. $\mathrm{N}_{2}$ adsorption isotherm at $77 \mathrm{~K}$ of $\mathrm{Co}_{2}(m$-dobdc).

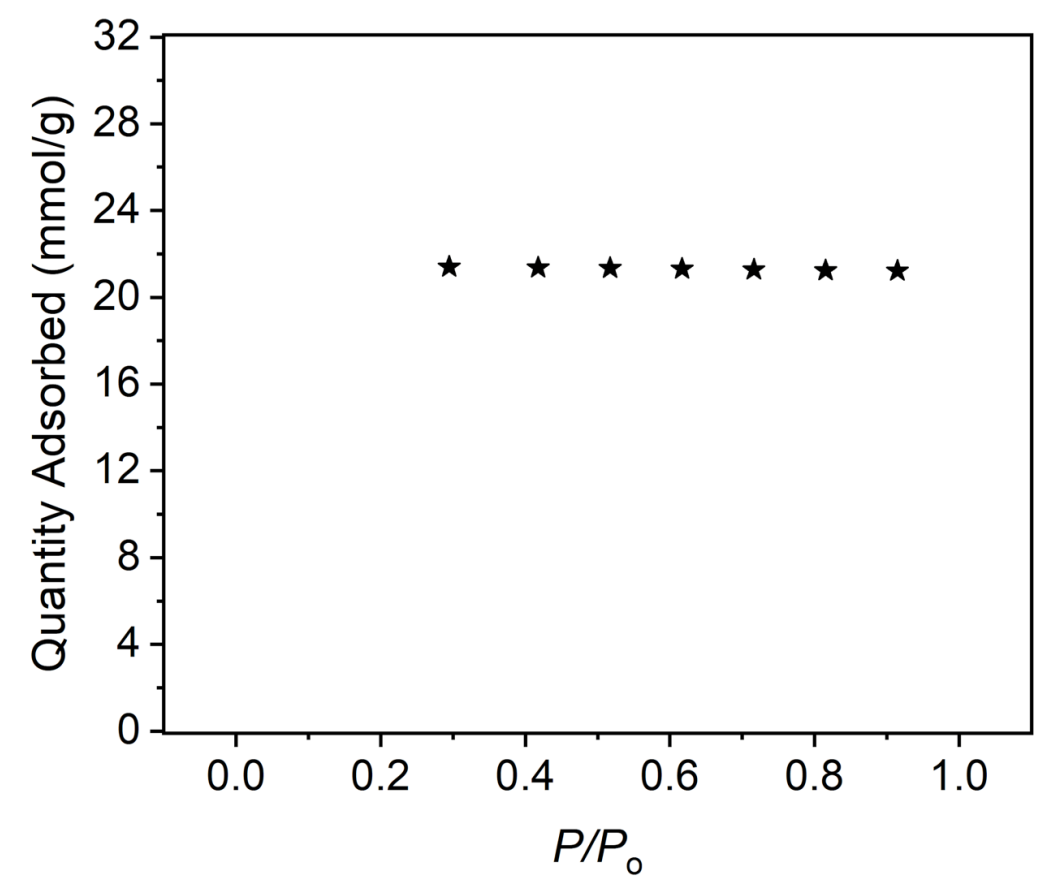

Figure S11. $\mathrm{N}_{2}$ adsorption isotherm at $77 \mathrm{~K}$ of $\mathrm{Co}_{2}(\mathrm{bdc})_{2}$ dabco. 


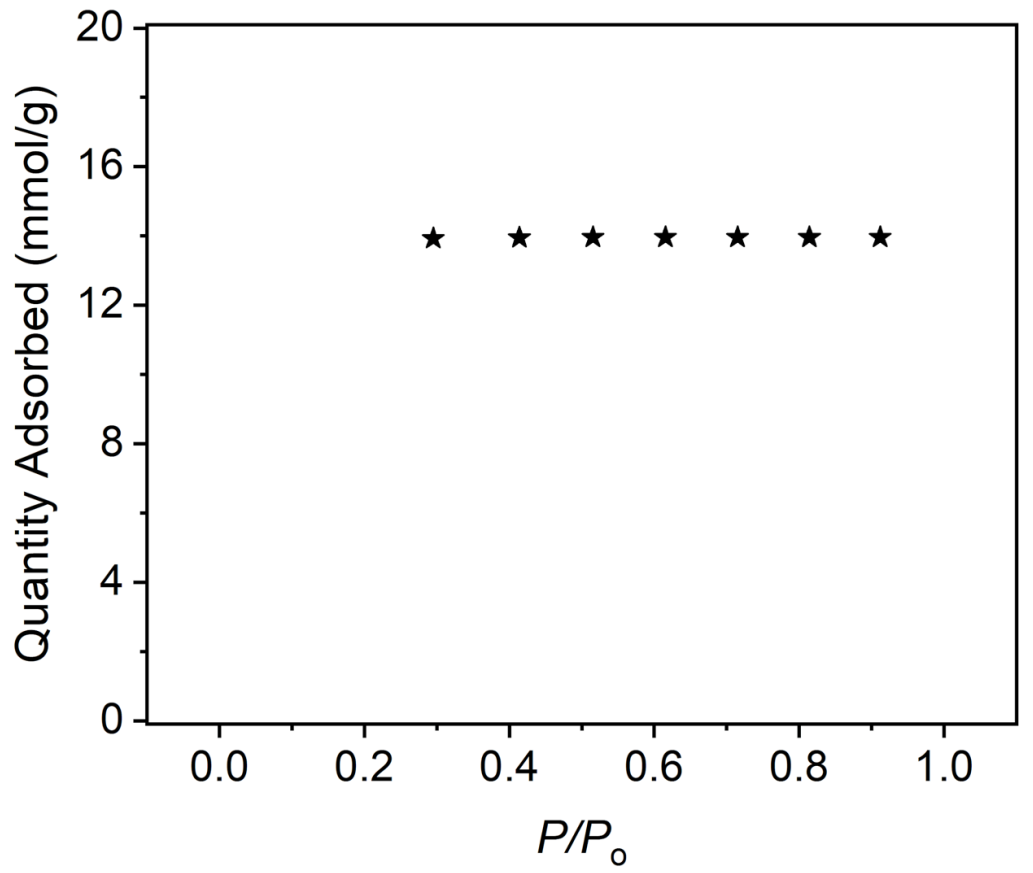

Figure S12. $\mathrm{N}_{2}$ adsorption isotherm at $77 \mathrm{~K}$ of Co-MOF-74. 

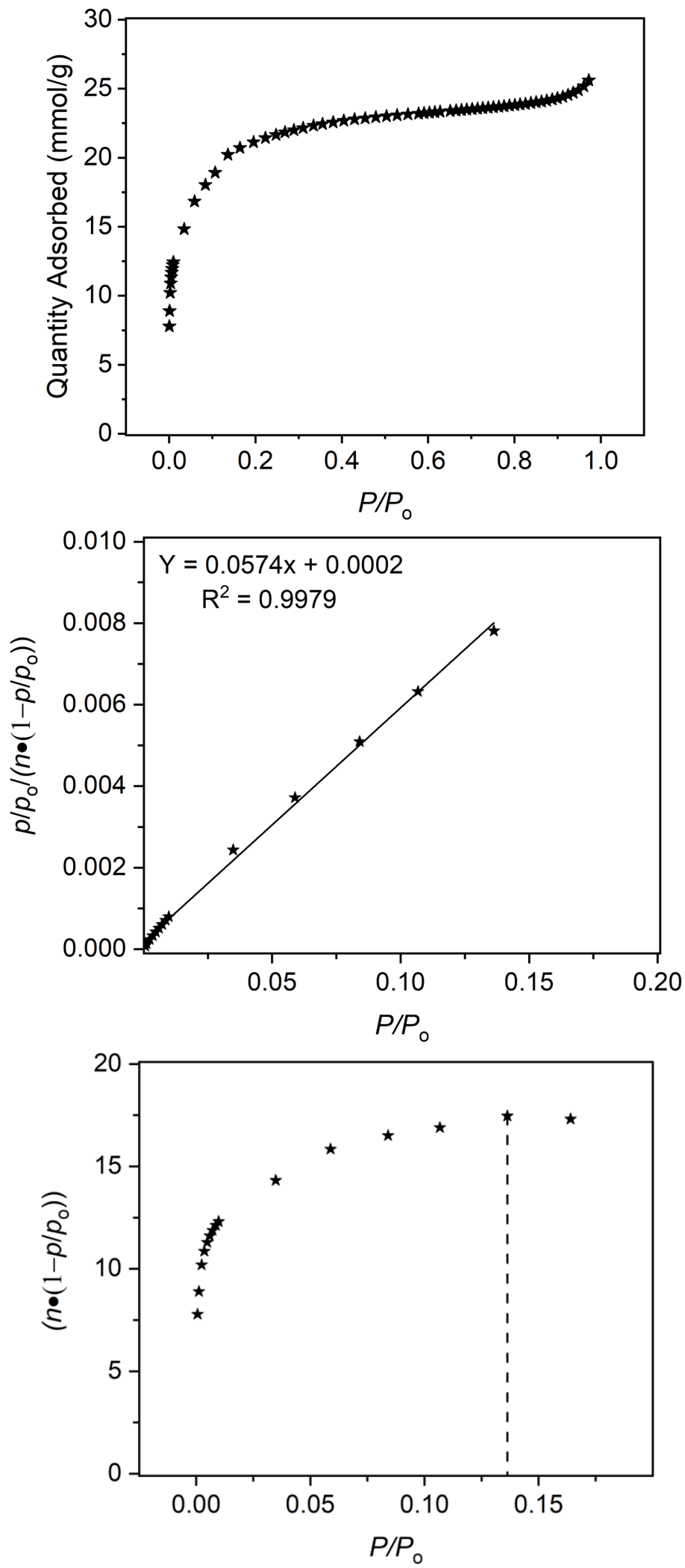

Figure S13. $\mathrm{N}_{2}$ adsorption isotherm at $77 \mathrm{~K}$ of $\mathrm{Cr}-\mathrm{MIL}-100$ with respective BET check plots. 


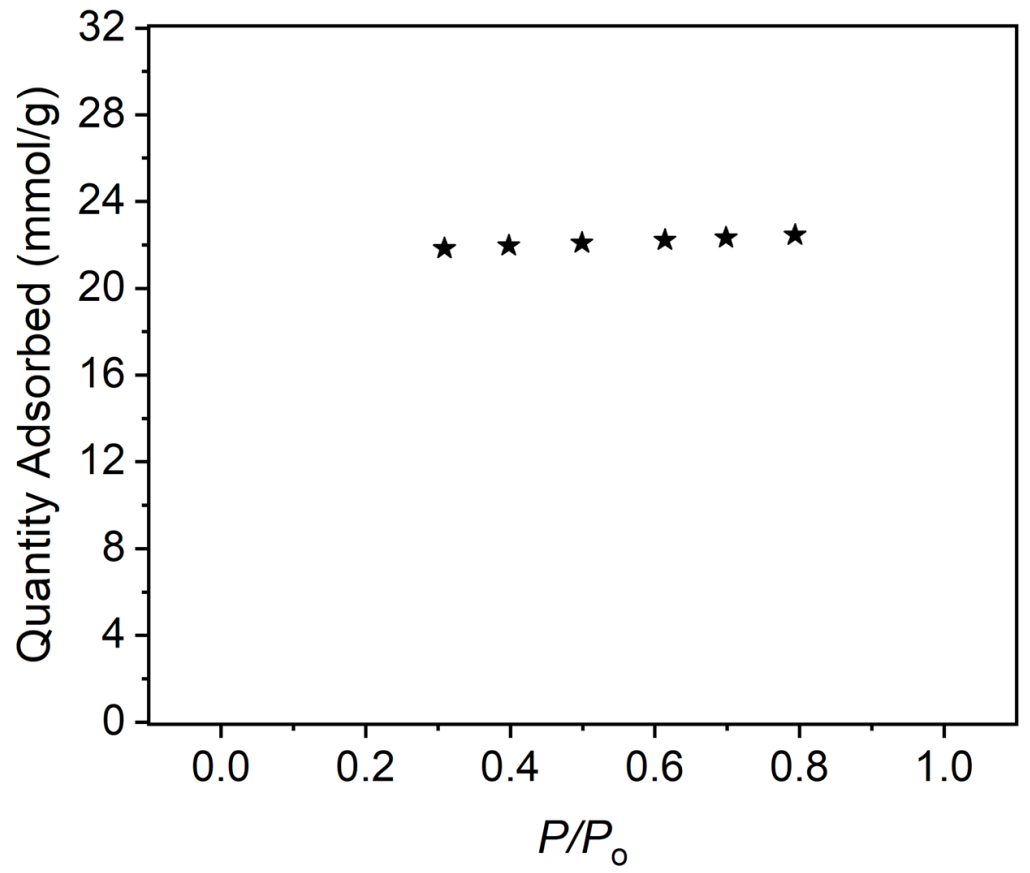

Figure S14. $\mathrm{N}_{2}$ adsorption isotherm at $77 \mathrm{~K}$ of $\mathrm{Cu}_{2}$ (adip).

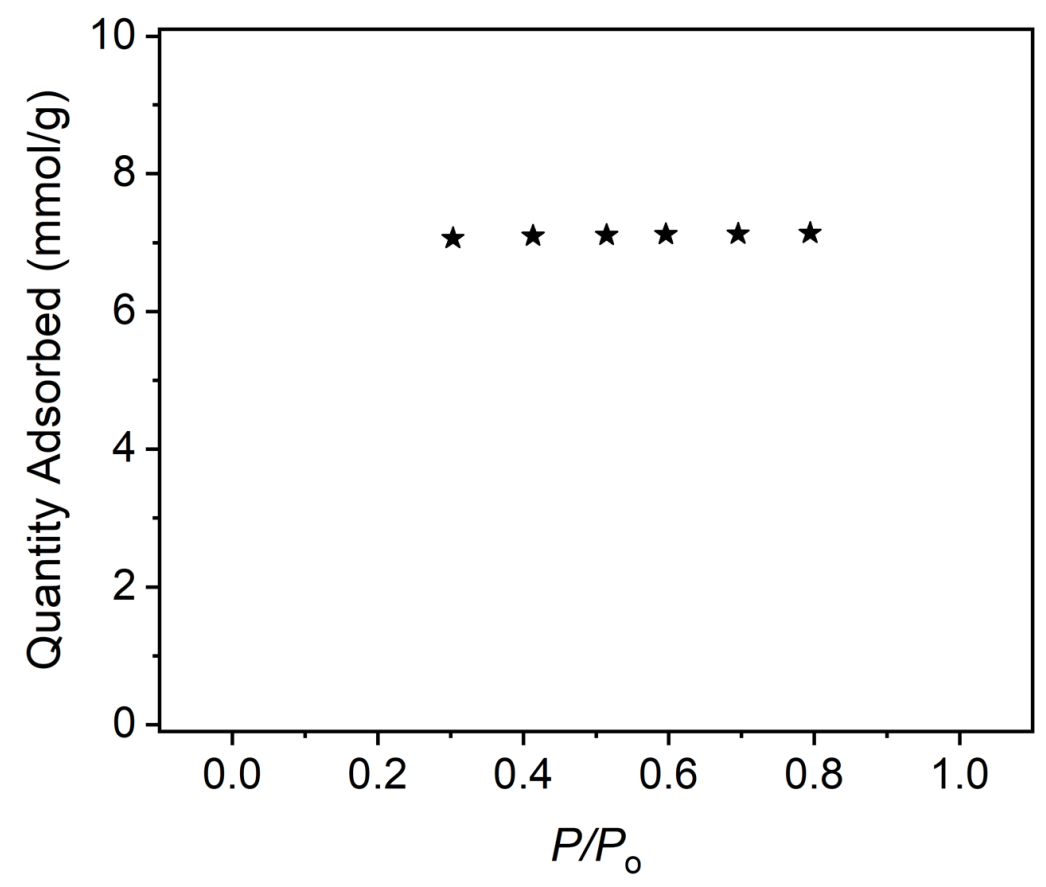

Figure S15. $\mathrm{N}_{2}$ adsorption isotherm at $77 \mathrm{~K}$ of DUT-8 (Zn). 


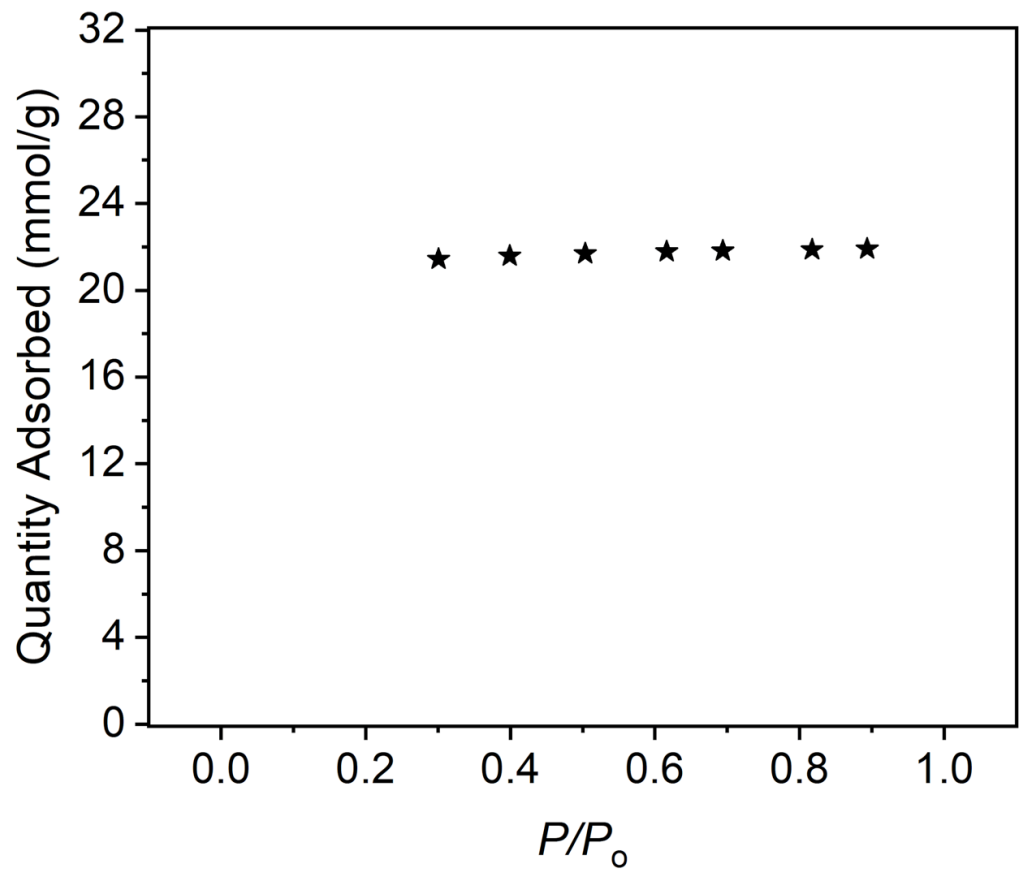

Figure S16. $\mathrm{N}_{2}$ adsorption isotherm at $77 \mathrm{~K}$ of HKUST-1.

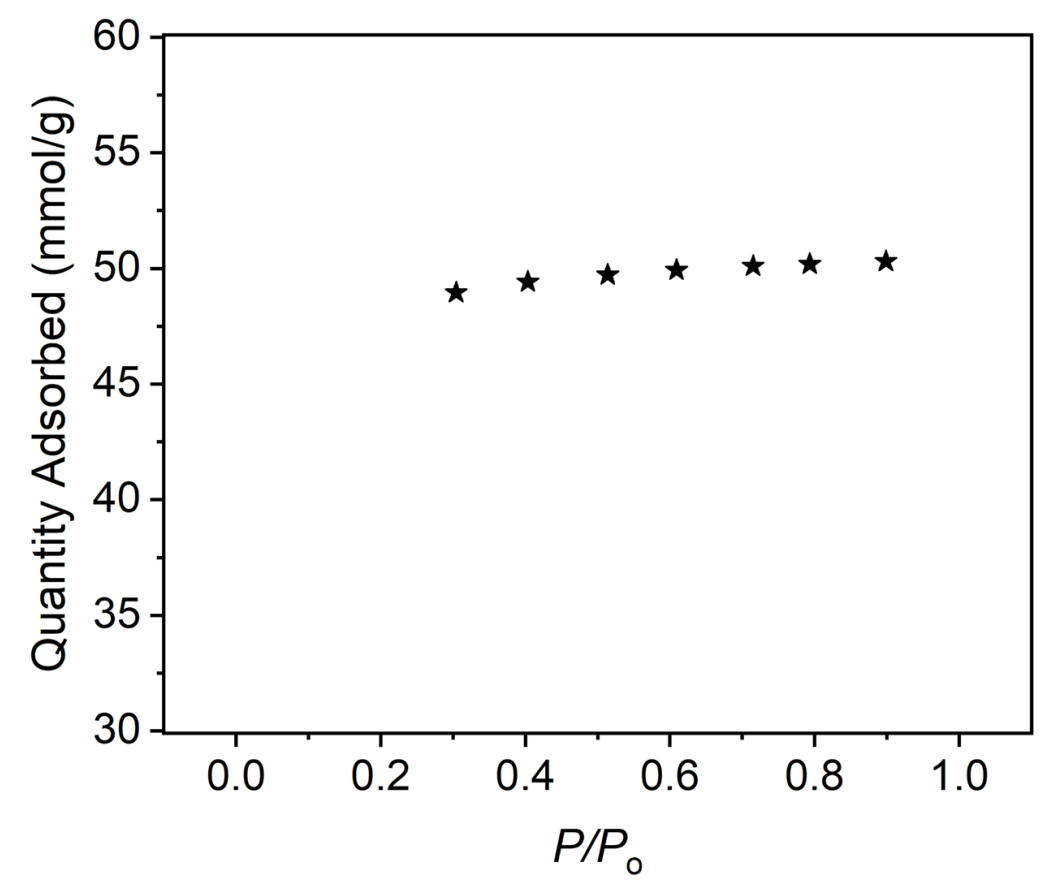

Figure S17. $\mathrm{N}_{2}$ adsorption isotherm at $77 \mathrm{~K}$ of MOF-177. 


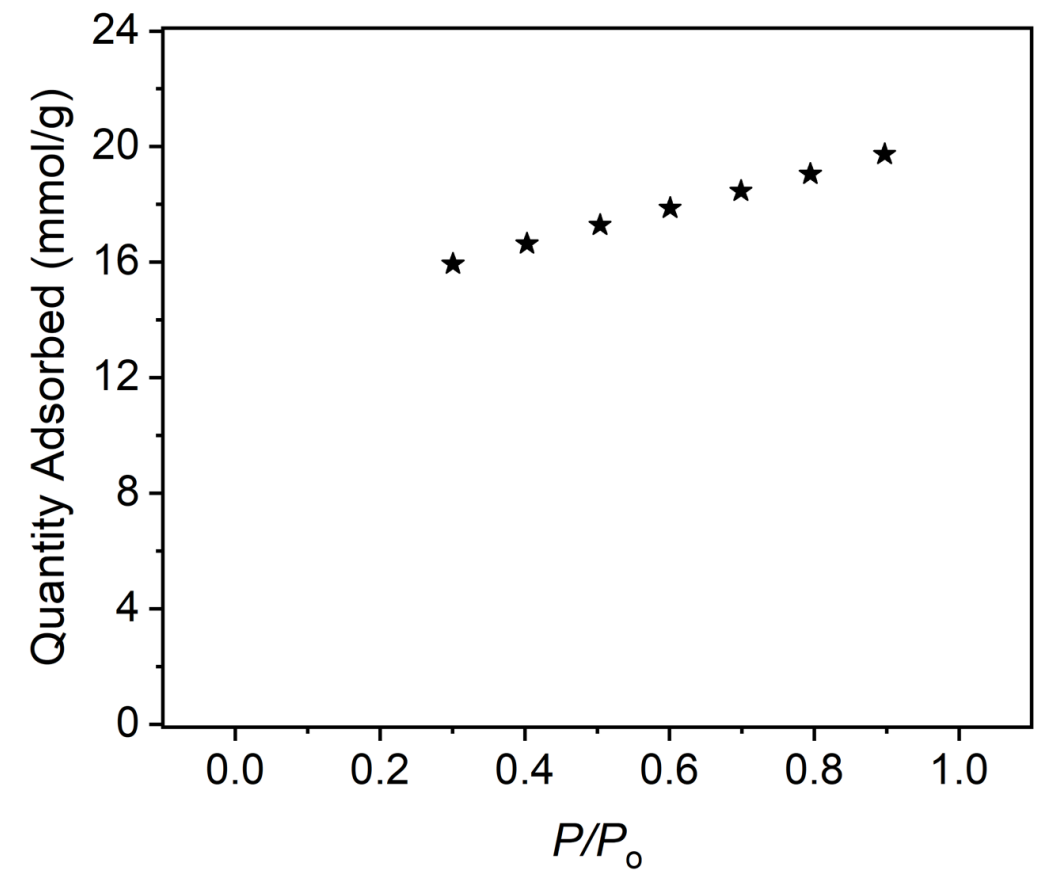

Figure S18. $\mathrm{N}_{2}$ adsorption isotherm at $77 \mathrm{~K}$ of $\mathrm{Mo}_{24}\left({ }^{\mathrm{t}} \mathrm{Bu}-\mathrm{bdc}\right)_{24}$.

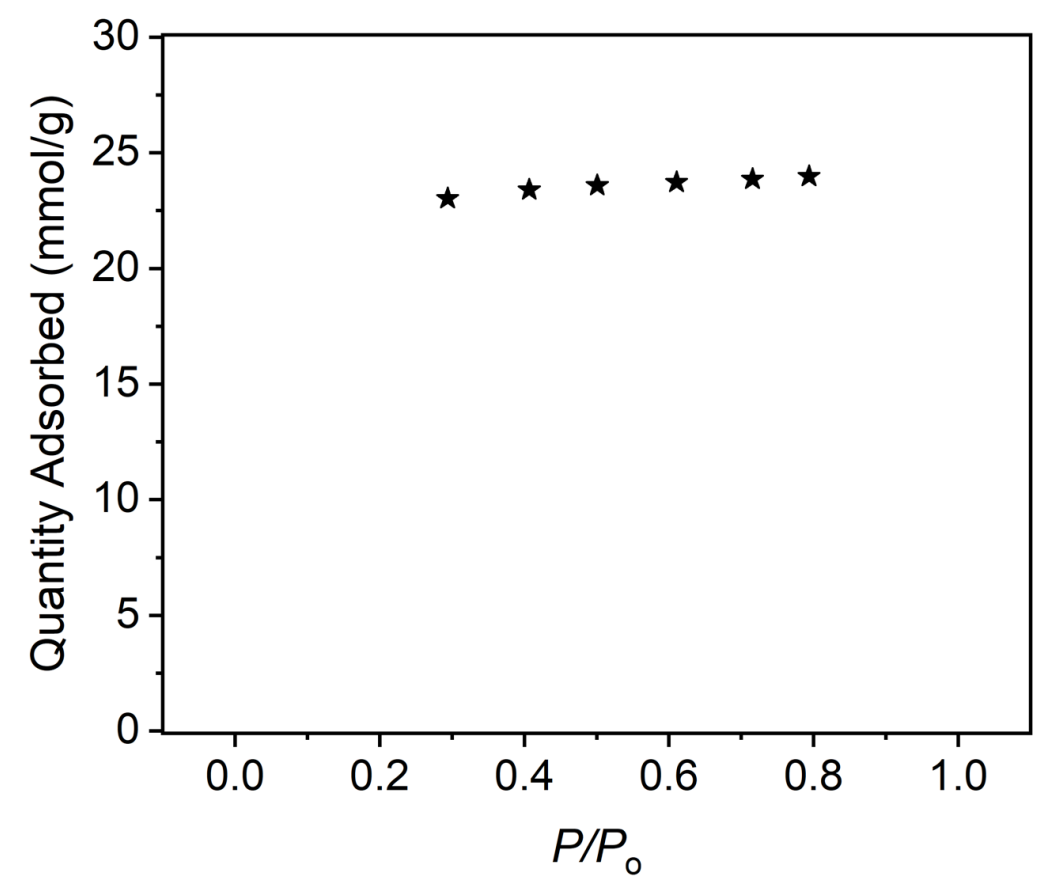

Figure S19. $\mathrm{N}_{2}$ adsorption isotherm at $77 \mathrm{~K}$ of $\mathrm{Ni}(\mathrm{ndc})$ ted $_{0.5}$. 


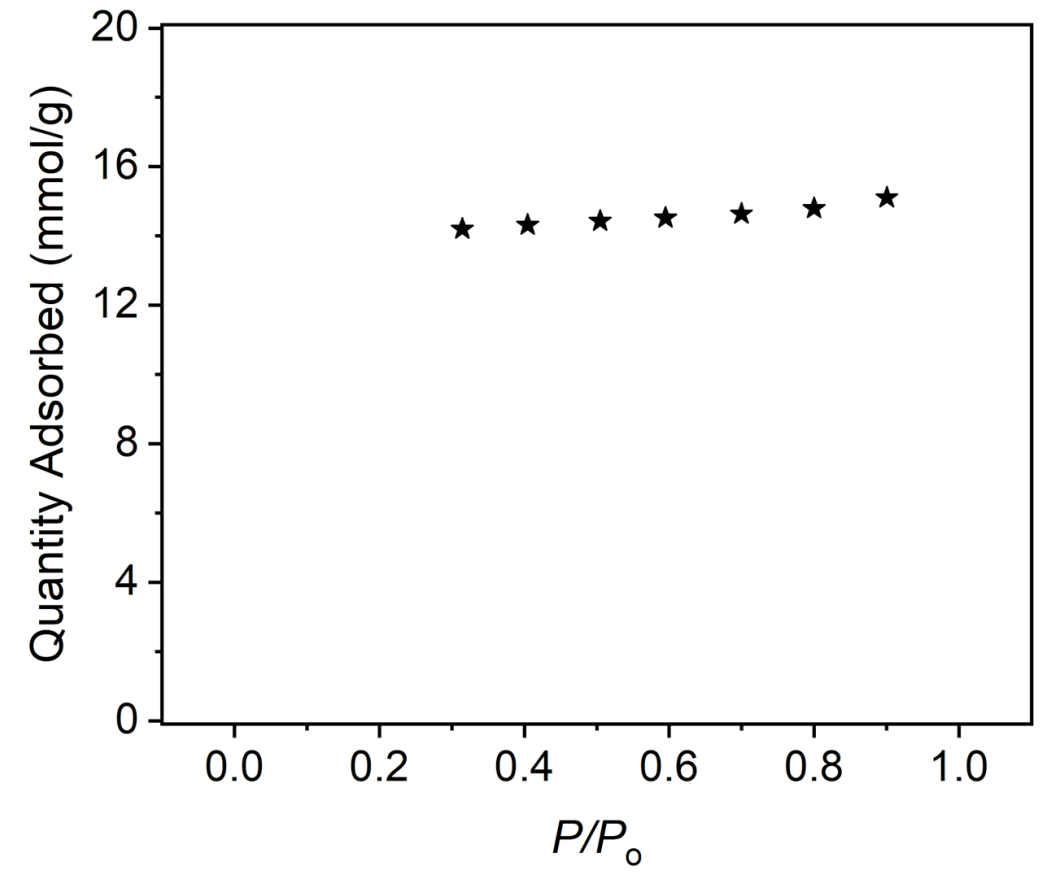

Figure S20. $\mathrm{N}_{2}$ adsorption isotherm at $77 \mathrm{~K}_{\text {of }} \mathrm{Ni}_{2}(m$-dobdc).

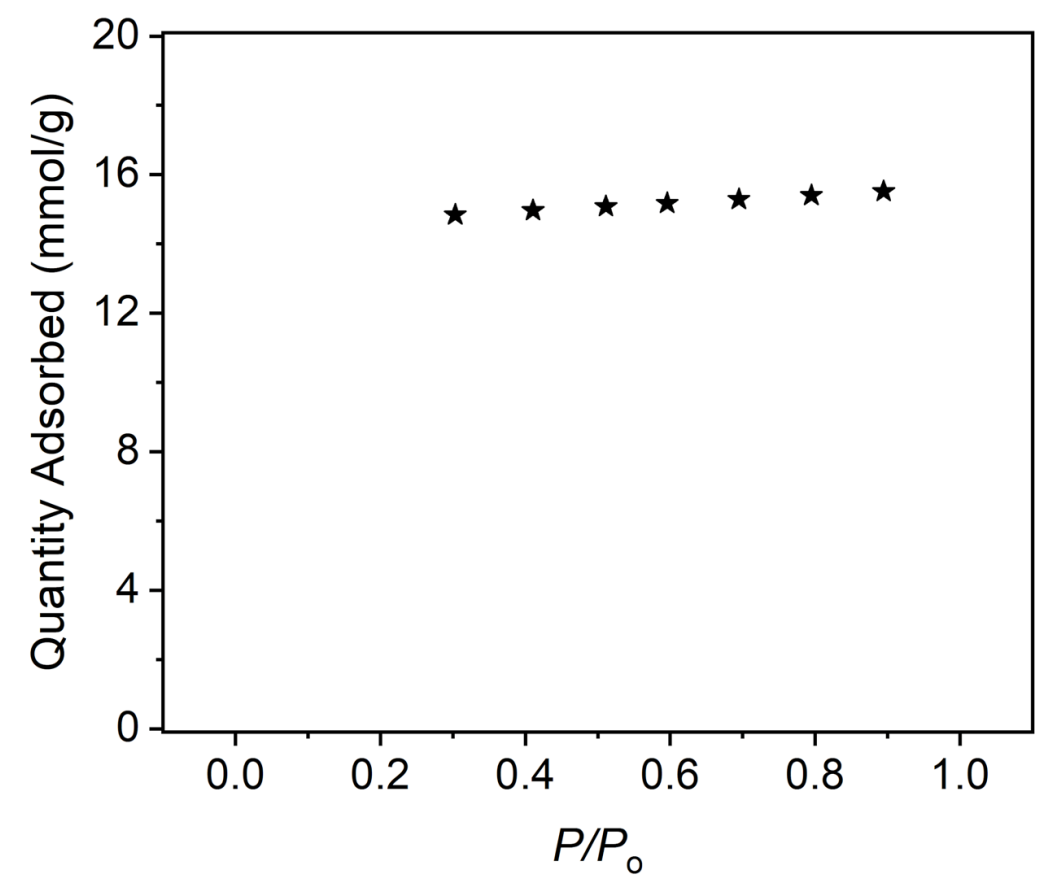

Figure S21. $\mathrm{N}_{2}$ adsorption isotherm at $77 \mathrm{~K}$ of Ni-MOF-74. 


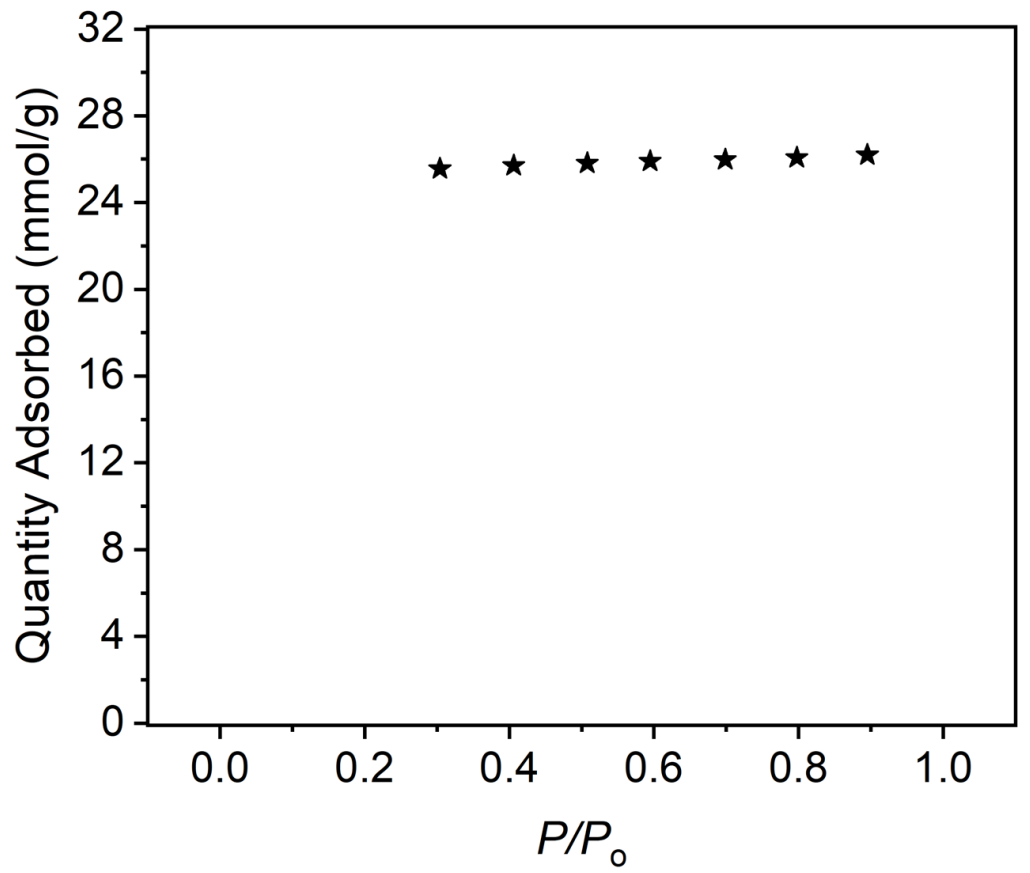

Figure S22. $\mathrm{N}_{2}$ adsorption isotherm at $77 \mathrm{~K}$ of NOTT-101.

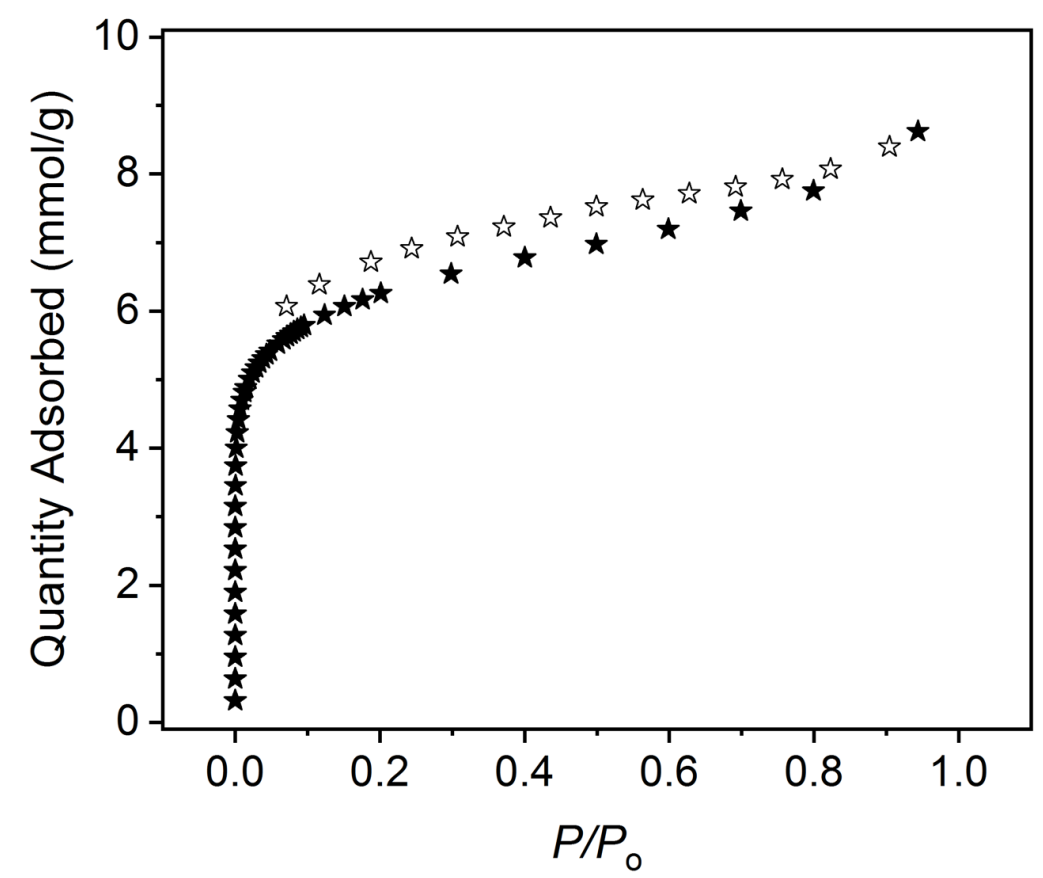

Figure S23. $\mathrm{N}_{2}$ adsorption isotherm at $77 \mathrm{~K}$ of (sc4a)Fe(bdc). 


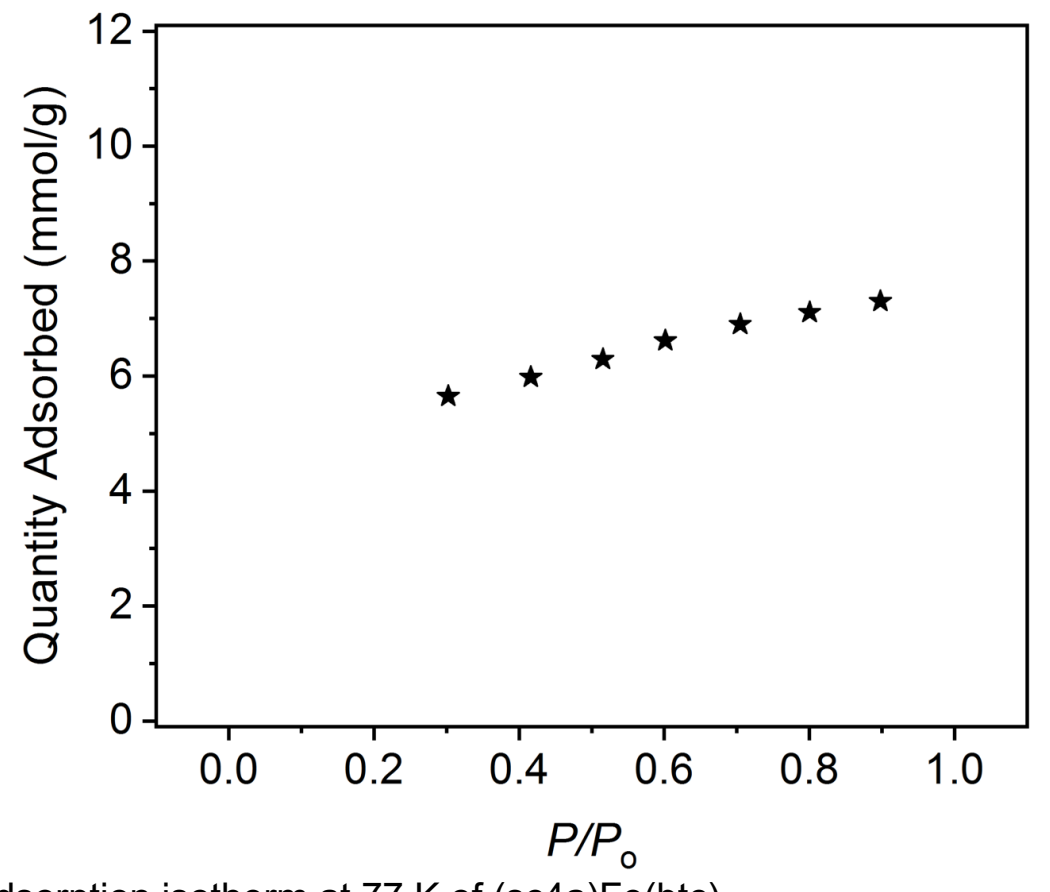

Figure S24. $\mathrm{N}_{2}$ adsorption isotherm at $77 \mathrm{~K}$ of $(\mathrm{sc} 4 \mathrm{a}) \mathrm{Fe}(\mathrm{btc})$.

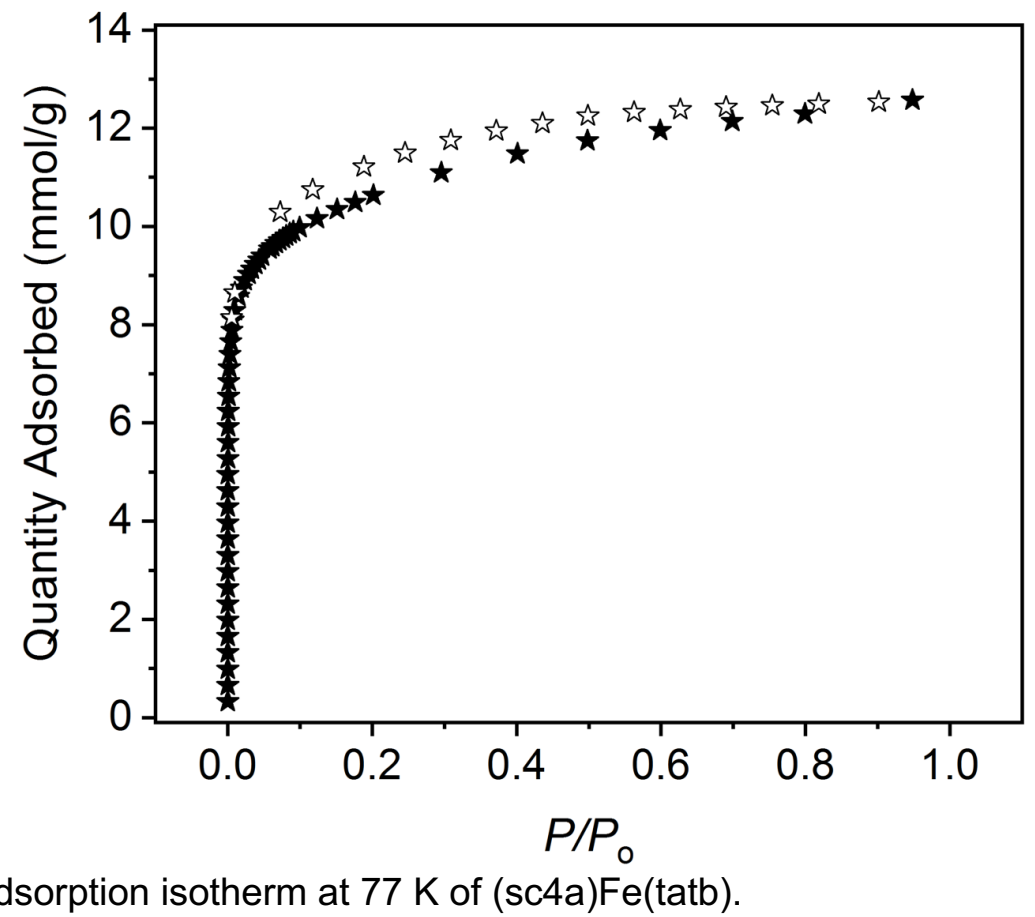

Figure S25. $\mathrm{N}_{2}$ adsorption isotherm at $77 \mathrm{~K}$ of (sc4a)Fe(tatb).

S-23 


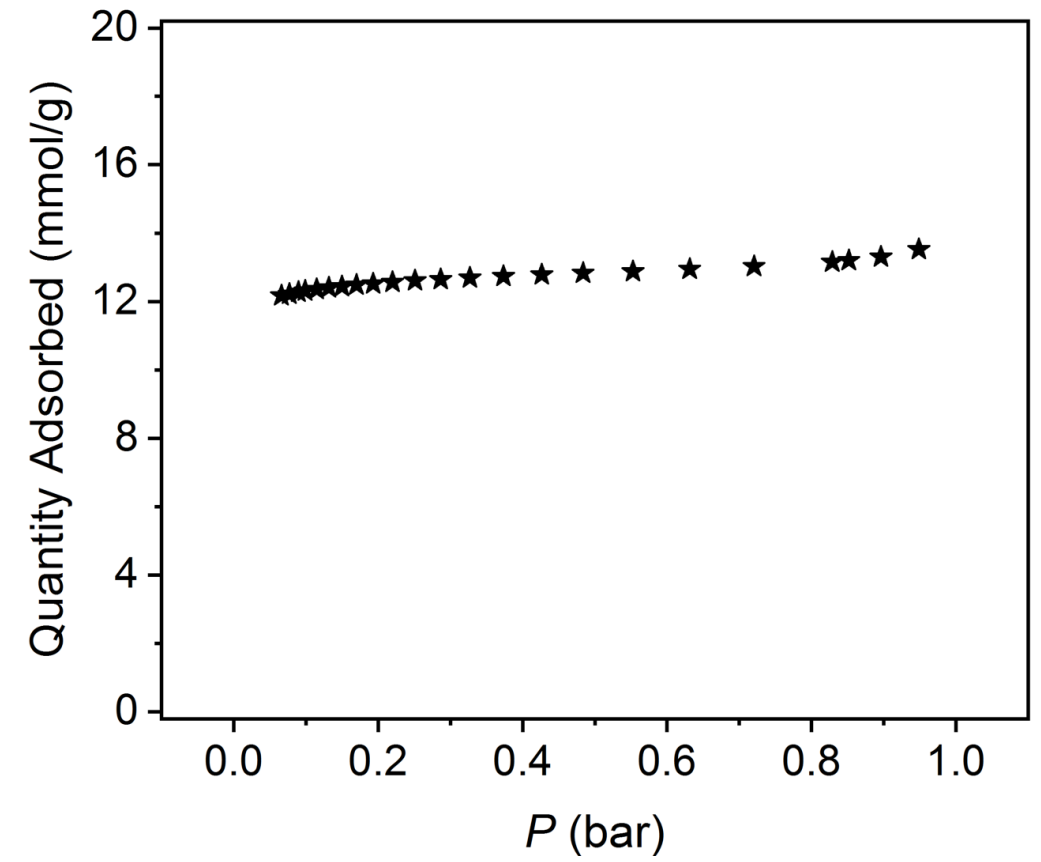

Figure S26. $\mathrm{N}_{2}$ adsorption isotherm at $77 \mathrm{~K}$ of UiO-66 defect free.

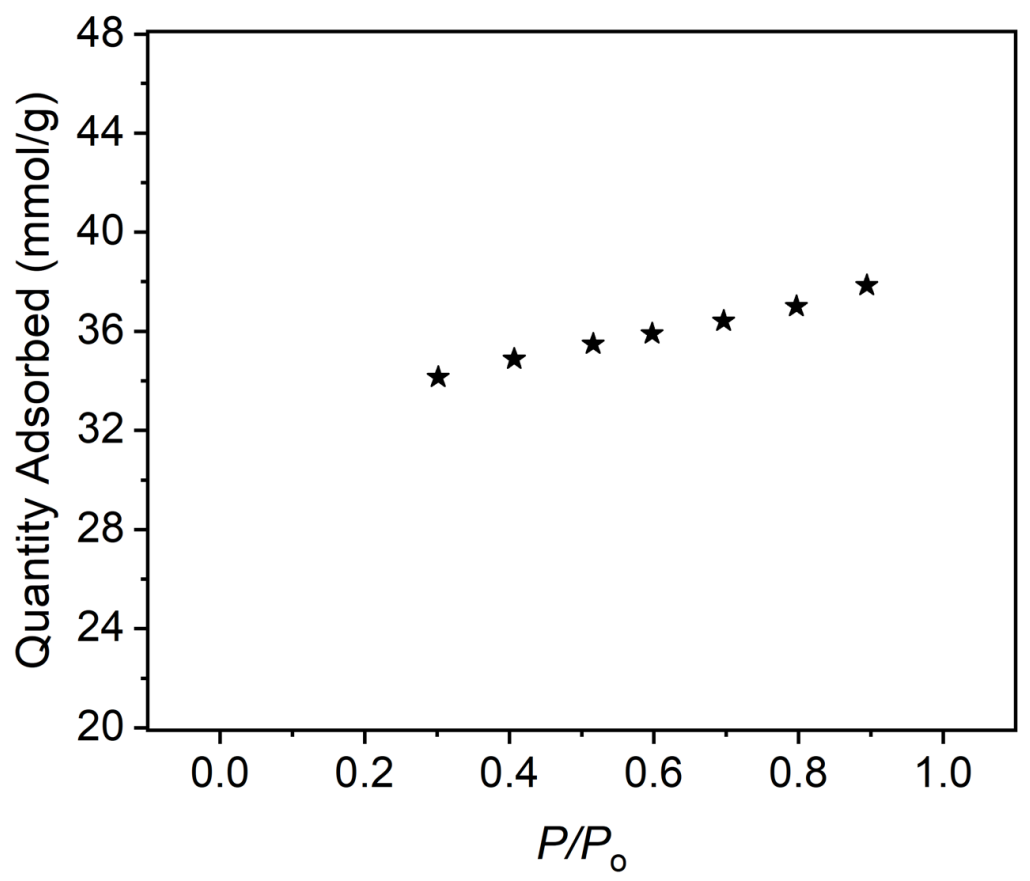

Figure S27. $\mathrm{N}_{2}$ adsorption isotherm at $77 \mathrm{~K}$ of Ti-MIL-101. 


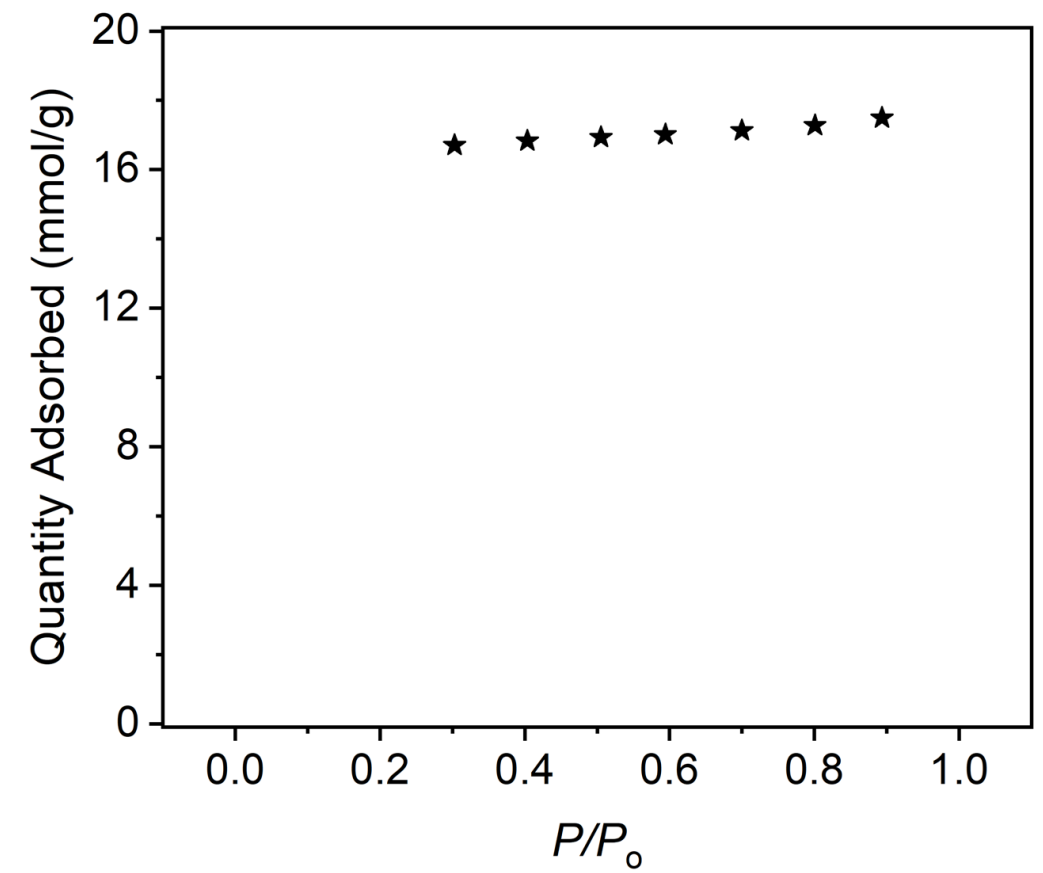

Figure S28. $\mathrm{N}_{2}$ adsorption isotherm at $77 \mathrm{~K}$ of Ti-MIL-125.

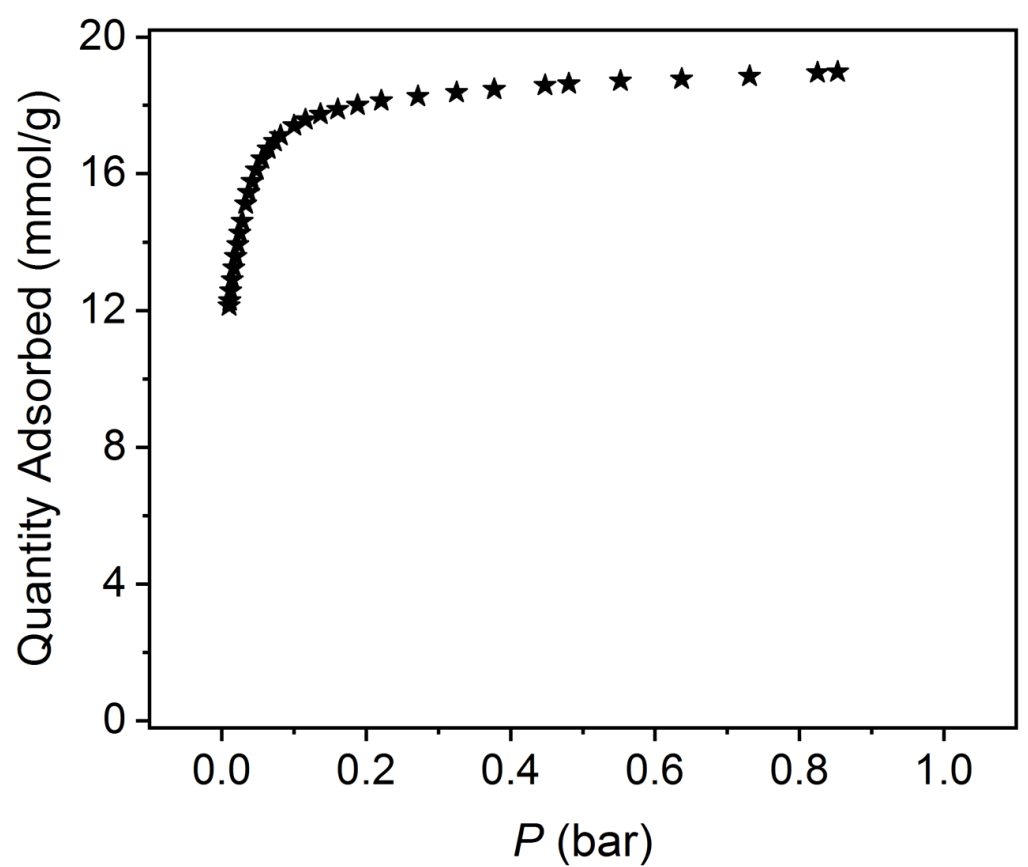

Figure S29. $\mathrm{N}_{2}$ adsorption isotherm at $77 \mathrm{~K}$ of UiO-66 high surface area. 


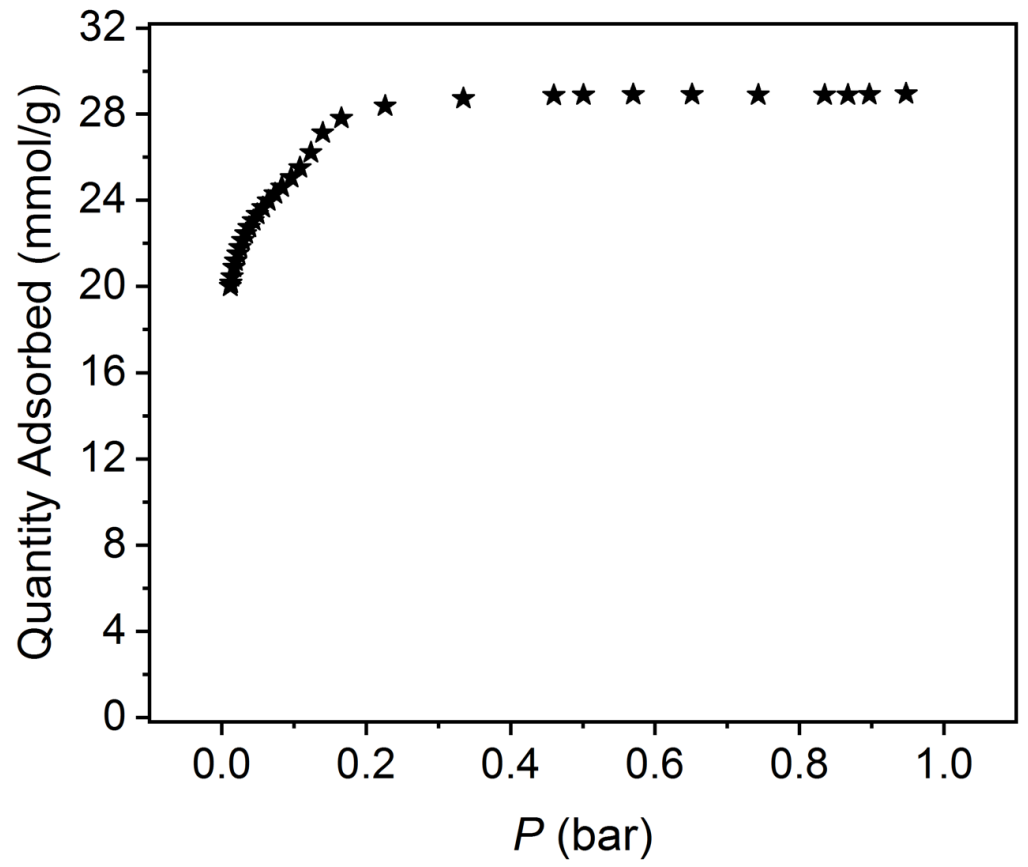

Figure S30. $\mathrm{N}_{2}$ adsorption isotherm at $77 \mathrm{~K}$ of UiO-67.

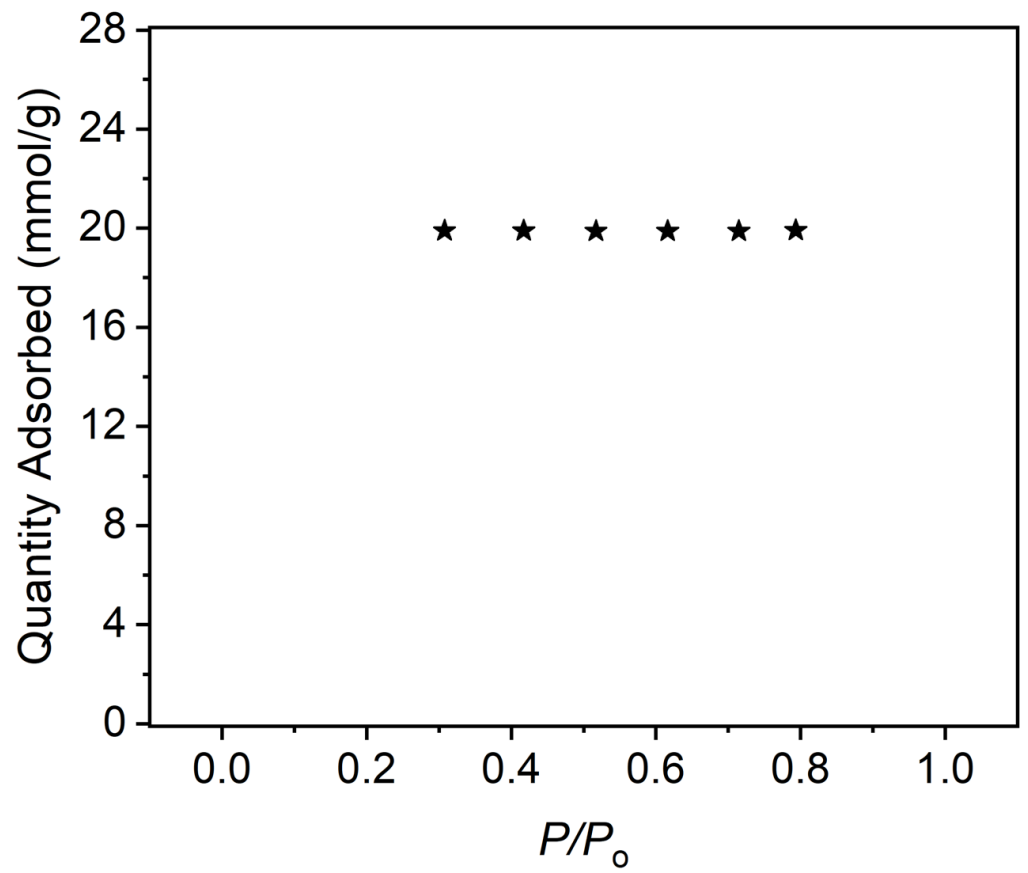

Figure S31. $\mathrm{N}_{2}$ adsorption isotherm at $77 \mathrm{~K}$ of $\mathrm{Zn}\left(\mathrm{bdc}_{\mathrm{C}}\right)_{\text {ted }} \mathrm{d}_{0.5}$. 

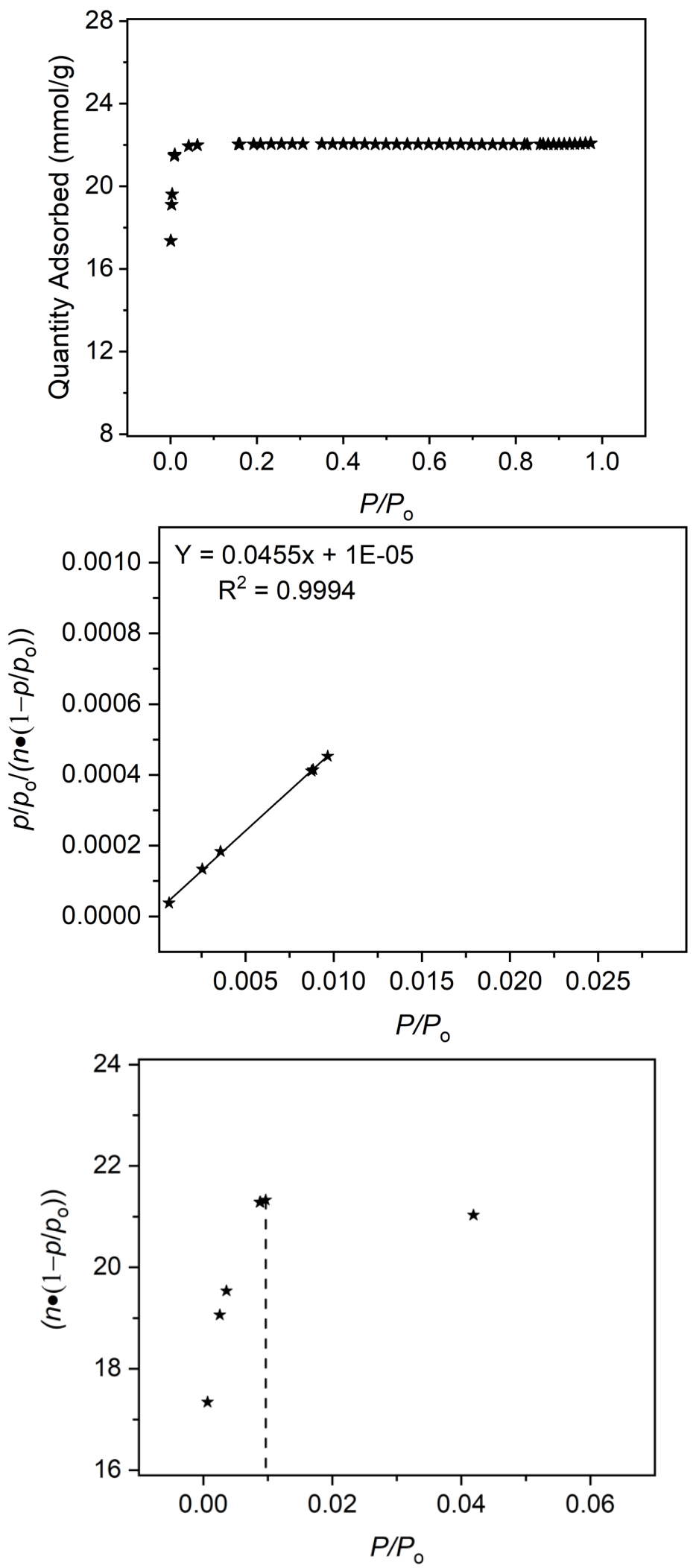

Figure S32. $\mathrm{N}_{2}$ adsorption isotherm at $77 \mathrm{~K}$ of $\mathrm{Zn}_{2}(\mathrm{bdc})_{2}$ dabco with respective BET check plots. 


\section{Methane Adsorption Isotherms}

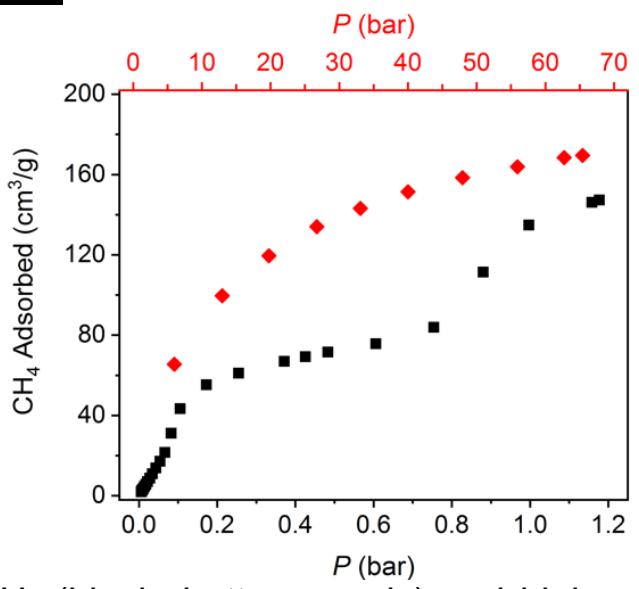

Figure S33. Low-pressure $\mathrm{CH}_{4}$ (black, bottom x-axis) and high-pressure $\mathrm{CH}_{4}$ (red, top x-axis) adsorption isotherms at $195 \mathrm{~K}$ and $298 \mathrm{~K}$ respectively, for Al-MIL-53.

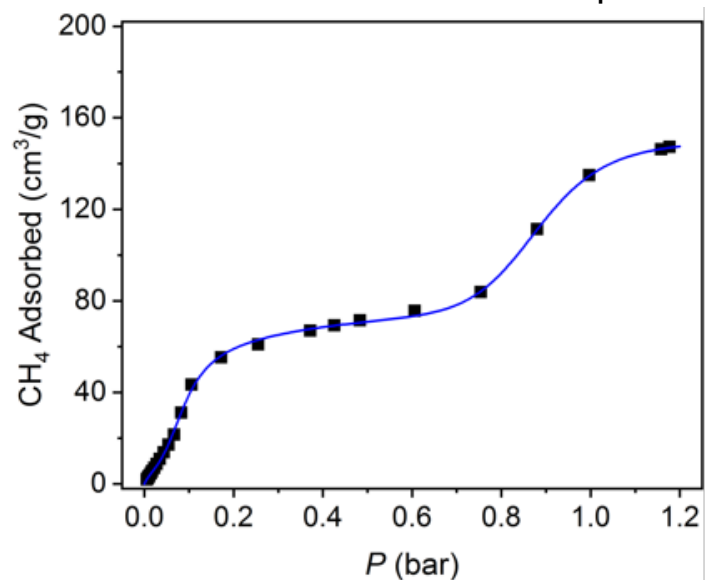

\begin{tabular}{|c|c|}
\hline $\mathrm{q}_{\mathrm{sat}, 1}$ & 36.994 \\
$\mathrm{~b}_{1}$ & 3202.087 \\
\hline $\mathrm{v}_{1}$ & 3.349 \\
\hline $\mathrm{q}_{\mathrm{sat}, 2}$ & 71.364 \\
\hline $\mathrm{b}_{2}$ & 4.456 \\
\hline $\mathrm{v}_{2}$ & 11.853 \\
\hline $\mathrm{q}_{\mathrm{sat}, 3}$ & 50 \\
\hline $\mathrm{b}_{3}$ & 3.863 \\
\hline $\mathrm{v}_{3}$ & 0.87 \\
\hline
\end{tabular}

Figure S34. Low pressure $\mathrm{CH}_{4}$ isotherm with fit (left) and corresponding fitting parameters (right) for Al-MIL-53 at $195 \mathrm{~K}$.

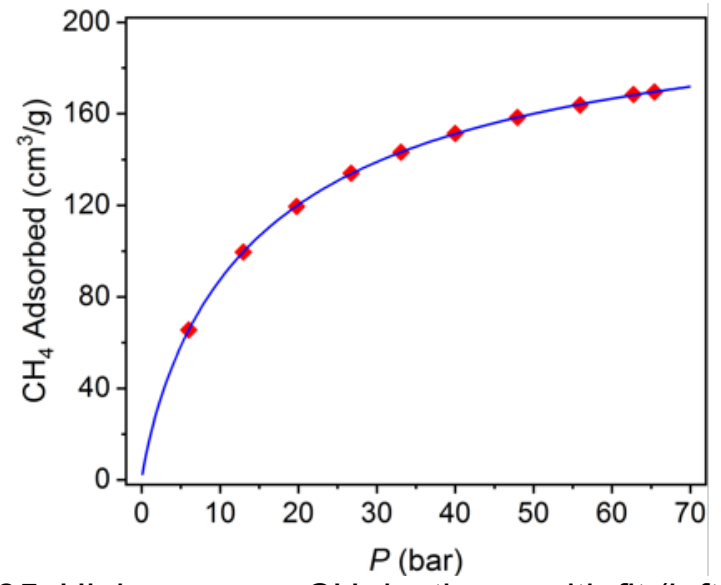

\begin{tabular}{|c|c|}
\hline $\mathrm{q}_{\mathrm{sat}, 1}$ & 219.450 \\
$\mathrm{~b}_{1}$ & 0.089 \\
\hline $\mathrm{v}_{1}$ & 0.870 \\
\hline $\mathrm{q}_{\mathrm{sat}, 2}$ & 0 \\
\hline $\mathrm{b}_{2}$ & 0 \\
\hline $\mathrm{v}_{2}$ & 0 \\
\hline $\mathrm{q}_{\mathrm{sat}, 3}$ & 0 \\
\hline $\mathrm{b}_{3}$ & 0 \\
\hline $\mathrm{v}_{3}$ & 0 \\
\hline
\end{tabular}

Figure S35. High pressure $\mathrm{CH}_{4}$ isotherm with fit (left) and corresponding fitting parameters (right) for Al-MIL-53 at $298 \mathrm{~K}$. 


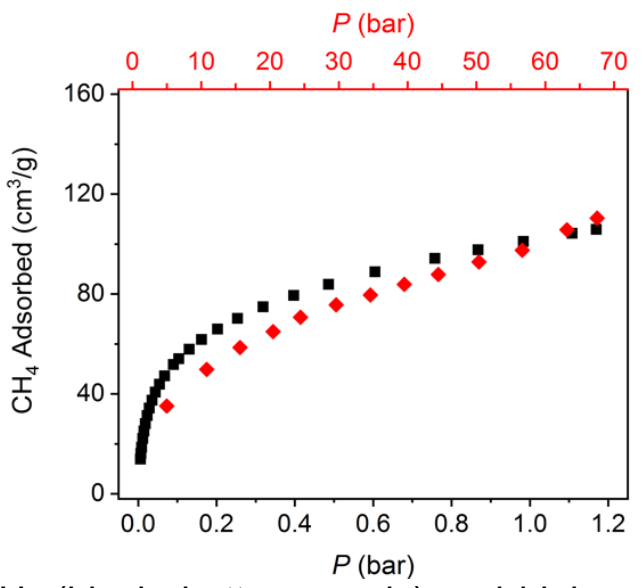

Figure S36. Low-pressure $\mathrm{CH}_{4}$ (black, bottom x-axis) and high-pressure $\mathrm{CH}_{4}$ (red, top x-axis) adsorption isotherms at $195 \mathrm{~K}$ and $298 \mathrm{~K}$ respectively, for CC3-R.

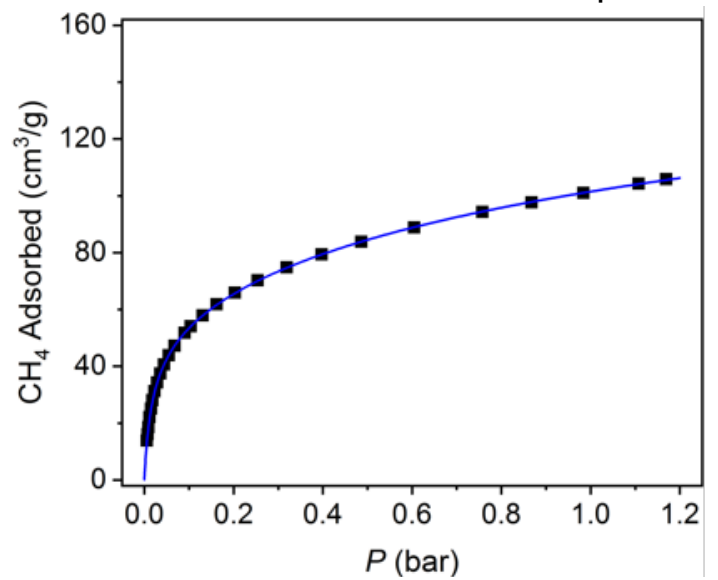

\begin{tabular}{|c|c|}
\hline $\mathrm{q}_{\mathrm{sat}, 1}$ & 30.200 \\
$\mathrm{~b}_{1}$ & 104.087 \\
\hline $\mathrm{v}_{1}$ & 1.108 \\
\hline $\mathrm{q}_{\mathrm{sat}, 2}$ & 190.200 \\
\hline $\mathrm{b}_{2}$ & 0.603 \\
\hline $\mathrm{v}_{2}$ & 0.571 \\
\hline $\mathrm{q}_{\mathrm{sat}, 3}$ & 0 \\
\hline $\mathrm{b}_{3}$ & 0 \\
\hline $\mathrm{v}_{3}$ & 0 \\
\hline
\end{tabular}

Figure S37. Low pressure $\mathrm{CH}_{4}$ isotherm with fit (left) and corresponding fitting parameters (right) for CC3-R at $195 \mathrm{~K}$.

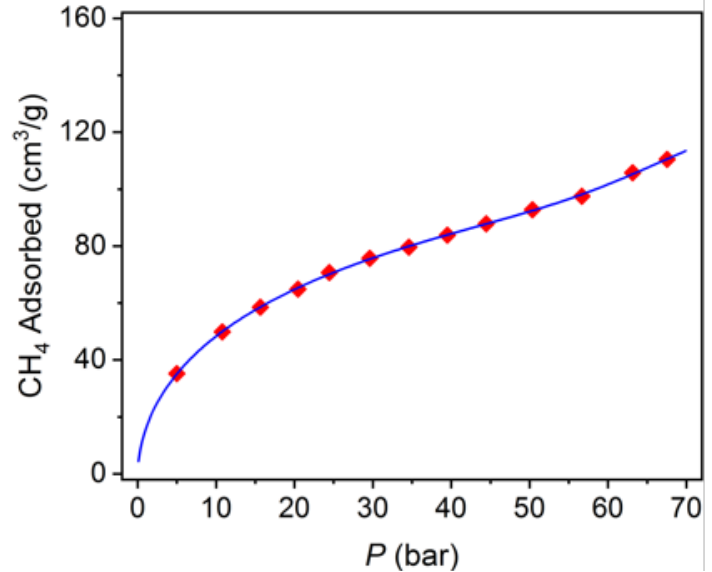

\begin{tabular}{|c|c|}
\hline $\mathrm{q}_{\mathrm{sat}, 1}$ & 23.684 \\
$\mathrm{~b}_{1}$ & $1.13 \mathrm{e}-16$ \\
\hline $\mathrm{v}_{1}$ & 8.658 \\
\hline $\mathrm{q}_{\mathrm{sat}, 2}$ & 221.753 \\
\hline $\mathrm{b}_{2}$ & 0.075 \\
\hline $\mathrm{v}_{2}$ & 0.567 \\
\hline $\mathrm{q}_{\mathrm{sat}, 3}$ & 0 \\
\hline $\mathrm{b}_{3}$ & 0 \\
\hline $\mathrm{v}_{3}$ & 0 \\
\hline
\end{tabular}

Figure S38. High pressure $\mathrm{CH}_{4}$ isotherm with fit (left) and corresponding fitting parameters (right) for CC3-R at $298 \mathrm{~K}$. 


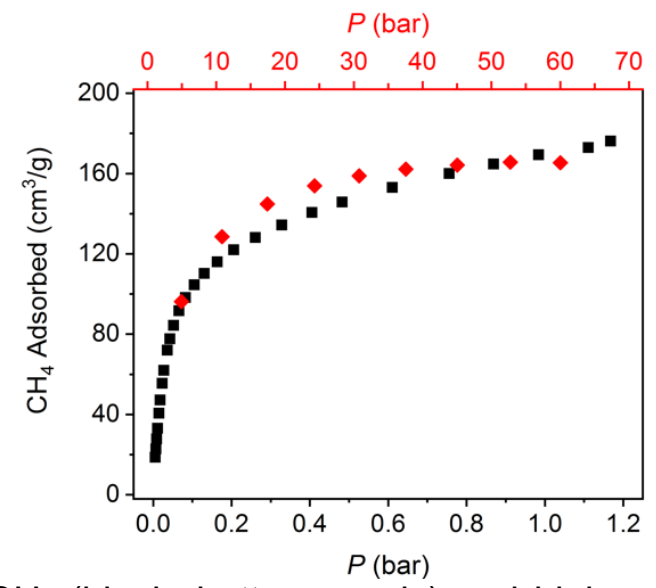

Figure S39. Low-pressure $\mathrm{CH}_{4}$ (black, bottom x-axis) and high-pressure $\mathrm{CH}_{4}$ (red, top x-axis) adsorption isotherms at $195 \mathrm{~K}$ and $298 \mathrm{~K}$ respectively, for $\mathrm{Co}_{2}(m$-dobdc).

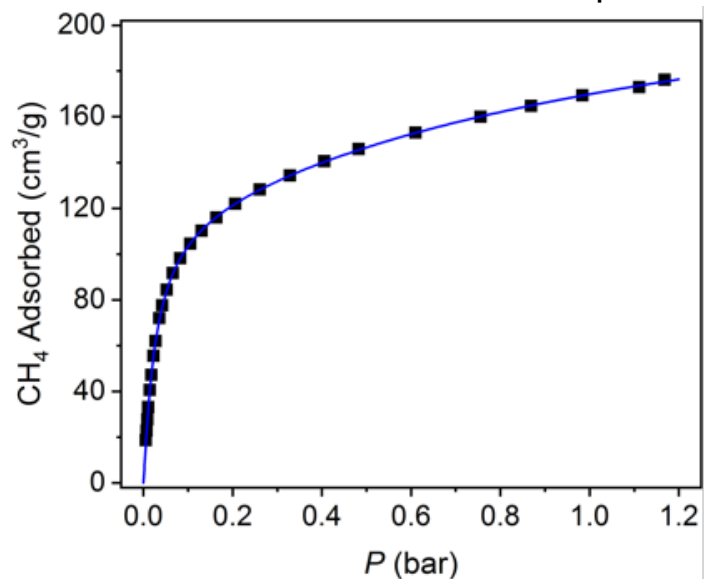

\begin{tabular}{|c|c|}
\hline $\mathrm{q}_{\mathrm{sat}, 1}$ & 114.408 \\
$\mathrm{~b}_{1}$ & 68.319 \\
\hline $\mathrm{v}_{1}$ & 1.153 \\
\hline $\mathrm{q}_{\mathrm{sat}, 2}$ & 131.622 \\
\hline $\mathrm{b}_{2}$ & 0.766 \\
\hline $\mathrm{v}_{2}$ & 1.036 \\
\hline $\mathrm{q}_{\mathrm{sat}, 3}$ & 0 \\
\hline $\mathrm{b}_{3}$ & 0 \\
\hline $\mathrm{v}_{3}$ & 0 \\
\hline
\end{tabular}

Figure S40. Low pressure $\mathrm{CH}_{4}$ isotherm with fit (left) and corresponding fitting parameters (right) for $\mathrm{Co}_{2}(m$-dobdc) at $195 \mathrm{~K}$.

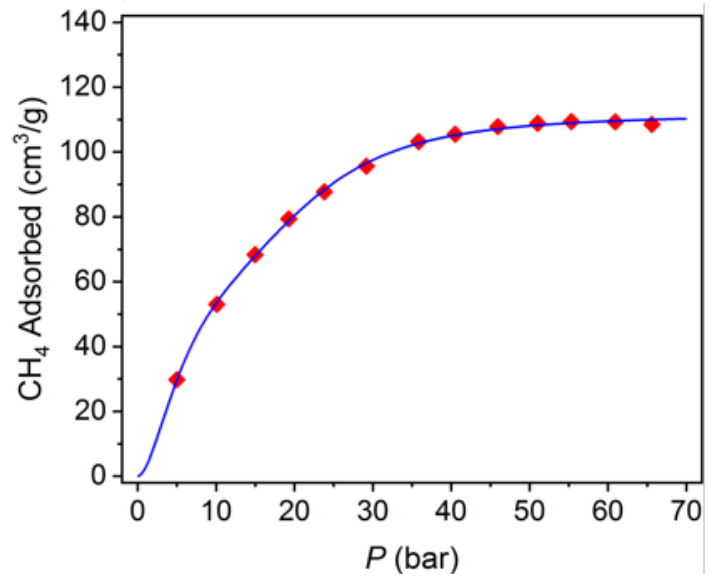

\begin{tabular}{|c|c|}
\hline $\mathrm{q}_{\mathrm{sat}, 1}$ & 28.796 \\
$\mathrm{~b}_{1}$ & $4.201 \mathrm{e}-4$ \\
\hline $\mathrm{v}_{1}$ & 2.560 \\
\hline $\mathrm{q}_{\mathrm{sat}, 2}$ & 76.656 \\
\hline $\mathrm{b}_{2}$ & 0.119 \\
\hline $\mathrm{v}_{2}$ & 1.53564 \\
\hline $\mathrm{q}_{\mathrm{sat}, 3}$ & 0 \\
\hline $\mathrm{b}_{3}$ & 0 \\
\hline $\mathrm{v}_{3}$ & 0 \\
\hline
\end{tabular}

Figure S41. High pressure $\mathrm{CH}_{4}$ isotherm with fit (left) and corresponding fitting parameters (right) for $\mathrm{Co}_{2}(m-$ dobdc $)$ at $298 \mathrm{~K}$. 


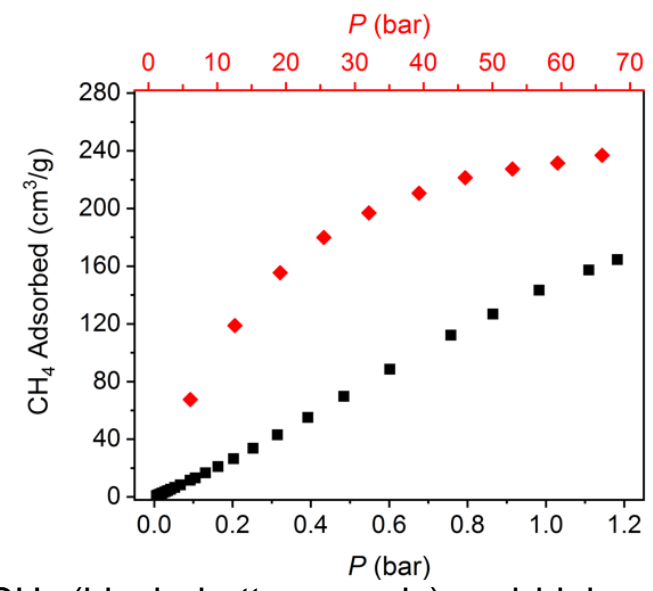

Figure S42. Low-pressure $\mathrm{CH}_{4}$ (black, bottom x-axis) and high-pressure $\mathrm{CH}_{4}$ (red, top x-axis) adsorption isotherms at $195 \mathrm{~K}$ and $298 \mathrm{~K}$ respectively, for $\mathrm{Co}_{2}(\mathrm{bdc})_{2} \mathrm{dabco}$.

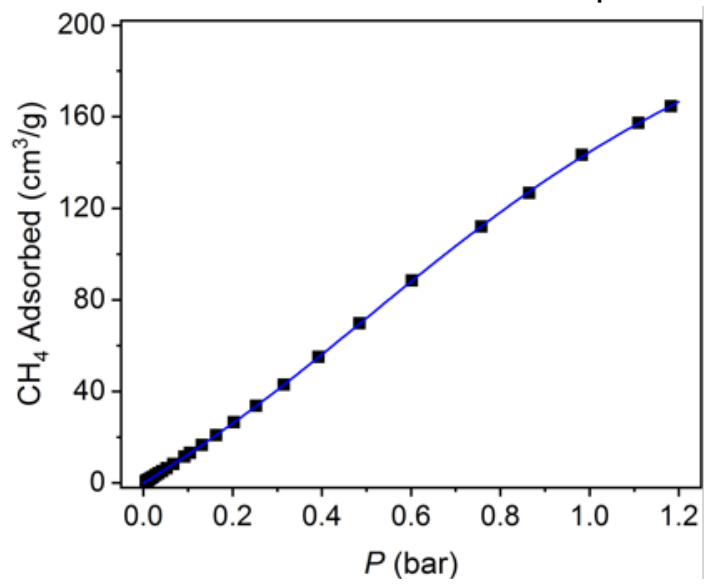

\begin{tabular}{|c|c|}
\hline $\mathrm{q}_{\mathrm{sat}, 1}$ & 171.384 \\
$\mathrm{~b}_{1}$ & 0.914 \\
\hline $\mathrm{v}_{1}$ & 2.105 \\
\hline $\mathrm{q}_{\mathrm{sat}, 2}$ & 119.434 \\
\hline $\mathrm{b}_{2}$ & 1.109 \\
\hline $\mathrm{v}_{2}$ & 1.028 \\
\hline $\mathrm{q}_{\mathrm{sat}, 3}$ & 0 \\
\hline $\mathrm{b}_{3}$ & 0 \\
\hline $\mathrm{v}_{3}$ & 0 \\
\hline
\end{tabular}

Figure S43. Low pressure $\mathrm{CH}_{4}$ isotherm with fit (left) and corresponding fitting parameters (right) for $\mathrm{Co}_{2}(\mathrm{bdc})_{2} \mathrm{dabco}$ at $195 \mathrm{~K}$.

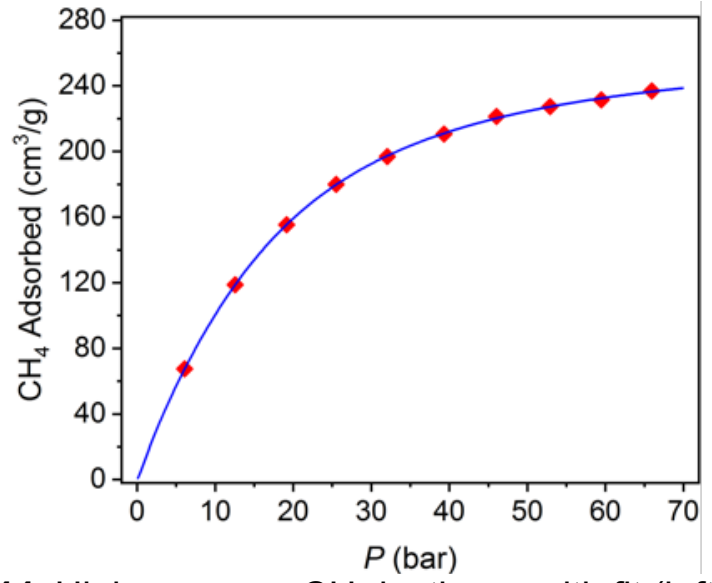

\begin{tabular}{|c|c|}
\hline $\mathrm{q}_{\mathrm{sat}, 1}$ & 55.207 \\
$\mathrm{~b}_{1}$ & 0.214 \\
\hline $\mathrm{v}_{1}$ & 1.183 \\
\hline $\mathrm{q}_{\mathrm{sat}, 2}$ & 209.032 \\
\hline $\mathrm{b}_{2}$ & 0.011 \\
\hline $\mathrm{v}_{2}$ & 1.541 \\
\hline $\mathrm{q}_{\mathrm{sat}, 3}$ & 0 \\
\hline $\mathrm{b}_{3}$ & 0 \\
\hline $\mathrm{v}_{3}$ & 0 \\
\hline
\end{tabular}

Figure S44. High pressure $\mathrm{CH}_{4}$ isotherm with fit (left) and corresponding fitting parameters (right) for $\mathrm{Co}_{2}(\mathrm{bdc})_{2} \mathrm{dabco}$ at $298 \mathrm{~K}$. 


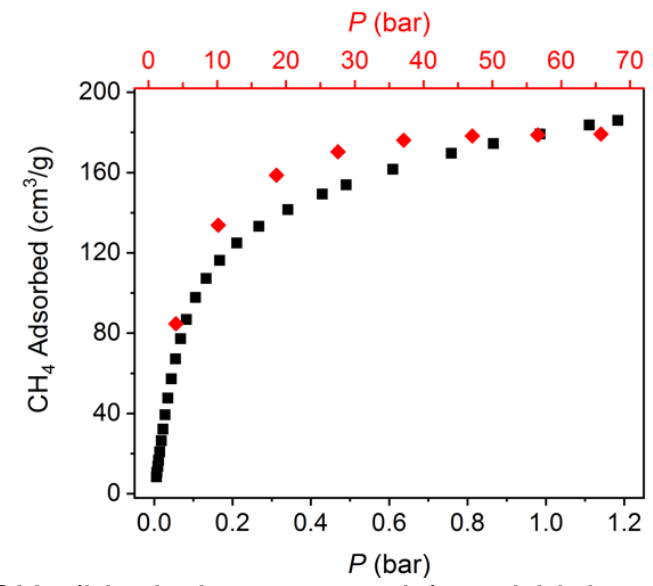

Figure S45. Low-pressure $\mathrm{CH}_{4}$ (black, bottom x-axis) and high-pressure $\mathrm{CH}_{4}$ (red, top x-axis) adsorption isotherms at $195 \mathrm{~K}$ and $298 \mathrm{~K}$ respectively, for Co-MOF-74.

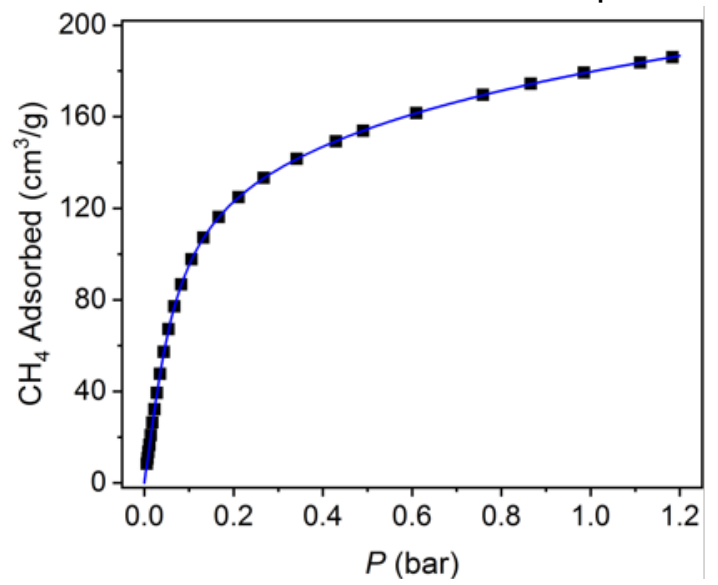

\begin{tabular}{|c|c|}
\hline $\mathrm{q}_{\mathrm{sat}, 1}$ & 93.573 \\
$\mathrm{~b}_{1}$ & 80.805 \\
\hline $\mathrm{v}_{1}$ & 1.502 \\
\hline $\mathrm{q}_{\mathrm{sat}, 2}$ & 258.963 \\
\hline $\mathrm{b}_{2}$ & 0.508 \\
\hline $\mathrm{v}_{2}$ & 0.623 \\
\hline $\mathrm{q}_{\mathrm{sat}, 3}$ & 0 \\
\hline $\mathrm{b}_{3}$ & 0 \\
\hline $\mathrm{v}_{3}$ & 0 \\
\hline
\end{tabular}

Figure S46. Low pressure $\mathrm{CH}_{4}$ isotherm with fit (left) and corresponding fitting parameters (right) for Co-MOF-74at $195 \mathrm{~K}$.

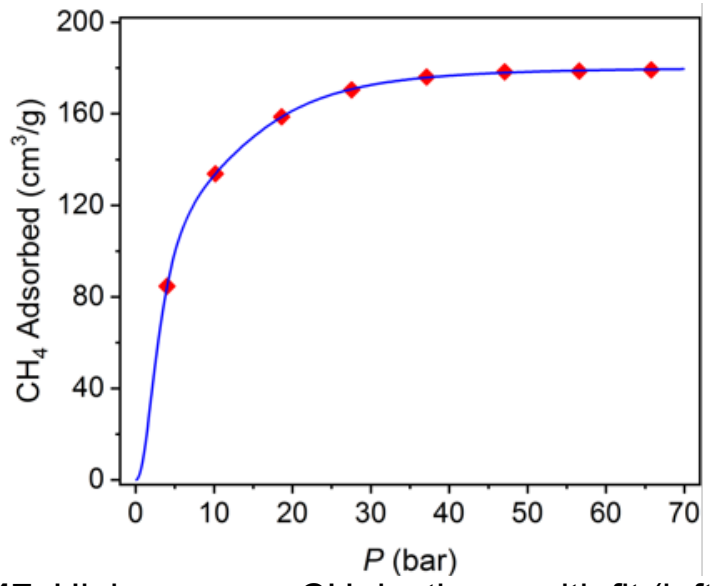

\begin{tabular}{|c|c|}
\hline $\mathrm{q}_{\mathrm{sat}, 1}$ & 43.801 \\
$\mathrm{~b}_{1}$ & $2.260 \mathrm{e}-4$ \\
\hline $\mathrm{v}_{1}$ & 2.975 \\
\hline $\mathrm{q}_{\mathrm{sat}, 2}$ & 136.534 \\
\hline $\mathrm{b}_{2}$ & 0.083 \\
\hline $\mathrm{v}_{2}$ & 2.129 \\
\hline $\mathrm{q}_{\mathrm{sat}, 3}$ & 0 \\
\hline $\mathrm{b}_{3}$ & 0 \\
\hline $\mathrm{v}_{3}$ & 0 \\
\hline
\end{tabular}

Figure S47. High pressure $\mathrm{CH}_{4}$ isotherm with fit (left) and corresponding fitting parameters (right) for Co-MOF-74 at $298 \mathrm{~K}$. 


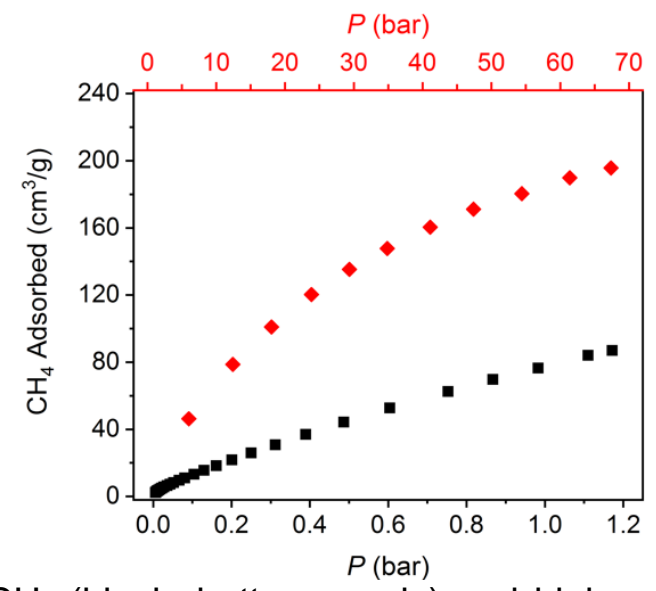

Figure S48. Low-pressure $\mathrm{CH}_{4}$ (black, bottom x-axis) and high-pressure $\mathrm{CH}_{4}$ (red, top x-axis) adsorption isotherms at $195 \mathrm{~K}$ and $298 \mathrm{~K}$ respectively, for Cr-MIL-100.

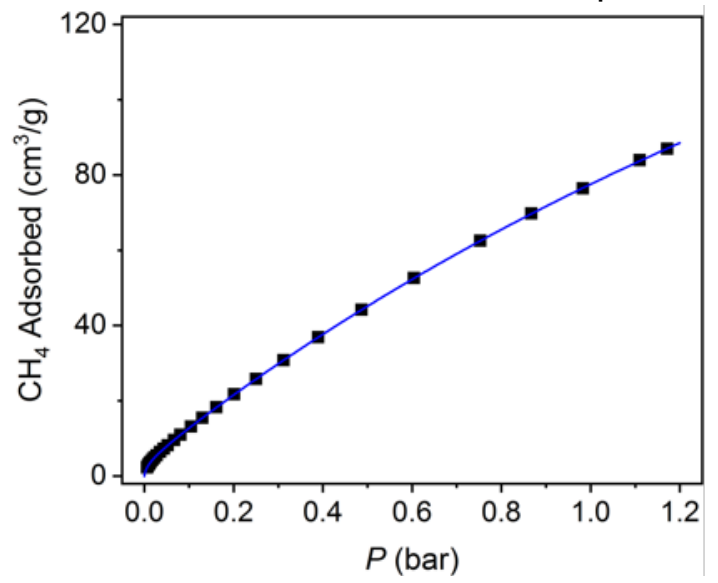

\begin{tabular}{|c|c|}
\hline $\mathrm{q}_{\mathrm{sat}, 1}$ & 303.251 \\
$\mathrm{~b}_{1}$ & 0.312 \\
\hline $\mathrm{v}_{1}$ & 1.040 \\
\hline $\mathrm{q}_{\mathrm{sat}, 2}$ & 5.708 \\
\hline $\mathrm{b}_{2}$ & 15.829 \\
\hline $\mathrm{v}_{2}$ & 0.692 \\
\hline $\mathrm{q}_{\mathrm{sat}, 3}$ & 0 \\
\hline $\mathrm{b}_{3}$ & 0 \\
\hline $\mathrm{v}_{3}$ & 0 \\
\hline
\end{tabular}

Figure S49. Low pressure $\mathrm{CH}_{4}$ isotherm with fit (left) and corresponding fitting parameters (right) for Cr-MIL-100 at $195 \mathrm{~K}$.

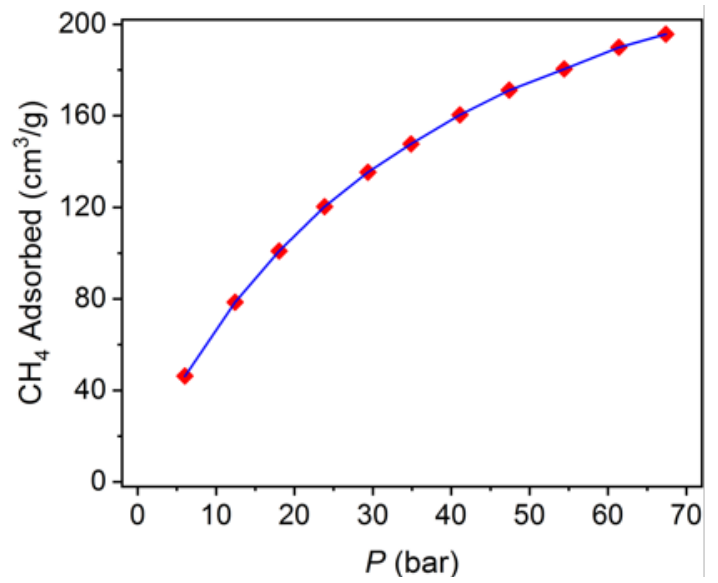

\begin{tabular}{|c|c|}
\hline $\mathrm{q}_{\mathrm{sat}, 1}$ & 304.733 \\
$\mathrm{~b}_{1}$ & 0.029 \\
\hline $\mathrm{v}_{1}$ & 0.979 \\
\hline $\mathrm{q}_{\mathrm{sat}, 2}$ & 0 \\
\hline $\mathrm{b}_{2}$ & 0 \\
\hline $\mathrm{v}_{2}$ & 0 \\
\hline $\mathrm{q}_{\mathrm{sat}, 3}$ & 0 \\
\hline $\mathrm{b}_{3}$ & 0 \\
\hline $\mathrm{v}_{3}$ & 0 \\
\hline
\end{tabular}

Figure S50. High pressure $\mathrm{CH}_{4}$ isotherm with fit (left) and corresponding fitting parameters (right) for Cr-MIL-100 at $298 \mathrm{~K}$. 


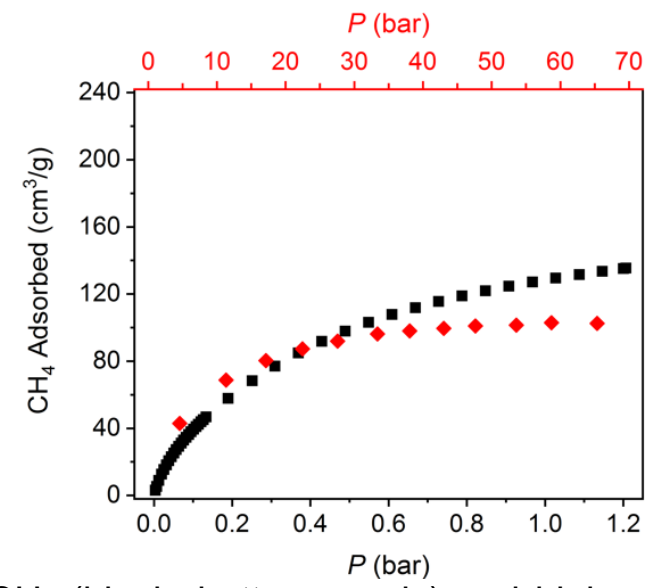

Figure S51. Low-pressure $\mathrm{CH}_{4}$ (black, bottom x-axis) and high-pressure $\mathrm{CH}_{4}$ (red, top x-axis) adsorption isotherms at $195 \mathrm{~K}$ and $298 \mathrm{~K}$ respectively, for $\mathrm{Cr}_{24}\left({ }^{\mathrm{t}} \mathrm{Bu}-\mathrm{bdc}\right)_{24}$.

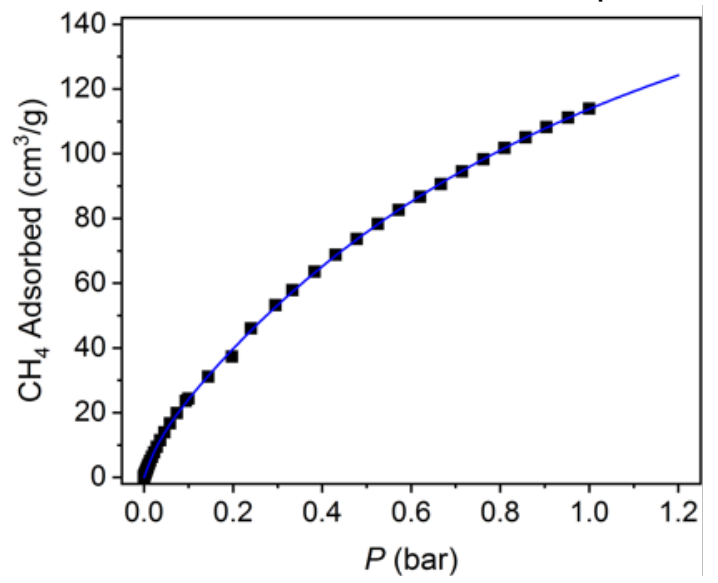

\begin{tabular}{|c|c|}
\hline $\mathrm{q}_{\mathrm{sat}, 1}$ & 132.438 \\
\hline $\mathrm{b}_{1}$ & 2.312 \\
\hline $\mathrm{v}_{1}$ & 1.290 \\
\hline $\mathrm{q}_{\mathrm{sat}, 2}$ & 38.010 \\
\hline $\mathrm{b}_{2}$ & 17.868 \\
\hline $\mathrm{v}_{2}$ & 0.972 \\
\hline $\mathrm{q}_{\mathrm{sat}, 3}$ & 0 \\
\hline $\mathrm{b}_{3}$ & 0 \\
\hline $\mathrm{v}_{3}$ & 0 \\
\hline
\end{tabular}

Figure S52. Low pressure $\mathrm{CH}_{4}$ isotherm with fit (left) and corresponding fitting parameters (right) for $\mathrm{Cr}_{24}\left({ }^{\mathrm{t} B u}-\mathrm{bdc}\right)_{24}$ at $195 \mathrm{~K}$.

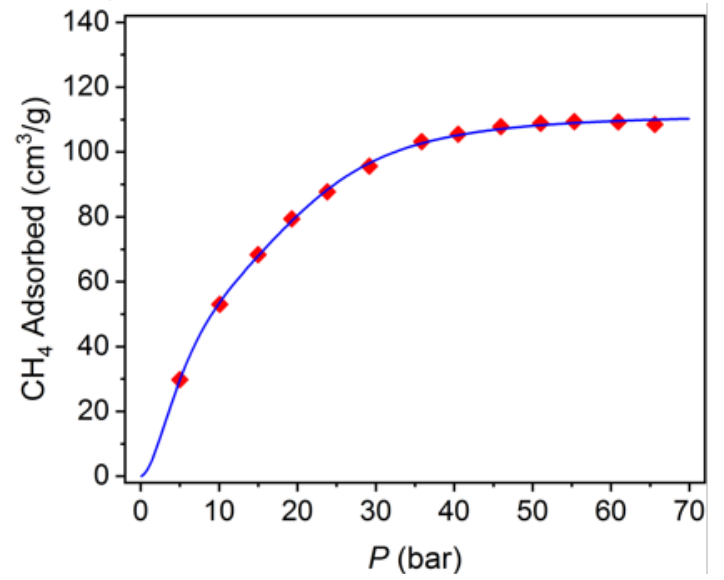

\begin{tabular}{|c|c|}
\hline $\mathrm{q}_{\mathrm{sat}, 1}$ & 28.796 \\
$\mathrm{~b}_{1}$ & $4.201 \mathrm{e}-4$ \\
\hline $\mathrm{v}_{1}$ & 2.560 \\
\hline $\mathrm{q}_{\mathrm{sat}, 2}$ & 76.656 \\
\hline $\mathrm{b}_{2}$ & 0.119 \\
\hline $\mathrm{v}_{2}$ & 1.53564 \\
\hline $\mathrm{q}_{\mathrm{sat}, 3}$ & 0 \\
\hline $\mathrm{b}_{3}$ & 0 \\
\hline $\mathrm{v}_{3}$ & 0 \\
\hline
\end{tabular}

Figure S53. High pressure $\mathrm{CH}_{4}$ isotherm with fit (left) and corresponding fitting parameters (right) for $\mathrm{Cr}_{24}\left({ }^{\mathrm{t}} \mathrm{Bu}-\mathrm{bdc}\right)_{24}$ at $298 \mathrm{~K}$. 


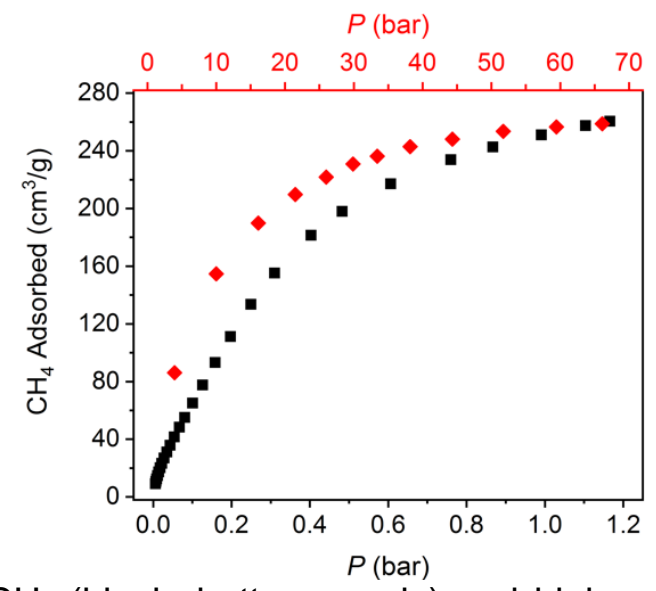

Figure S54. Low-pressure $\mathrm{CH}_{4}$ (black, bottom x-axis) and high-pressure $\mathrm{CH}_{4}$ (red, top x-axis) adsorption isotherms at $195 \mathrm{~K}$ and $298 \mathrm{~K}$ respectively, for $\mathrm{Cu}_{2}$ (adip).

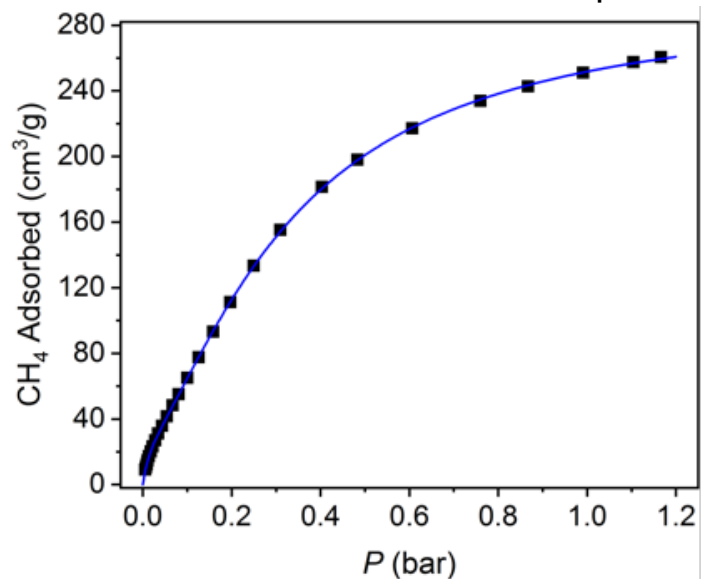

\begin{tabular}{|c|c|}
\hline $\mathrm{q}_{\mathrm{sat}, 1}$ & 265.023 \\
$\mathrm{~b}_{1}$ & 5.144 \\
\hline $\mathrm{v}_{1}$ & 1.494 \\
\hline $\mathrm{q}_{\mathrm{sat}, 2}$ & 30.104 \\
\hline $\mathrm{b}_{2}$ & 125.393 \\
\hline $\mathrm{v}_{2}$ & 1.138 \\
\hline $\mathrm{q}_{\mathrm{sat}, 3}$ & 0 \\
\hline $\mathrm{b}_{3}$ & 0 \\
\hline $\mathrm{v}_{3}$ & 0 \\
\hline
\end{tabular}

Figure S55. Low pressure $\mathrm{CH}_{4}$ isotherm with fit (left) and corresponding fitting parameters (right) for $\mathrm{Cu}_{2}$ (adip) at $195 \mathrm{~K}$.

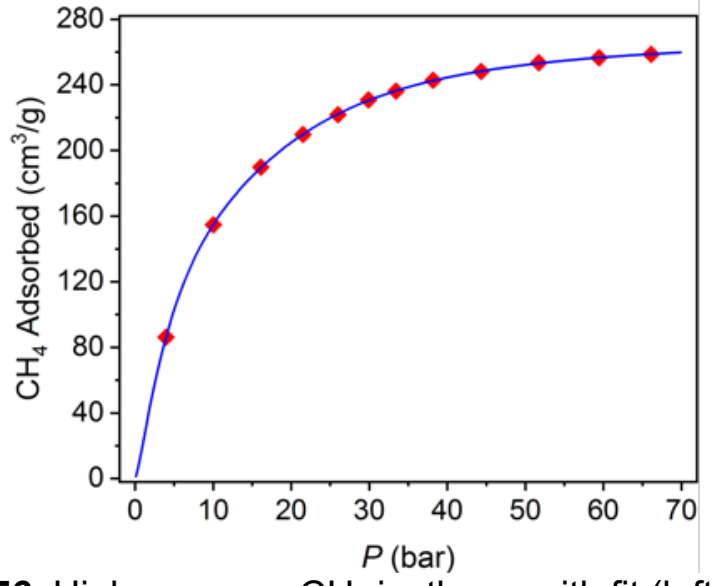

\begin{tabular}{|c|c|}
\hline $\mathrm{q}_{\mathrm{sat}, 1}$ & 26.812 \\
$\mathrm{~b}_{1}$ & 0.106 \\
\hline $\mathrm{v}_{1}$ & 1.272 \\
\hline $\mathrm{q}_{\mathrm{sat}, 2}$ & 44.588 \\
\hline $\mathrm{b}_{2}$ & $2.084 \mathrm{e}-4$ \\
\hline $\mathrm{v}_{2}$ & 2.677 \\
\hline $\mathrm{q}_{\mathrm{sat}, 3}$ & 0 \\
\hline $\mathrm{b}_{3}$ & 0 \\
\hline $\mathrm{v}_{3}$ & 0 \\
\hline
\end{tabular}

Figure S56. High pressure $\mathrm{CH}_{4}$ isotherm with fit (left) and corresponding fitting parameters (right) for $\mathrm{Cu}_{2}$ (adip) at $298 \mathrm{~K}$. 


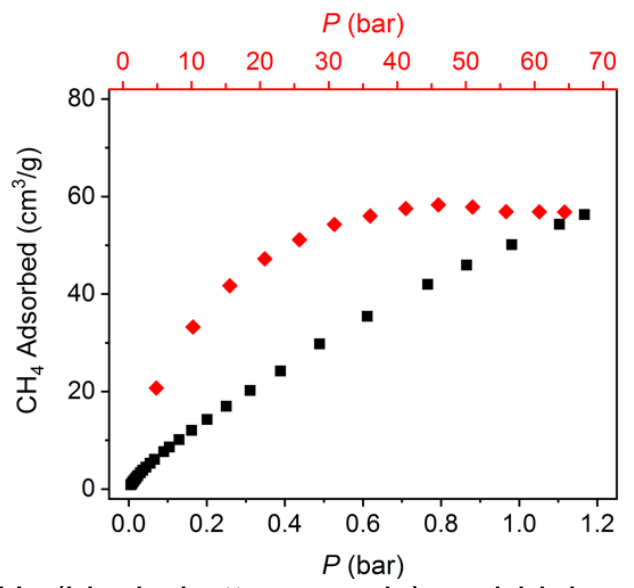

Figure S57. Low-pressure $\mathrm{CH}_{4}$ (black, bottom x-axis) and high-pressure $\mathrm{CH}_{4}$ (red, top x-axis) adsorption isotherms at $195 \mathrm{~K}$ and $298 \mathrm{~K}$ respectively, for DUT-8 (Zn).

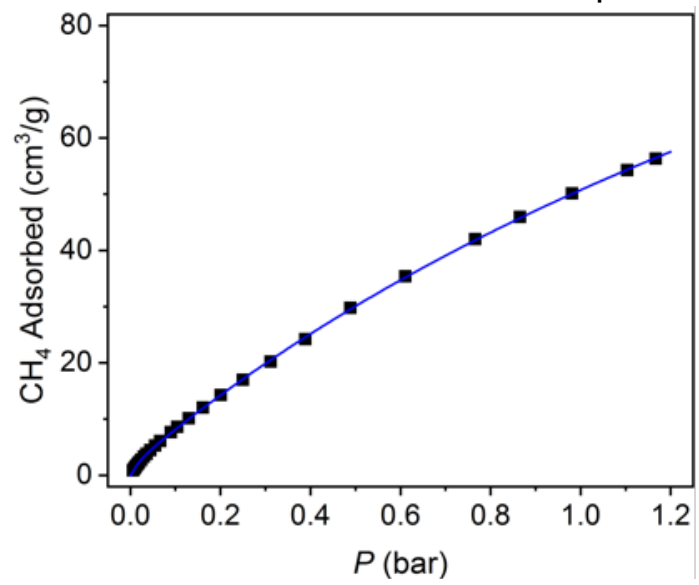

\begin{tabular}{|c|c|}
\hline $\mathrm{q}_{\mathrm{sat}, 1}$ & 1.808 \\
$\mathrm{~b}_{1}$ & 668.169 \\
\hline $\mathrm{v}_{1}$ & 1.572 \\
\hline $\mathrm{q}_{\mathrm{sat}, 2}$ & 181.684 \\
\hline $\mathrm{b}_{2}$ & 0.36855 \\
\hline $\mathrm{v}_{2}$ & 1 \\
\hline $\mathrm{q}_{\mathrm{sat}, 3}$ & 0 \\
\hline $\mathrm{b}_{3}$ & 0 \\
\hline $\mathrm{v}_{3}$ & 0 \\
\hline
\end{tabular}

Figure S58. Low pressure $\mathrm{CH}_{4}$ isotherm with fit (left) and corresponding fitting parameters (right) for DUT-8 (Zn) at $195 \mathrm{~K}$.

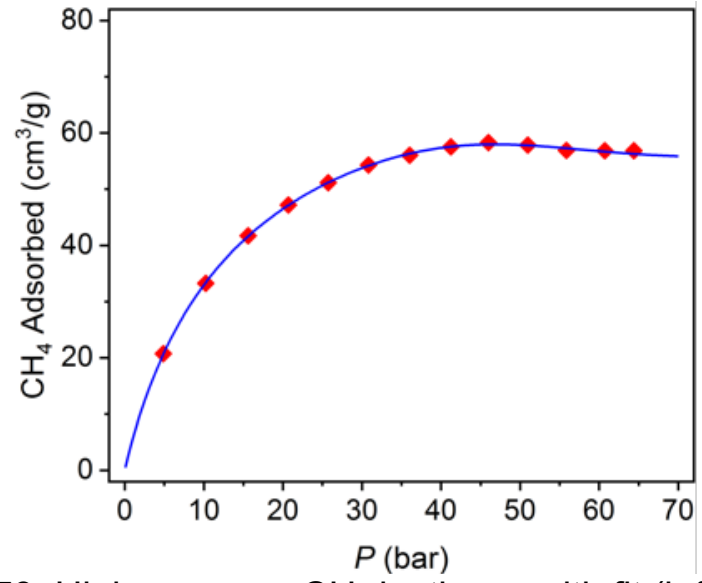

\begin{tabular}{|c|c|}
\hline $\mathrm{q}_{\mathrm{sat}, 1}$ & 82.392 \\
$\mathrm{~b}_{1}$ & 0.073 \\
\hline $\mathrm{v}_{1}$ & 0.960 \\
\hline $\mathrm{q}_{\mathrm{sat}, 2}$ & -14.719 \\
\hline $\mathrm{b}_{2}$ & $1.16 \mathrm{e}-10$ \\
\hline $\mathrm{v}_{2}$ & 5.643 \\
\hline $\mathrm{q}_{\mathrm{sat}, 3}$ & 0 \\
\hline $\mathrm{b}_{3}$ & 0 \\
\hline $\mathrm{v}_{3}$ & 0 \\
\hline
\end{tabular}

Figure S59. High pressure $\mathrm{CH}_{4}$ isotherm with fit (left) and corresponding fitting parameters (right) for DUT-8 (Zn) at $298 \mathrm{~K}$. 


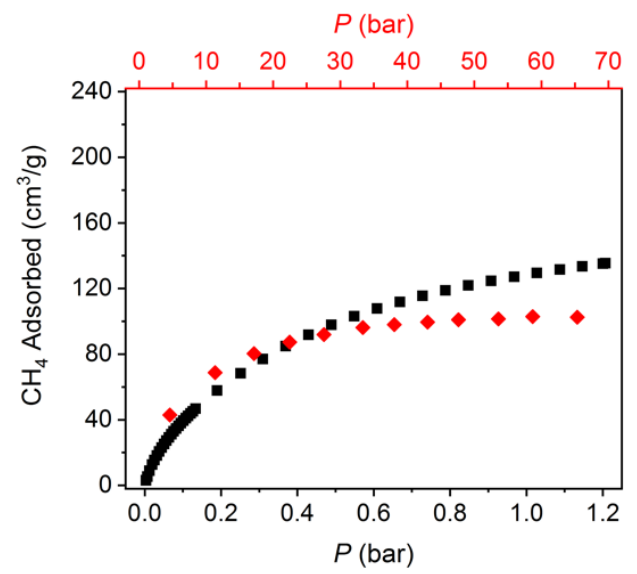

Figure S60. Low-pressure $\mathrm{CH}_{4}$ (black, bottom x-axis) and high-pressure $\mathrm{CH}_{4}$ (red, top x-axis) adsorption isotherms at $195 \mathrm{~K}$ and $298 \mathrm{~K}$ respectively, for $\mathrm{Fe}_{24}(\mathrm{Me}-\mathrm{bdc})_{24}(\mathrm{dabco})_{6}$.

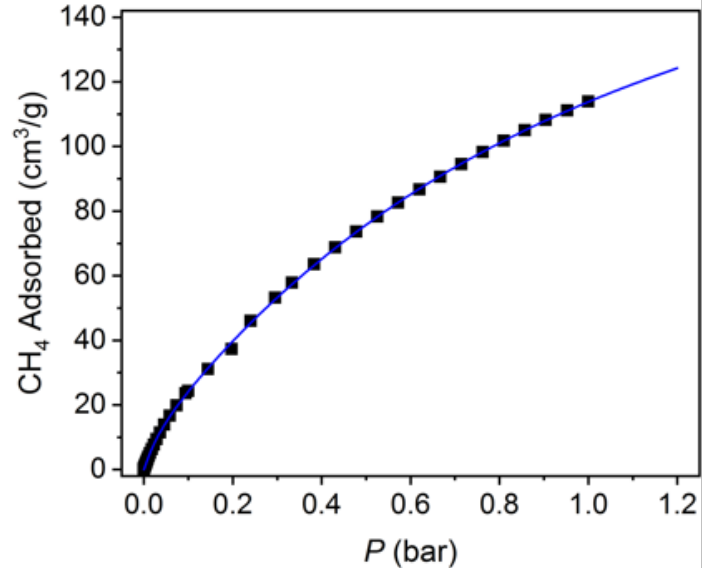

\begin{tabular}{|c|c|}
\hline $\mathrm{q}_{\mathrm{sat}, 1}$ & 132.438 \\
\hline $\mathrm{b}_{1}$ & 2.312 \\
\hline $\mathrm{v}_{1}$ & 1.290 \\
\hline $\mathrm{q}_{\mathrm{sat}, 2}$ & 38.010 \\
\hline $\mathrm{b}_{2}$ & 17.868 \\
\hline $\mathrm{v}_{2}$ & 0.972 \\
\hline $\mathrm{q}_{\mathrm{sat}, 3}$ & 0 \\
\hline $\mathrm{b}_{3}$ & 0 \\
\hline $\mathrm{v}_{3}$ & 0 \\
\hline
\end{tabular}

Figure S61. Low pressure $\mathrm{CH}_{4}$ isotherm with fit (left) and corresponding fitting parameters (right) for $\mathrm{Fe}_{24}(\mathrm{Me}-\mathrm{bdc})_{24}(\text { dabco })_{6}$ at $195 \mathrm{~K}$.

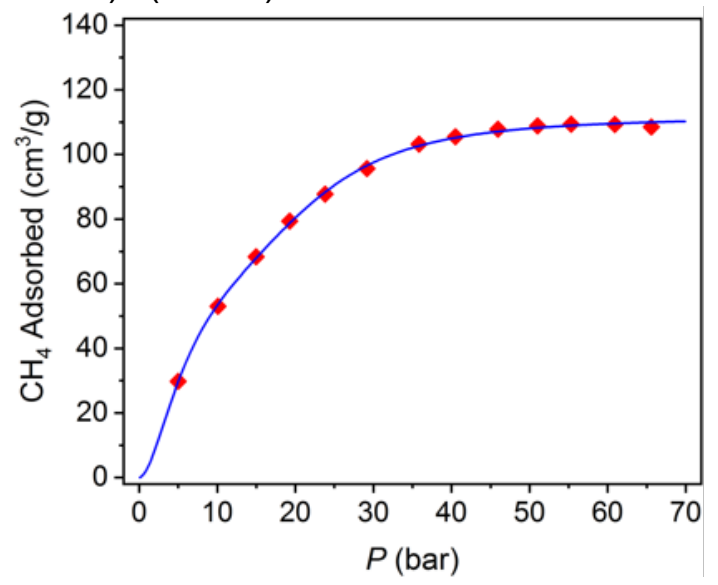

\begin{tabular}{|c|c|}
\hline $\mathrm{q}_{\mathrm{sat}, 1}$ & 28.796 \\
$\mathrm{~b}_{1}$ & $4.201 \mathrm{e}-4$ \\
\hline $\mathrm{v}_{1}$ & 2.560 \\
\hline $\mathrm{q}_{\mathrm{sat}, 2}$ & 76.656 \\
\hline $\mathrm{b}_{2}$ & 0.119 \\
\hline $\mathrm{v}_{2}$ & 1.53564 \\
\hline $\mathrm{q}_{\mathrm{sat}, 3}$ & 0 \\
\hline $\mathrm{b}_{3}$ & 0 \\
\hline $\mathrm{v}_{3}$ & 0 \\
\hline
\end{tabular}

Figure S62. High pressure $\mathrm{CH}_{4}$ isotherm with fit (left) and corresponding fitting parameters (right) for $\mathrm{Fe}_{24}(\mathrm{Me}-\mathrm{bdc})_{24}(\mathrm{dabco})_{6}$ at $298 \mathrm{~K}$. 


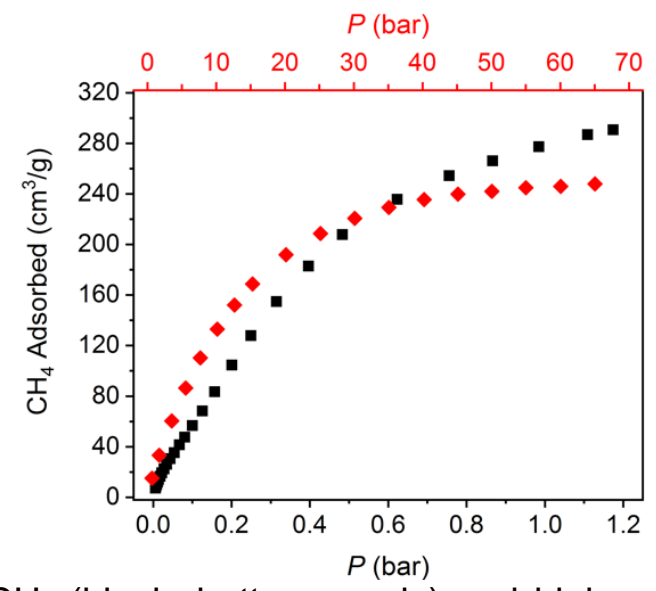

Figure S63. Low-pressure $\mathrm{CH}_{4}$ (black, bottom x-axis) and high-pressure $\mathrm{CH}_{4}$ (red, top x-axis) adsorption isotherms at $195 \mathrm{~K}$ and $298 \mathrm{~K}$ respectively, for HKUST-1.

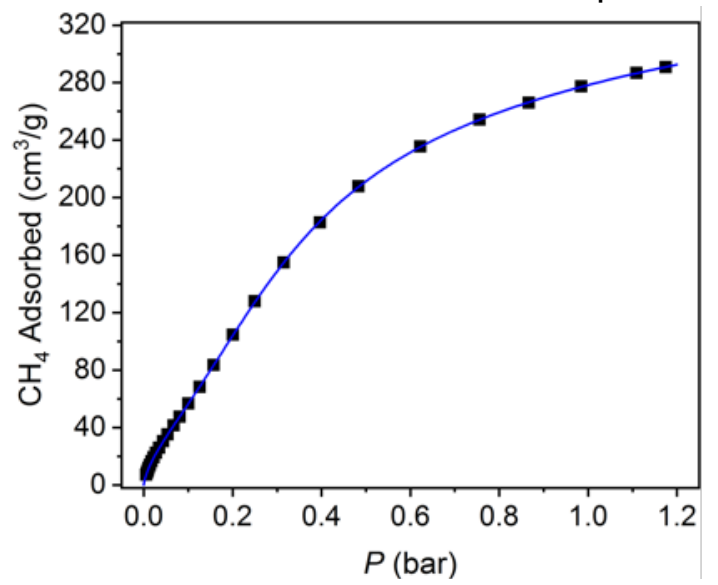

\begin{tabular}{|c|c|}
\hline $\mathrm{q}_{\mathrm{sat}, 1}$ & 352.834 \\
$\mathrm{~b}_{1}$ & 0.829 \\
\hline $\mathrm{v}_{1}$ & 0.708 \\
\hline $\mathrm{q}_{\mathrm{sat}, 2}$ & 126.908 \\
\hline $\mathrm{b}_{2}$ & 13.902 \\
\hline $\mathrm{v}_{2}$ & 2.364 \\
\hline $\mathrm{q}_{\mathrm{sat}, 3}$ & 0 \\
\hline $\mathrm{b}_{3}$ & 0 \\
\hline $\mathrm{v}_{3}$ & 0 \\
\hline
\end{tabular}

Figure S64. Low pressure $\mathrm{CH}_{4}$ isotherm with fit (left) and corresponding fitting parameters (right) for HKUST-1 at $195 \mathrm{~K}$.

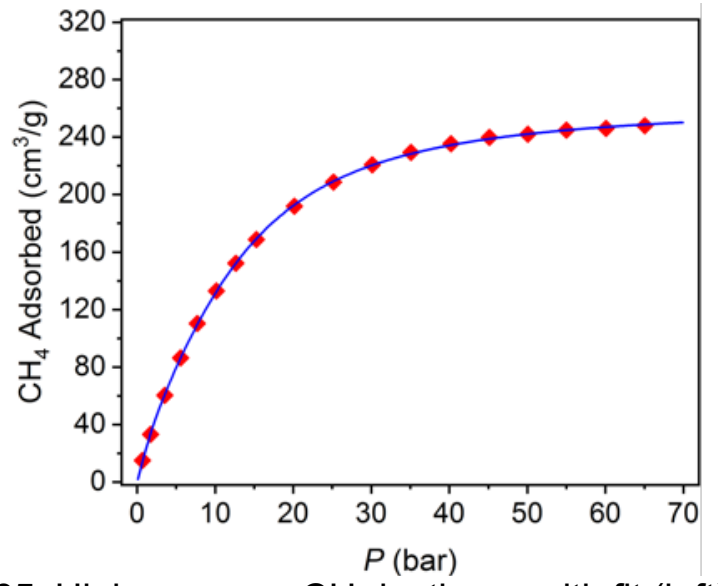

\begin{tabular}{|c|c|}
\hline $\mathrm{q}_{\mathrm{sat}, 1}$ & 115.933 \\
\hline $\mathrm{b}_{1}$ & 0.203 \\
\hline $\mathrm{v}_{1}$ & 1.069 \\
\hline $\mathrm{q}_{\mathrm{sat}, 2}$ & 147.353 \\
\hline $\mathrm{b}_{2}$ & 0.007 \\
\hline $\mathrm{v}_{2}$ & 1.870 \\
\hline $\mathrm{q}_{\mathrm{sat}, 3}$ & 0 \\
\hline $\mathrm{b}_{3}$ & 0 \\
\hline $\mathrm{v}_{3}$ & 0 \\
\hline
\end{tabular}

Figure S65. High pressure $\mathrm{CH}_{4}$ isotherm with fit (left) and corresponding fitting parameters (right) for HKUST-1 at $298 \mathrm{~K}$. 


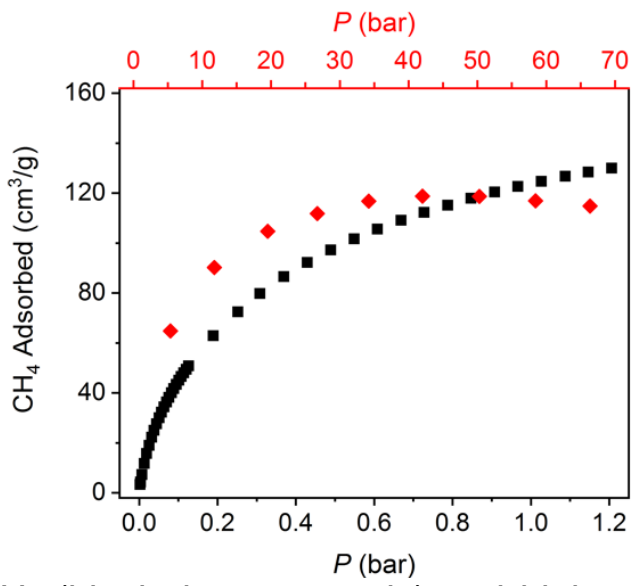

Figure S66. Low-pressure $\mathrm{CH}_{4}$ (black, bottom x-axis) and high-pressure $\mathrm{CH}_{4}$ (red, top x-axis) adsorption isotherms at $195 \mathrm{~K}$ and $298 \mathrm{~K}$ respectively, for $\mathrm{Mo}_{24}\left(\text { bdc- }-\mathrm{NH}_{2}\right)_{24}$.

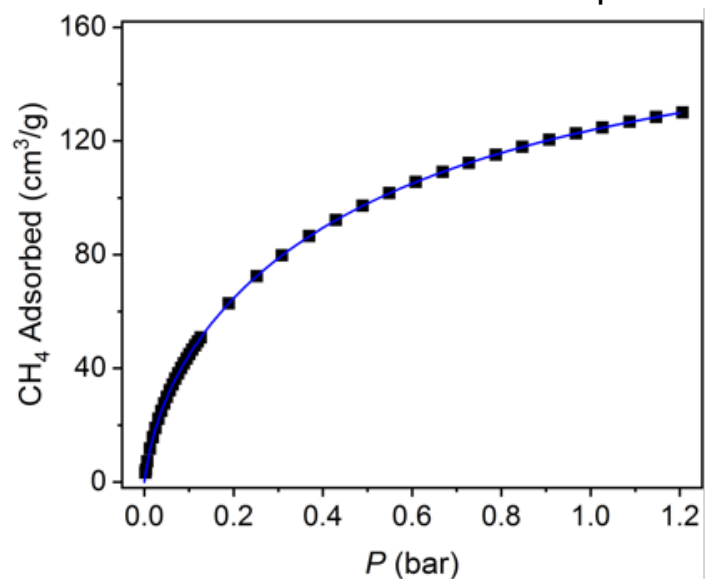

\begin{tabular}{|c|c|}
\hline $\mathrm{q}_{\mathrm{sat}, 1}$ & 32.909 \\
$\mathrm{~b}_{1}$ & 17.452 \\
\hline $\mathrm{v}_{1}$ & 0.909 \\
\hline $\mathrm{q}_{\mathrm{sat}, 2}$ & 144.670 \\
\hline $\mathrm{b}_{2}$ & 1.779 \\
\hline $\mathrm{v}_{2}$ & 0.993 \\
\hline $\mathrm{q}_{\mathrm{sat}, 3}$ & 0 \\
\hline $\mathrm{b}_{3}$ & 0 \\
\hline $\mathrm{v}_{3}$ & 0 \\
\hline
\end{tabular}

Figure S67. Low pressure $\mathrm{CH}_{4}$ isotherm with fit (left) and corresponding fitting parameters (right) for $\mathrm{Mo}_{24}\left(\mathrm{bdc}-\mathrm{NH}_{2}\right)_{24}$ at $195 \mathrm{~K}$.

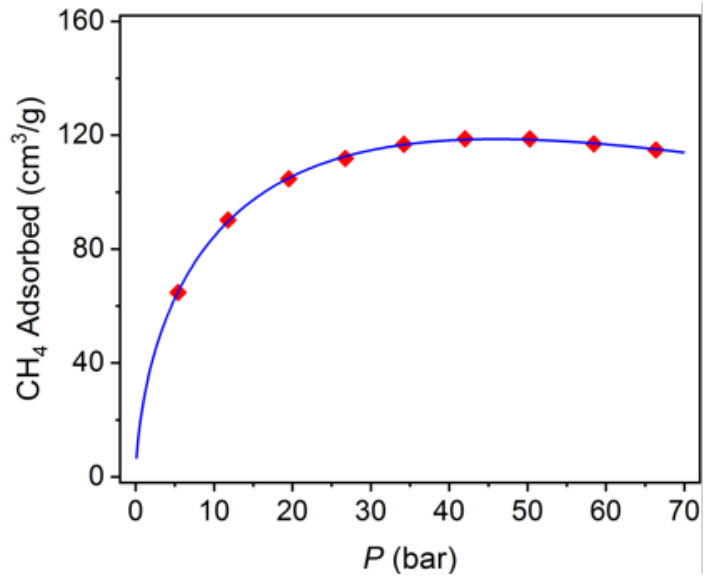

\begin{tabular}{|c|c|}
\hline $\mathrm{q}_{\mathrm{sat}, 1}$ & 370.716 \\
\hline $\mathrm{b}_{1}$ & 0.081 \\
\hline $\mathrm{v}_{1}$ & 0.641 \\
\hline $\mathrm{q}_{\mathrm{sat}, 2}$ & -500 \\
\hline $\mathrm{b}_{2}$ & 0.002 \\
\hline $\mathrm{v}_{2}$ & 1.104 \\
\hline $\mathrm{q}_{\mathrm{sat}, 3}$ & 0 \\
\hline $\mathrm{b}_{3}$ & 0 \\
\hline $\mathrm{v}_{3}$ & 0 \\
\hline
\end{tabular}

Figure S68. High pressure $\mathrm{CH}_{4}$ isotherm with fit (left) and corresponding fitting parameters (right) for $\mathrm{Mo}_{24}\left(\text { bdc- } \mathrm{NH}_{2}\right)_{24}$ at $298 \mathrm{~K}$. 


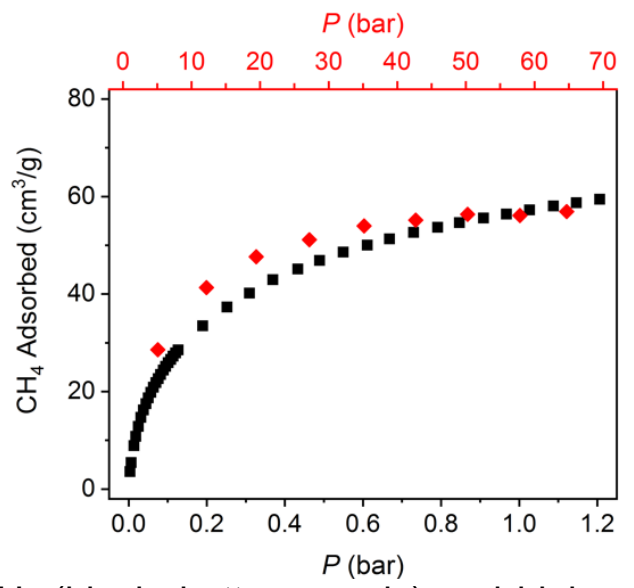

Figure S69. Low-pressure $\mathrm{CH}_{4}$ (black, bottom x-axis) and high-pressure $\mathrm{CH}_{4}$ (red, top x-axis) adsorption isotherms at $195 \mathrm{~K}$ and $298 \mathrm{~K}$ respectively, for $\mathrm{Mo}_{24}(\mathrm{bdc}-\mathrm{OH})_{24}$.

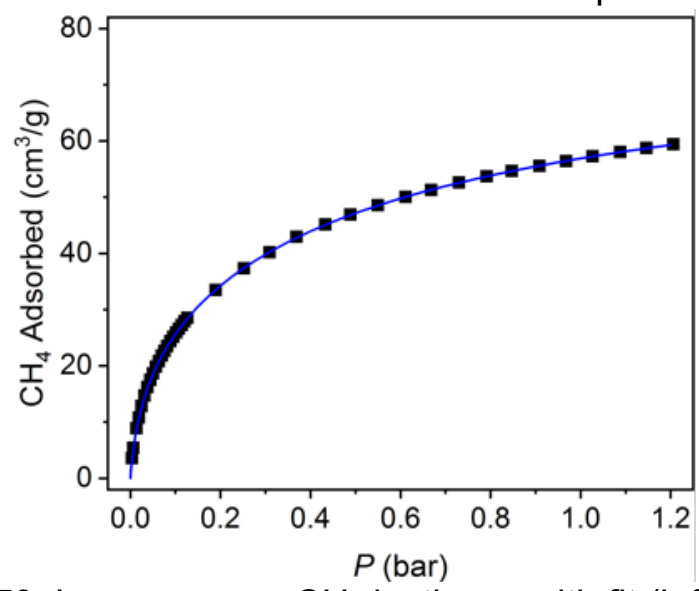

\begin{tabular}{|c|c|}
\hline $\mathrm{q}_{\mathrm{sat}, 1}$ & 12.084 \\
$\mathrm{~b}_{1}$ & 35.841 \\
\hline $\mathrm{v}_{1}$ & 0.947 \\
\hline $\mathrm{q}_{\mathrm{sat}, 2}$ & 72.742 \\
\hline $\mathrm{b}_{2}$ & 1.634 \\
\hline $\mathrm{v}_{2}$ & 0.764 \\
\hline $\mathrm{q}_{\mathrm{sat}, 3}$ & 0 \\
\hline $\mathrm{b}_{3}$ & 0 \\
\hline $\mathrm{v}_{3}$ & 0 \\
\hline
\end{tabular}

Figure S70. Low pressure $\mathrm{CH}_{4}$ isotherm with fit (left) and corresponding fitting parameters (right) for $\mathrm{Mo}_{24}(\mathrm{bdc}-\mathrm{OH}) 24$ at $195 \mathrm{~K}$.

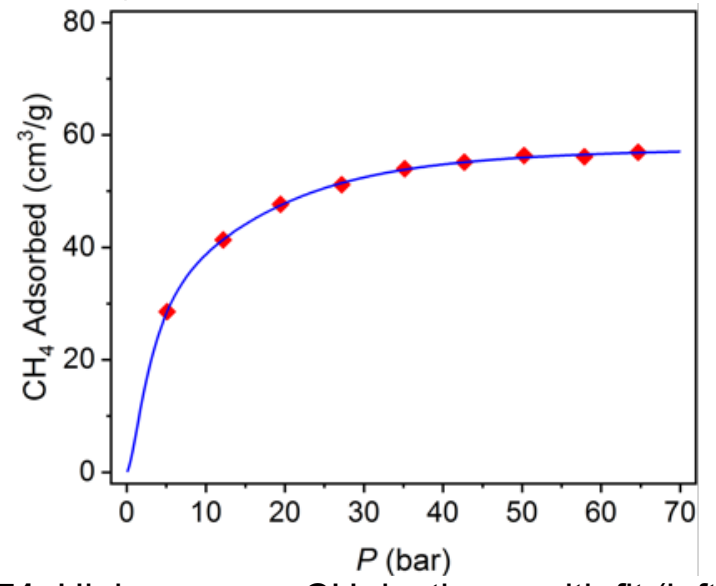

\begin{tabular}{|c|c|}
\hline $\mathrm{q}_{\mathrm{sat}, 1}$ & 12.582 \\
\hline $\mathrm{b}_{1}$ & $3.58 \mathrm{e}-4$ \\
\hline $\mathrm{v}_{1}$ & 2.576 \\
\hline $\mathrm{q}_{\mathrm{sat}, 2}$ & 45.601 \\
\hline $\mathrm{b}_{2}$ & 0.144 \\
\hline $\mathrm{v}_{2}$ & 1.488 \\
\hline $\mathrm{q}_{\mathrm{sat}, 3}$ & 0 \\
\hline $\mathrm{b}_{3}$ & 0 \\
\hline $\mathrm{v}_{3}$ & 0 \\
\hline
\end{tabular}

Figure S71. High pressure $\mathrm{CH}_{4}$ isotherm with fit (left) and corresponding fitting parameters (right) for $\mathrm{Mo}_{24}(\mathrm{bdc}-\mathrm{OH})_{24}$ at $298 \mathrm{~K}$. 


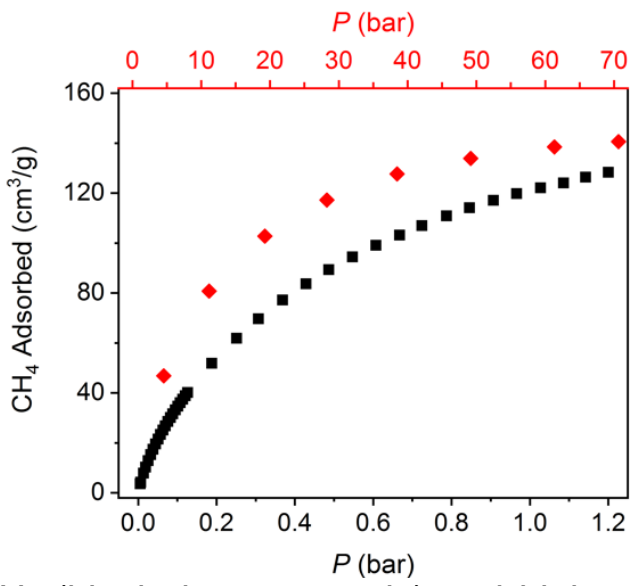

Figure S72. Low-pressure $\mathrm{CH}_{4}$ (black, bottom x-axis) and high-pressure $\mathrm{CH}_{4}$ (red, top x-axis) adsorption isotherms at $195 \mathrm{~K}$ and $298 \mathrm{~K}$ respectively, for $\mathrm{Mo}_{24}(\text { bdc-tBu})_{24}$.

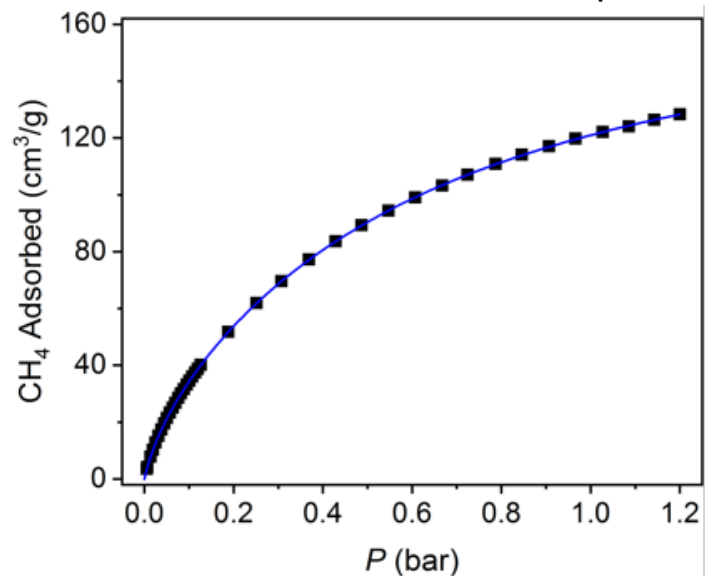

\begin{tabular}{|c|c|}
\hline $\mathrm{q}_{\mathrm{sat}, 1}$ & 145.979 \\
\hline $\mathrm{b}_{1}$ & 1.685 \\
\hline $\mathrm{v}_{1}$ & 1.152 \\
\hline $\mathrm{q}_{\mathrm{sat}, 2}$ & 31.760 \\
\hline $\mathrm{b}_{2}$ & 12.118 \\
\hline $\mathrm{v}_{2}$ & 0.923 \\
\hline $\mathrm{q}_{\mathrm{sat}, 3}$ & 0 \\
\hline $\mathrm{b}_{3}$ & 0 \\
\hline $\mathrm{v}_{3}$ & 0 \\
\hline
\end{tabular}

Figure S73. Low pressure $\mathrm{CH}_{4}$ isotherm with fit (left) and corresponding fitting parameters (right) for $\mathrm{Mo}_{24}(\mathrm{bdc}-\mathrm{tBu})_{24}$ at $195 \mathrm{~K}$.

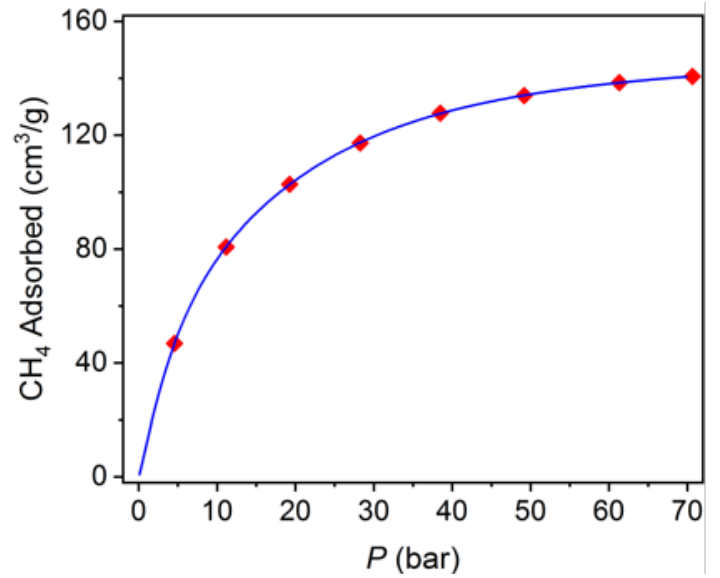

\begin{tabular}{|c|c|}
\hline $\mathrm{q}_{\mathrm{sat}, 1}$ & 124.346 \\
\hline $\mathrm{b}_{1}$ & 0.102 \\
\hline $\mathrm{v}_{1}$ & 1.158 \\
\hline $\mathrm{q}_{\mathrm{sat}, 2}$ & 27.173 \\
\hline $\mathrm{b}_{2}$ & $4.33 \mathrm{e}-4$ \\
\hline $\mathrm{v}_{2}$ & 2.340 \\
\hline $\mathrm{q}_{\mathrm{sat}, 3}$ & 0 \\
\hline $\mathrm{b}_{3}$ & 0 \\
\hline $\mathrm{v}_{3}$ & 0 \\
\hline
\end{tabular}

Figure S74. High pressure $\mathrm{CH}_{4}$ isotherm with fit (left) and corresponding fitting parameters (right) for $\mathrm{Mo}_{24}(\mathrm{bdc}-\mathrm{tBu})_{24}$ at $298 \mathrm{~K}$. 


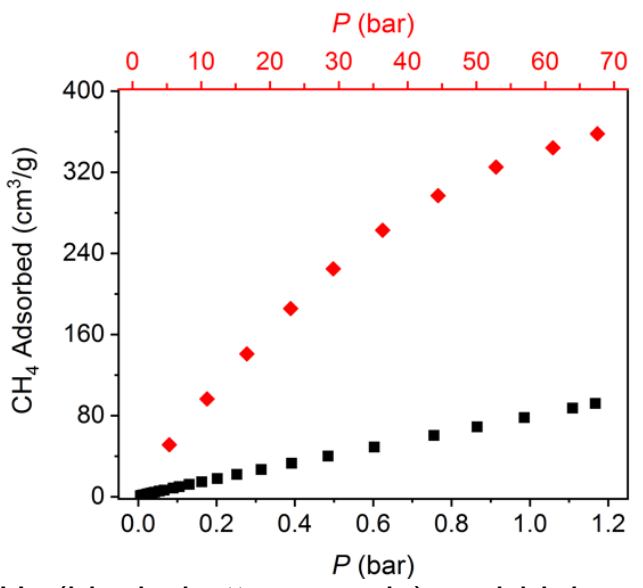

Figure S75. Low-pressure $\mathrm{CH}_{4}$ (black, bottom x-axis) and high-pressure $\mathrm{CH}_{4}$ (red, top x-axis) adsorption isotherms at $195 \mathrm{~K}$ and $298 \mathrm{~K}$ respectively, for MOF-177.

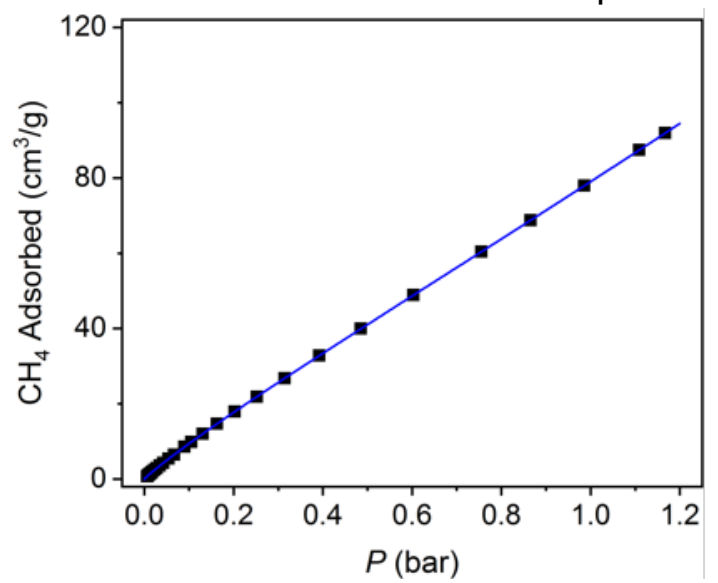

\begin{tabular}{|c|c|}
\hline $\mathrm{q}_{\mathrm{sat}, 1}$ & 344.830 \\
$\mathrm{~b}_{1}$ & 0.232 \\
\hline $\mathrm{v}_{1}$ & 0.923 \\
\hline $\mathrm{q}_{\mathrm{sat}, 2}$ & 420.432 \\
\hline $\mathrm{b}_{2}$ & 0.035 \\
\hline $\mathrm{v}_{2}$ & 2.051 \\
\hline $\mathrm{q}_{\mathrm{sat}, 3}$ & 0 \\
\hline $\mathrm{b}_{3}$ & 0 \\
\hline $\mathrm{v}_{3}$ & 0 \\
\hline
\end{tabular}

Figure S76. Low pressure $\mathrm{CH}_{4}$ isotherm with fit (left) and corresponding fitting parameters (right) for MOF-177 at $195 \mathrm{~K}$.

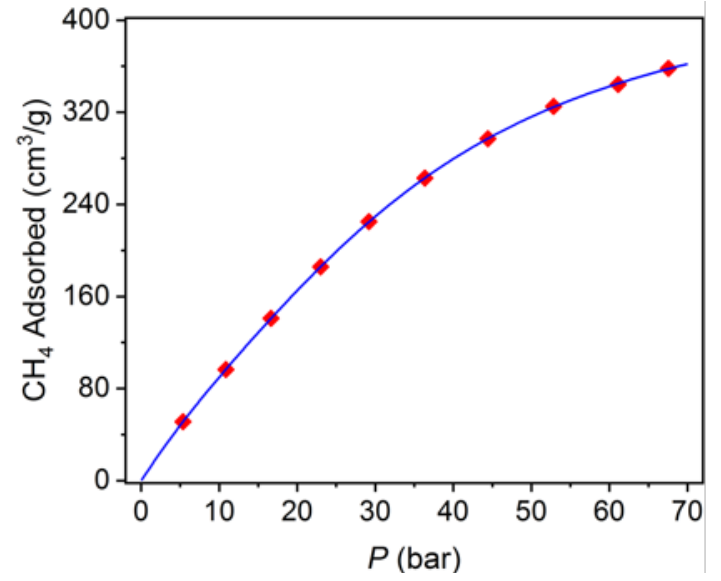

\begin{tabular}{|c|c|}
\hline $\mathrm{q}_{\mathrm{sat}, 1}$ & 280.546 \\
$\mathrm{~b}_{1}$ & 0.037 \\
\hline $\mathrm{v}_{1}$ & 1.035 \\
\hline $\mathrm{q}_{\mathrm{sat}, 2}$ & 185.024 \\
\hline $\mathrm{b}_{2}$ & $2.97 \mathrm{e}-4$ \\
\hline $\mathrm{v}_{2}$ & 2.266 \\
\hline $\mathrm{q}_{\mathrm{sat}, 3}$ & 0 \\
\hline $\mathrm{b}_{3}$ & 0 \\
\hline $\mathrm{v}_{3}$ & 0 \\
\hline
\end{tabular}

Figure S77. High pressure $\mathrm{CH}_{4}$ isotherm with fit (left) and corresponding fitting parameters (right) for MOF-177 at $298 \mathrm{~K}$. 


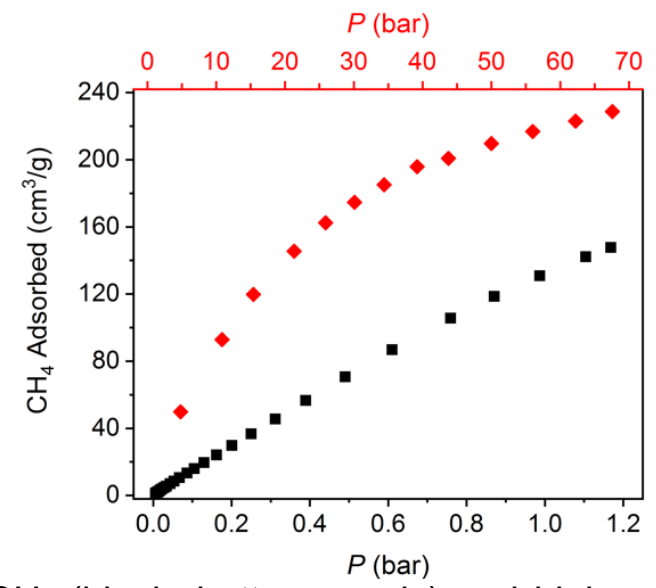

Figure S78. Low-pressure $\mathrm{CH}_{4}$ (black, bottom x-axis) and high-pressure $\mathrm{CH}_{4}$ (red, top x-axis) adsorption isotherms at $195 \mathrm{~K}$ and $298 \mathrm{~K}$ respectively, for Ni(ndc)ted 0.5 .

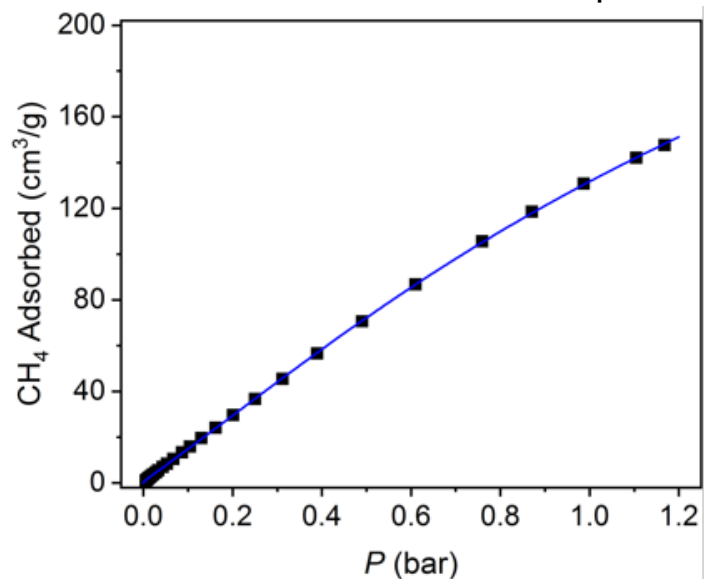

\begin{tabular}{|c|c|}
\hline $\mathrm{q}_{\mathrm{sat}, 1}$ & 500 \\
$\mathrm{~b}_{1}$ & 0.055 \\
\hline $\mathrm{v}_{1}$ & 0.627 \\
\hline $\mathrm{q}_{\mathrm{sat}, 2}$ & 300.846 \\
\hline $\mathrm{b}_{2}$ & 0.540 \\
\hline $\mathrm{v}_{2}$ & 1.274 \\
\hline $\mathrm{q}_{\mathrm{sat}, 3}$ & 0 \\
\hline $\mathrm{b}_{3}$ & 0 \\
\hline $\mathrm{v}_{3}$ & 0 \\
\hline
\end{tabular}

Figure S79. Low pressure $\mathrm{CH}_{4}$ isotherm with fit (left) and corresponding fitting parameters (right) for $\mathrm{Ni}(\mathrm{ndc}) \mathrm{ted}_{0.5}$ at $195 \mathrm{~K}$.

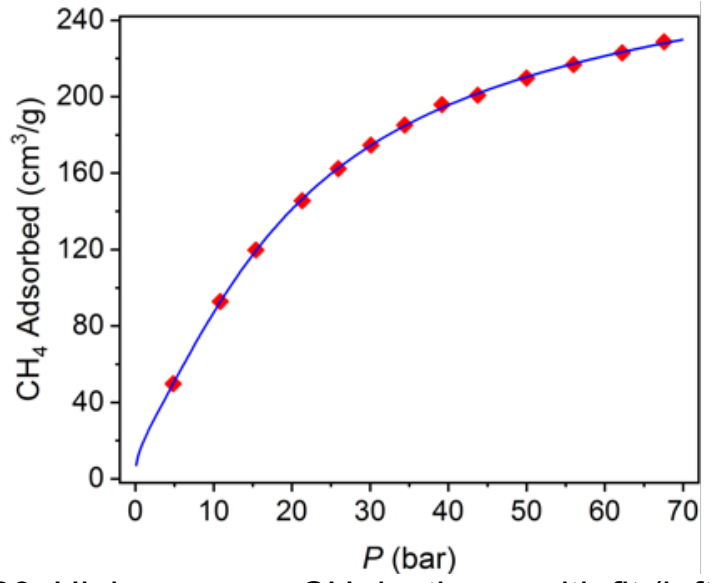

\begin{tabular}{|c|c|}
\hline $\mathrm{q}_{\mathrm{sat}, 1}$ & 499.998 \\
$\mathrm{~b}_{1}$ & 0.037 \\
\hline $\mathrm{v}_{1}$ & 0.412 \\
\hline $\mathrm{q}_{\mathrm{sat}, 2}$ & 157.784 \\
\hline $\mathrm{b}_{2}$ & 0.009 \\
\hline $\mathrm{v}_{2}$ & 1.605 \\
\hline $\mathrm{q}_{\mathrm{sat}, 3}$ & 0 \\
\hline $\mathrm{b}_{3}$ & 0 \\
\hline $\mathrm{v}_{3}$ & 0 \\
\hline
\end{tabular}

Figure S80. High pressure $\mathrm{CH}_{4}$ isotherm with fit (left) and corresponding fitting parameters (right) for $\mathrm{Ni}(\mathrm{ndc}) \mathrm{ted}_{0.5}$ at $298 \mathrm{~K}$. 


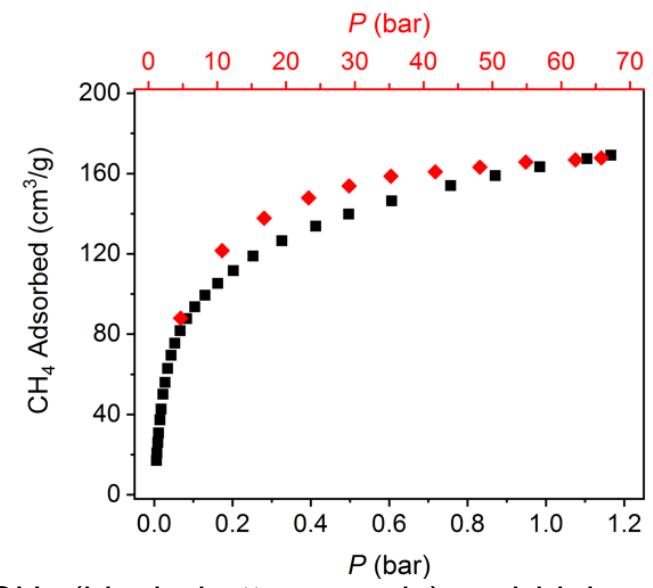

Figure S81. Low-pressure $\mathrm{CH}_{4}$ (black, bottom x-axis) and high-pressure $\mathrm{CH}_{4}$ (red, top x-axis) adsorption isotherms at $195 \mathrm{~K}$ and $298 \mathrm{~K}$ respectively, for $\mathrm{Ni}_{2}$ (m-dobdc).

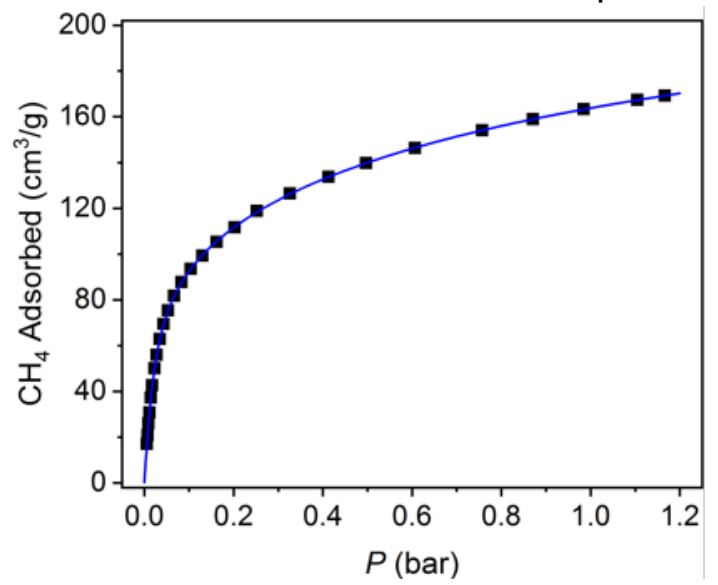

\begin{tabular}{|c|c|}
\hline $\mathrm{q}_{\mathrm{sat}, 1}$ & 52.048 \\
$\mathrm{~b}_{1}$ & 400.918 \\
\hline $\mathrm{v}_{1}$ & 1.525 \\
\hline $\mathrm{q}_{\mathrm{sat}, 2}$ & 240.088 \\
\hline $\mathrm{b}_{2}$ & 0.872 \\
\hline $\mathrm{v}_{2}$ & 0.585 \\
\hline $\mathrm{q}_{\mathrm{sat}, 3}$ & 0 \\
\hline $\mathrm{b}_{3}$ & 0 \\
\hline $\mathrm{v}_{3}$ & 0 \\
\hline
\end{tabular}

Figure S82. Low pressure $\mathrm{CH}_{4}$ isotherm with fit (left) and corresponding fitting parameters (right) for $\mathrm{Ni}_{2}(m$-dobdc) at $195 \mathrm{~K}$.

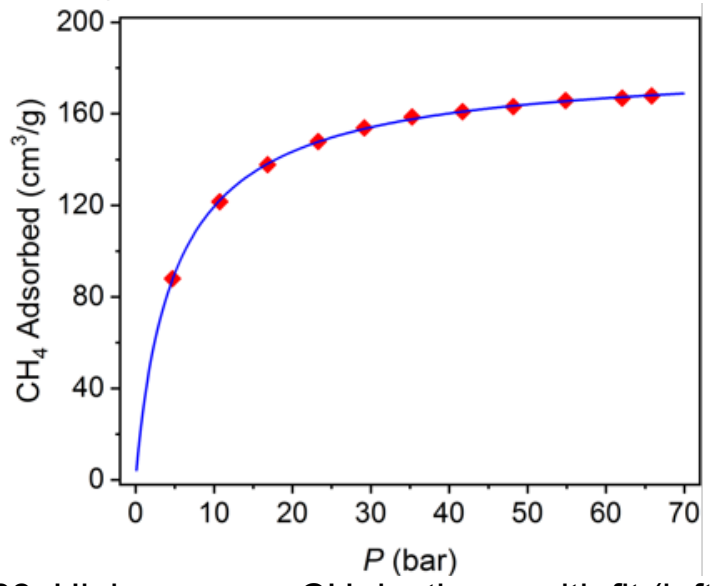

\begin{tabular}{|c|c|}
\hline $\mathrm{q}_{\mathrm{sat}, 1}$ & 183.165 \\
\hline $\mathrm{b}_{1}$ & 0.213 \\
\hline $\mathrm{v}_{1}$ & 0.945 \\
\hline $\mathrm{q}_{\mathrm{sat}, 2}$ & 0 \\
\hline $\mathrm{b}_{2}$ & 0 \\
\hline $\mathrm{v}_{2}$ & 0 \\
\hline $\mathrm{q}_{\mathrm{sat}, 3}$ & 0 \\
\hline $\mathrm{b}_{3}$ & 0 \\
\hline $\mathrm{v}_{3}$ & 0 \\
\hline
\end{tabular}

Figure S83. High pressure $\mathrm{CH}_{4}$ isotherm with fit (left) and corresponding fitting parameters (right) for $\mathrm{Ni}_{2}(m$-dobdc) at $298 \mathrm{~K}$. 


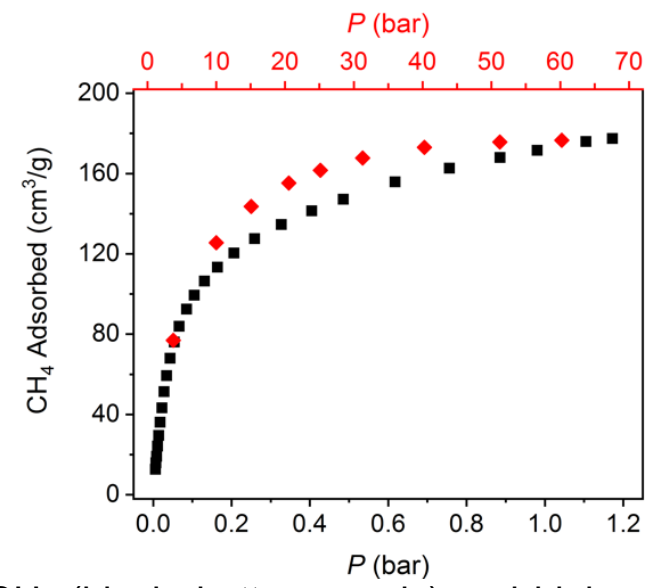

Figure S84. Low-pressure $\mathrm{CH}_{4}$ (black, bottom x-axis) and high-pressure $\mathrm{CH}_{4}$ (red, top x-axis) adsorption isotherms at $195 \mathrm{~K}$ and $298 \mathrm{~K}$ respectively, for Ni-MOF-74.

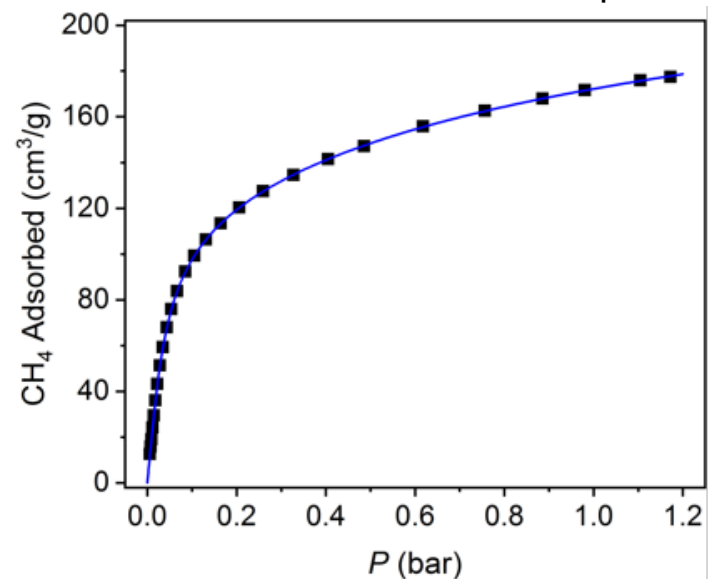

\begin{tabular}{|c|c|}
\hline$q_{\text {sat }, 1}$ & 219.036 \\
$\mathrm{~b}_{1}$ & 0.809 \\
\hline $\mathrm{v}_{1}$ & 0.636 \\
\hline $\mathrm{q}_{\mathrm{sat}, 2}$ & 74.673 \\
\hline $\mathrm{b}_{2}$ & 159.184 \\
\hline $\mathrm{v}_{2}$ & 1.465 \\
\hline $\mathrm{q}_{\mathrm{sat}, 3}$ & 0 \\
\hline $\mathrm{b}_{3}$ & 0 \\
\hline $\mathrm{v}_{3}$ & 0 \\
\hline
\end{tabular}

Figure S85. Low pressure $\mathrm{CH}_{4}$ isotherm with fit (left) and corresponding fitting parameters (right) for Ni-MOF-74 at $195 \mathrm{~K}$.

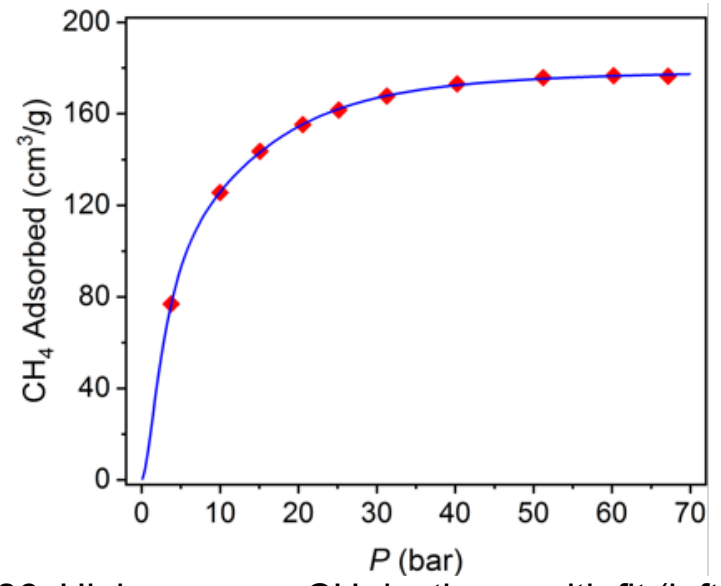

\begin{tabular}{|c|c|}
\hline $\mathrm{q}_{\mathrm{sat}, 1}$ & 40.732 \\
\hline $\mathrm{b}_{1}$ & $5.40 \mathrm{e}-4$ \\
\hline $\mathrm{v}_{1}$ & 2.594 \\
\hline $\mathrm{q}_{\mathrm{sat}, 2}$ & 138.811 \\
\hline $\mathrm{b}_{2}$ & 0.143 \\
\hline $\mathrm{v}_{2}$ & 1.613 \\
\hline $\mathrm{q}_{\mathrm{sat}, 3}$ & 0 \\
\hline $\mathrm{b}_{3}$ & 0 \\
\hline $\mathrm{v}_{3}$ & 0 \\
\hline
\end{tabular}

Figure S86. High pressure $\mathrm{CH}_{4}$ isotherm with fit (left) and corresponding fitting parameters (right) for Ni-MOF-74 at $298 \mathrm{~K}$. 


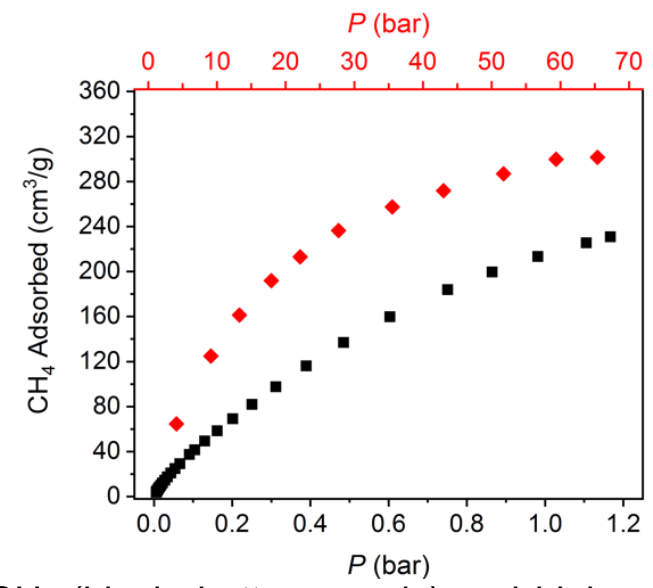

Figure S87. Low-pressure $\mathrm{CH}_{4}$ (black, bottom x-axis) and high-pressure $\mathrm{CH}_{4}$ (red, top x-axis) adsorption isotherms at $195 \mathrm{~K}$ and $298 \mathrm{~K}$ respectively, for NOTT-101.

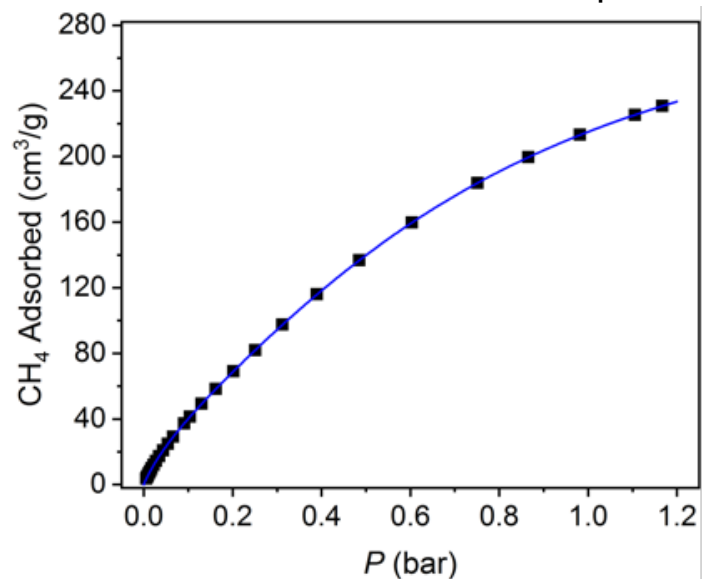

\begin{tabular}{|c|c|}
\hline $\mathrm{q}_{\mathrm{sat}, 1}$ & 77.381 \\
$\mathrm{~b}_{1}$ & 6.091 \\
\hline $\mathrm{v}_{1}$ & 0.944 \\
\hline $\mathrm{q}_{\mathrm{sat}, 2}$ & 246.590 \\
\hline $\mathrm{b}_{2}$ & 1.515 \\
\hline $\mathrm{v}_{2}$ & 1.632 \\
\hline $\mathrm{q}_{\mathrm{sat}, 3}$ & 0 \\
\hline $\mathrm{b}_{3}$ & 0 \\
\hline $\mathrm{v}_{3}$ & 0 \\
\hline
\end{tabular}

Figure S88. Low pressure $\mathrm{CH}_{4}$ isotherm with fit (left) and corresponding fitting parameters (right) for NOTT-101 at $195 \mathrm{~K}$.

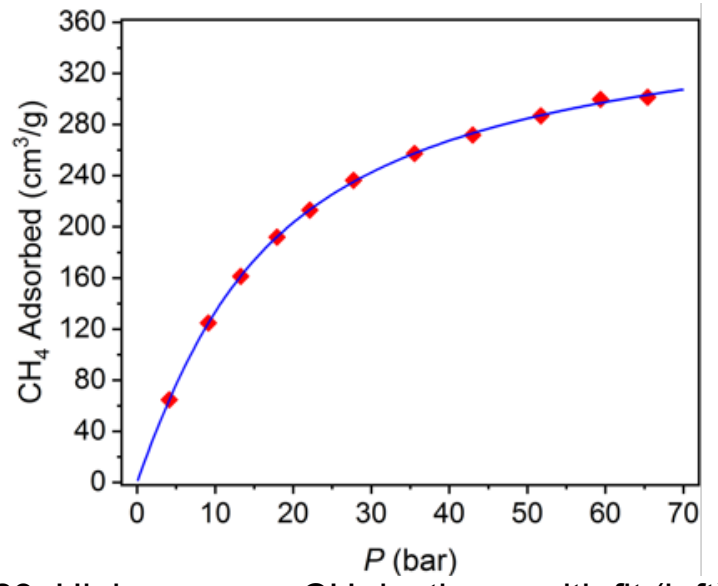

\begin{tabular}{|c|c|}
\hline $\mathrm{q}_{\mathrm{sat}, 1}$ & 82.656 \\
$\mathrm{~b}_{1}$ & 0.017 \\
\hline $\mathrm{v}_{1}$ & 1.613 \\
\hline $\mathrm{q}_{\mathrm{sat}, 2}$ & 305.556 \\
\hline $\mathrm{b}_{2}$ & 0.055 \\
\hline $\mathrm{v}_{2}$ & 0.944 \\
\hline $\mathrm{q}_{\mathrm{sat}, 3}$ & 0 \\
\hline $\mathrm{b}_{3}$ & 0 \\
\hline $\mathrm{v}_{3}$ & 0 \\
\hline
\end{tabular}

Figure S89. High pressure $\mathrm{CH}_{4}$ isotherm with fit (left) and corresponding fitting parameters (right) for NOTT-101 at $298 \mathrm{~K}$. 


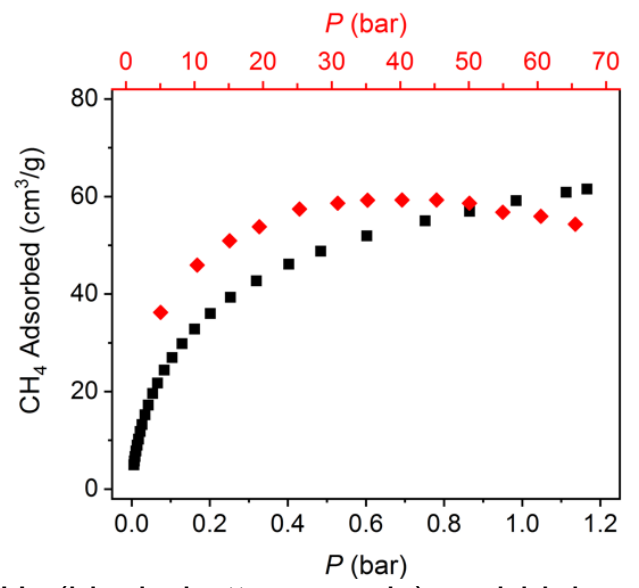

Figure S90. Low-pressure $\mathrm{CH}_{4}$ (black, bottom x-axis) and high-pressure $\mathrm{CH}_{4}$ (red, top x-axis) adsorption isotherms at $195 \mathrm{~K}$ and $298 \mathrm{~K}$ respectively, for (sc4a)Fe(bdc).

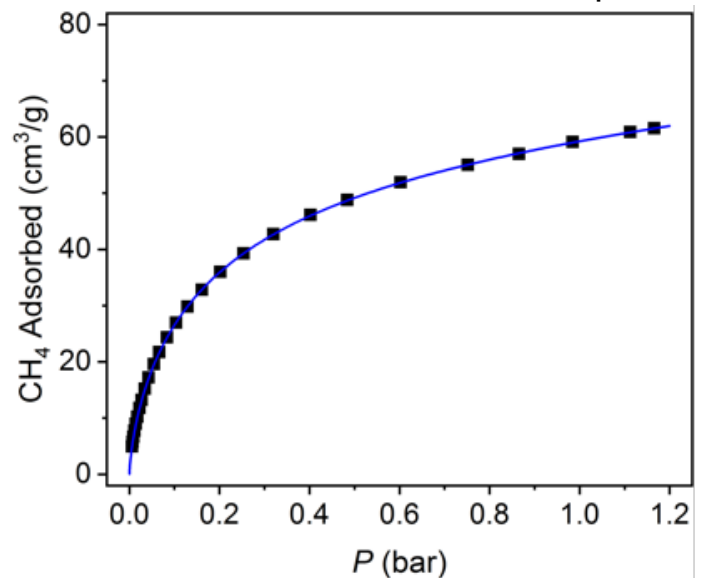

\begin{tabular}{|c|c|}
\hline $\mathrm{q}_{\mathrm{sat}, 1}$ & 78.769 \\
$\mathrm{~b}_{1}$ & 2.602 \\
\hline $\mathrm{v}_{1}$ & 0.711 \\
\hline $\mathrm{q}_{\mathrm{sat}, 2}$ & 499.998 \\
\hline $\mathrm{b}_{2}$ & 0.005 \\
\hline $\mathrm{v}_{2}$ & 1.527 \\
\hline $\mathrm{q}_{\mathrm{sat}, 3}$ & 0 \\
\hline $\mathrm{b}_{3}$ & 0 \\
\hline $\mathrm{v}_{3}$ & 0 \\
\hline
\end{tabular}

Figure S91. Low pressure $\mathrm{CH}_{4}$ isotherm with fit (left) and corresponding fitting parameters (right) for (sc4a)Fe(bdc) at $195 \mathrm{~K}$.

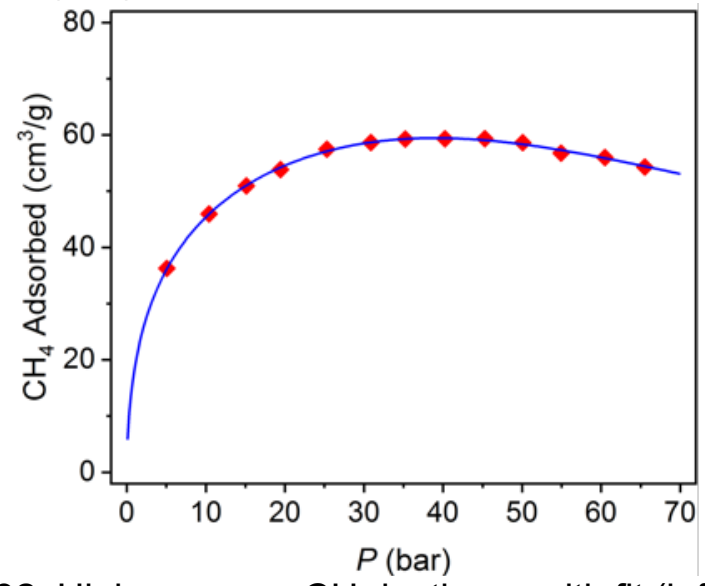

\begin{tabular}{|c|c|}
\hline $\mathrm{q}_{\mathrm{sat}, 1}$ & 219.450 \\
\hline $\mathrm{b}_{1}$ & 0.089 \\
\hline $\mathrm{v}_{1}$ & 0.870 \\
\hline $\mathrm{q}_{\mathrm{sat}, 2}$ & 0 \\
\hline $\mathrm{b}_{2}$ & 0 \\
\hline $\mathrm{v}_{2}$ & 0 \\
\hline $\mathrm{q}_{\mathrm{sat}, 3}$ & 0 \\
\hline $\mathrm{b}_{3}$ & 0 \\
\hline $\mathrm{v}_{3}$ & 0 \\
\hline
\end{tabular}

Figure S92. High pressure $\mathrm{CH}_{4}$ isotherm with fit (left) and corresponding fitting parameters (right) for (sc4a)Fe(bdc) at $298 \mathrm{~K}$. 


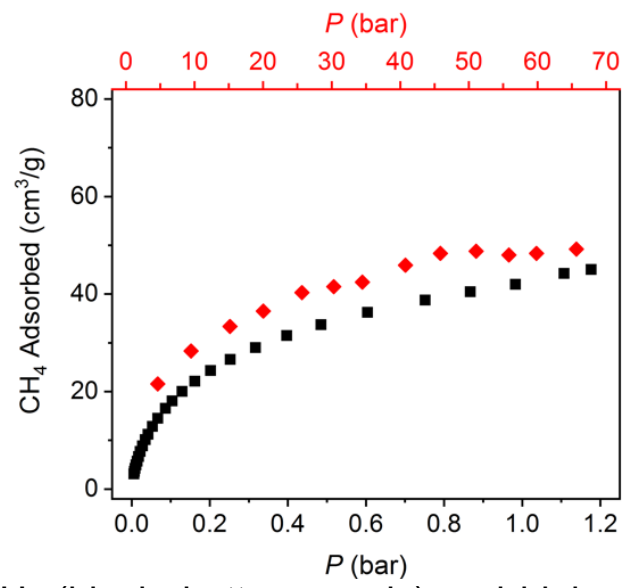

Figure S93. Low-pressure $\mathrm{CH}_{4}$ (black, bottom x-axis) and high-pressure $\mathrm{CH}_{4}$ (red, top x-axis) adsorption isotherms at $195 \mathrm{~K}$ and $298 \mathrm{~K}$ respectively, for (sc4a) Fe(btc).

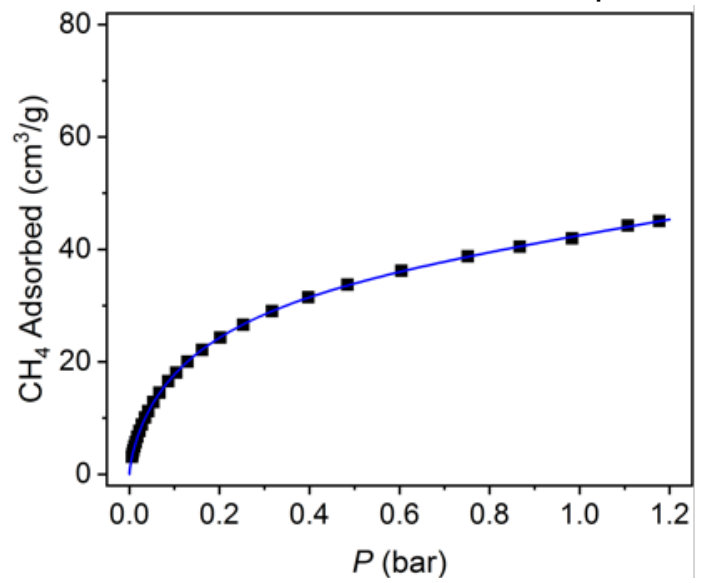

\begin{tabular}{|c|c|}
\hline $\mathrm{q}_{\mathrm{sat}, 1}$ & 53.435 \\
\hline $\mathrm{b}_{1}$ & 2.656 \\
\hline $\mathrm{v}_{1}$ & 0.730 \\
\hline $\mathrm{q}_{\mathrm{sat}, 2}$ & 500 \\
\hline $\mathrm{b}_{2}$ & 0.0074 \\
\hline $\mathrm{v}_{2}$ & 1.851 \\
\hline $\mathrm{q}_{\mathrm{sat}, 3}$ & 0 \\
\hline $\mathrm{b}_{3}$ & 0 \\
\hline $\mathrm{v}_{3}$ & 0 \\
\hline
\end{tabular}

Figure S94. Low pressure $\mathrm{CH}_{4}$ isotherm with fit (left) and corresponding fitting parameters (right) for $(\mathrm{sc} 4 \mathrm{a}) \mathrm{Fe}(\mathrm{btc})$ at $195 \mathrm{~K}$.

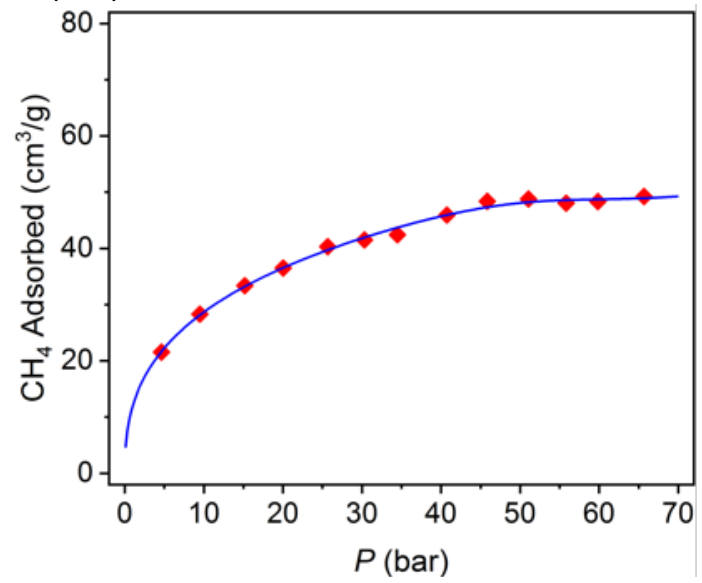

\begin{tabular}{|c|c|}
\hline $\mathrm{q}_{\mathrm{sat}, 1}$ & 191.566 \\
\hline $\mathrm{b}_{1}$ & 0.067 \\
\hline $\mathrm{v}_{1}$ & 0.420 \\
\hline $\mathrm{q}_{\mathrm{sat}, 2}$ & -6.788 \\
\hline $\mathrm{b}_{2}$ & $6.50 \mathrm{e}-16$ \\
\hline $\mathrm{v}_{2}$ & 8.540 \\
\hline $\mathrm{q}_{\mathrm{sat}, 3}$ & 0 \\
\hline $\mathrm{b}_{3}$ & 0 \\
\hline $\mathrm{v}_{3}$ & 0 \\
\hline
\end{tabular}

Figure S95. High pressure $\mathrm{CH}_{4}$ isotherm with fit (left) and corresponding fitting parameters (right) for (sc4a)Fe(btc) at $298 \mathrm{~K}$. 


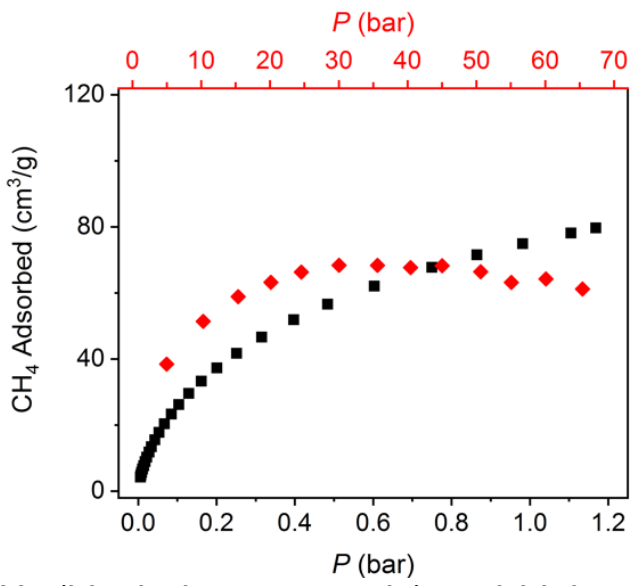

Figure S96. Low-pressure $\mathrm{CH}_{4}$ (black, bottom x-axis) and high-pressure $\mathrm{CH}_{4}$ (red, top x-axis) adsorption isotherms at $195 \mathrm{~K}$ and $298 \mathrm{~K}$ respectively, for (sc4a)Fe(tatb).

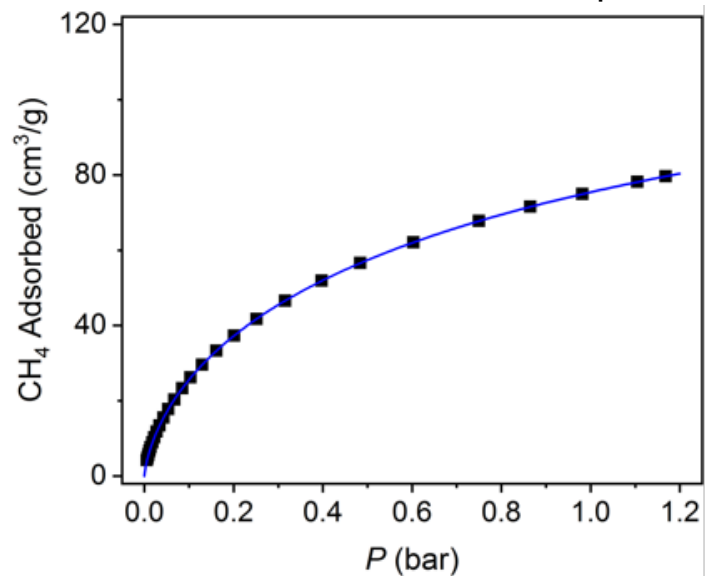

\begin{tabular}{|c|c|}
\hline $\mathrm{q}_{\mathrm{sat}, 1}$ & 12.709 \\
$\mathrm{~b}_{1}$ & 5.130 \\
\hline $\mathrm{v}_{1}$ & 0.751 \\
\hline $\mathrm{q}_{\mathrm{sat}, 2}$ & 145.248 \\
\hline $\mathrm{b}_{2}$ & 0.805 \\
\hline $\mathrm{v}_{2}$ & 0.714 \\
\hline $\mathrm{q}_{\mathrm{sat}, 3}$ & 0 \\
\hline $\mathrm{b}_{3}$ & 0 \\
\hline $\mathrm{v}_{3}$ & 0 \\
\hline
\end{tabular}

Figure S97. Low pressure $\mathrm{CH}_{4}$ isotherm with fit (left) and corresponding fitting parameters (right) for (sc4a)Fe(tatb) at $195 \mathrm{~K}$.

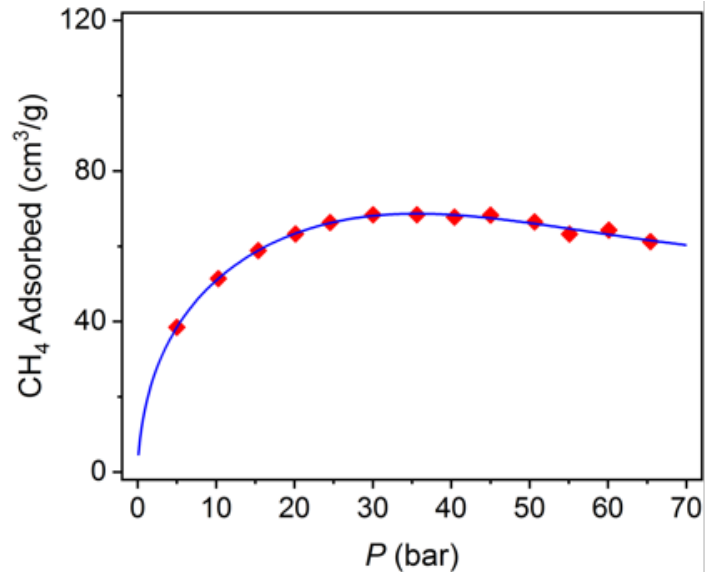

\begin{tabular}{|c|c|}
\hline $\mathrm{q}_{\mathrm{sat}, 1}$ & 132.773 \\
\hline $\mathrm{b}_{1}$ & 0.152 \\
\hline $\mathrm{v}_{1}$ & 0.616 \\
\hline $\mathrm{q}_{\mathrm{sat}, 2}$ & -50 \\
\hline $\mathrm{b}_{2}$ & $5.00 \mathrm{e}-6$ \\
\hline $\mathrm{v}_{2}$ & 2.954 \\
\hline $\mathrm{q}_{\mathrm{sat}, 3}$ & 0 \\
\hline $\mathrm{b}_{3}$ & 0 \\
\hline $\mathrm{v}_{3}$ & 0 \\
\hline
\end{tabular}

Figure S98. High pressure $\mathrm{CH}_{4}$ isotherm with fit (left) and corresponding fitting parameters (right) for (sc4a)Fe(tatb) at $298 \mathrm{~K}$. 


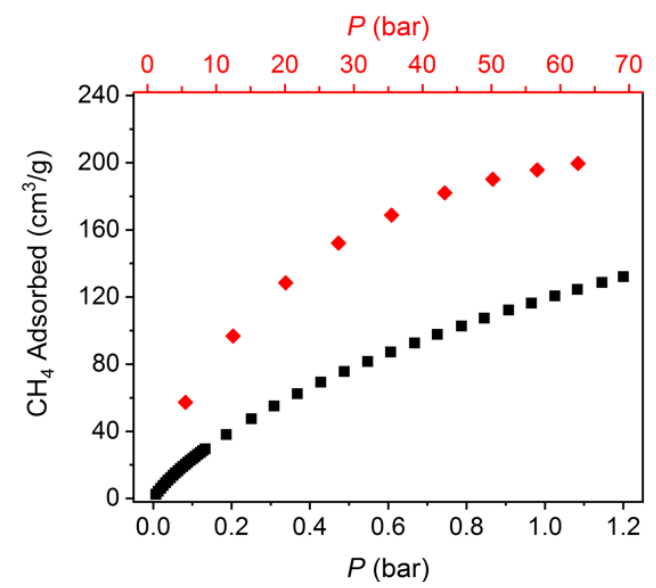

Figure S99. Low-pressure $\mathrm{CH}_{4}$ (black, bottom x-axis) and high-pressure $\mathrm{CH}_{4}$ (red, top x-axis) adsorption isotherms at $195 \mathrm{~K}$ and $298 \mathrm{~K}$ respectively, for Ti-MIL-101.

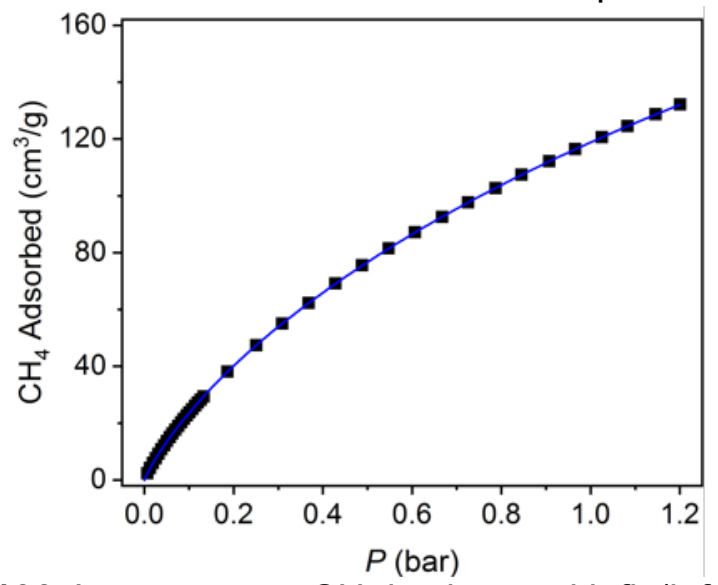

\begin{tabular}{|c|c|}
\hline $\mathrm{q}_{\mathrm{sat}, 1}$ & 371.168 \\
$\mathrm{~b}_{1}$ & 0.364 \\
\hline $\mathrm{v}_{1}$ & 0.913 \\
\hline $\mathrm{q}_{\mathrm{sat}, 2}$ & 25.360 \\
\hline $\mathrm{b}_{2}$ & 3.552 \\
\hline $\mathrm{v}_{2}$ & 0.906 \\
\hline $\mathrm{q}_{\mathrm{sat}, 3}$ & 0 \\
\hline $\mathrm{b}_{3}$ & 0 \\
\hline $\mathrm{v}_{3}$ & 0 \\
\hline
\end{tabular}

Figure S100. Low pressure $\mathrm{CH}_{4}$ isotherm with fit (left) and corresponding fitting parameters (right) for Ti-MIL-101 at $195 \mathrm{~K}$.

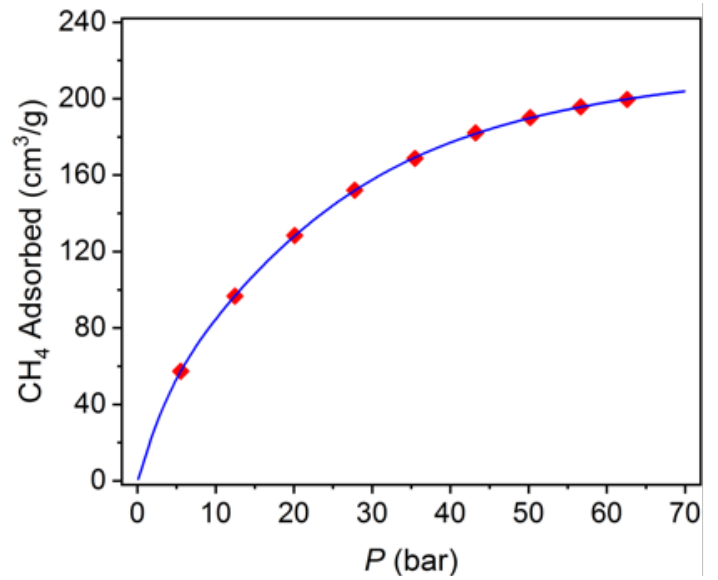

\begin{tabular}{|c|c|}
\hline $\mathrm{q}_{\mathrm{sat}, 1}$ & 115.048 \\
$\mathrm{~b}_{1}$ & 0.122 \\
\hline $\mathrm{v}_{1}$ & 1.145 \\
\hline $\mathrm{q}_{\mathrm{sat}, 2}$ & 110.822 \\
\hline $\mathrm{b}_{2}$ & 0.001 \\
\hline $\mathrm{v}_{2}$ & 2.025 \\
\hline $\mathrm{q}_{\mathrm{sat}, 3}$ & 0 \\
\hline $\mathrm{b}_{3}$ & 0 \\
\hline $\mathrm{v}_{3}$ & 0 \\
\hline
\end{tabular}

Figure S101. High pressure $\mathrm{CH}_{4}$ isotherm with fit (left) and corresponding fitting parameters (right) for Ti-MIL-101 at $298 \mathrm{~K}$. 


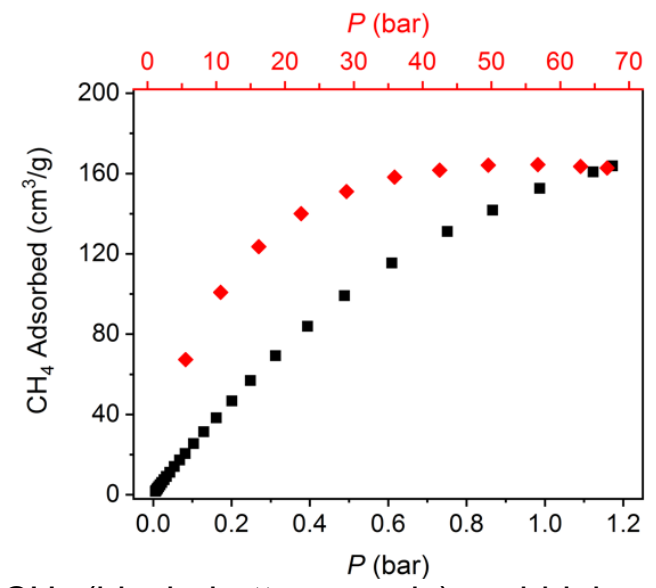

Figure S102. Low-pressure $\mathrm{CH}_{4}$ (black, bottom x-axis) and high-pressure $\mathrm{CH}_{4}$ (red, top x-axis) adsorption isotherms at $195 \mathrm{~K}$ and $298 \mathrm{~K}$ respectively, for Ti-MIL-125.

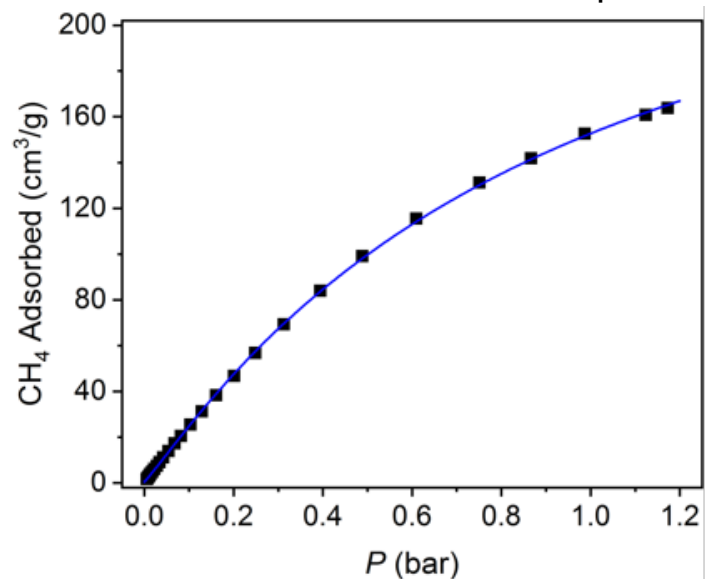

\begin{tabular}{|c|c|}
\hline $\mathrm{q}_{\mathrm{sat}, 1}$ & 0.537 \\
$\mathrm{~b}_{1}$ & $4.62 \mathrm{e} 22$ \\
\hline $\mathrm{v}_{1}$ & 4.359 \\
\hline $\mathrm{q}_{\mathrm{sat}, 2}$ & 292.608 \\
\hline $\mathrm{b}_{2}$ & 1.083 \\
\hline $\mathrm{v}_{2}$ & 1.078 \\
\hline $\mathrm{q}_{\mathrm{sat}, 3}$ & 0 \\
\hline $\mathrm{b}_{3}$ & 0 \\
\hline $\mathrm{v}_{3}$ & 0 \\
\hline
\end{tabular}

Figure S103. Low pressure $\mathrm{CH}_{4}$ isotherm with fit (left) and corresponding fitting parameters (right) for Ti-MIL-125 at $195 \mathrm{~K}$.

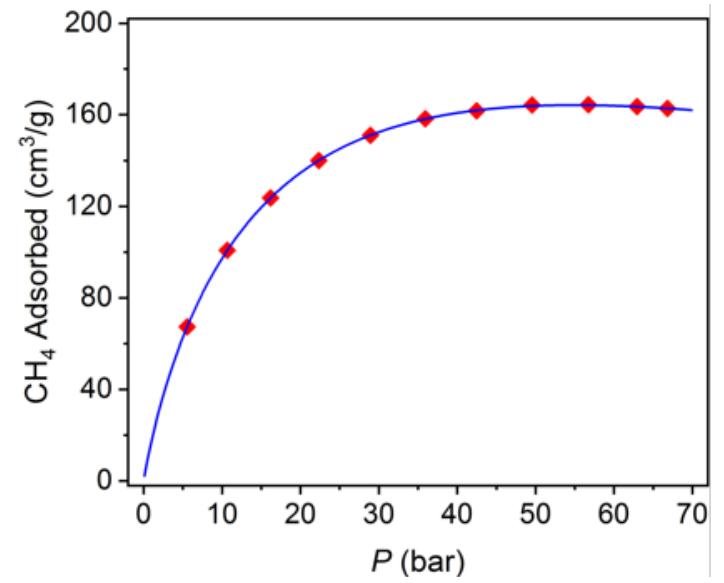

\begin{tabular}{|c|c|}
\hline $\mathrm{q}_{\mathrm{sat}, 1}$ & 333.564 \\
\hline $\mathrm{b}_{1}$ & 0.059 \\
\hline $\mathrm{v}_{1}$ & 0.920 \\
\hline $\mathrm{q}_{\mathrm{sat}, 2}$ & -500 \\
\hline $\mathrm{b}_{2}$ & 0.002 \\
\hline $\mathrm{v}_{2}$ & 1.060 \\
\hline $\mathrm{q}_{\mathrm{sat}, 3}$ & 0 \\
\hline $\mathrm{b}_{3}$ & 0 \\
\hline $\mathrm{v}_{3}$ & 0 \\
\hline
\end{tabular}

Figure S104. High pressure $\mathrm{CH}_{4}$ isotherm with fit (left) and corresponding fitting parameters (right) for Ti-MIL-125 at $298 \mathrm{~K}$. 


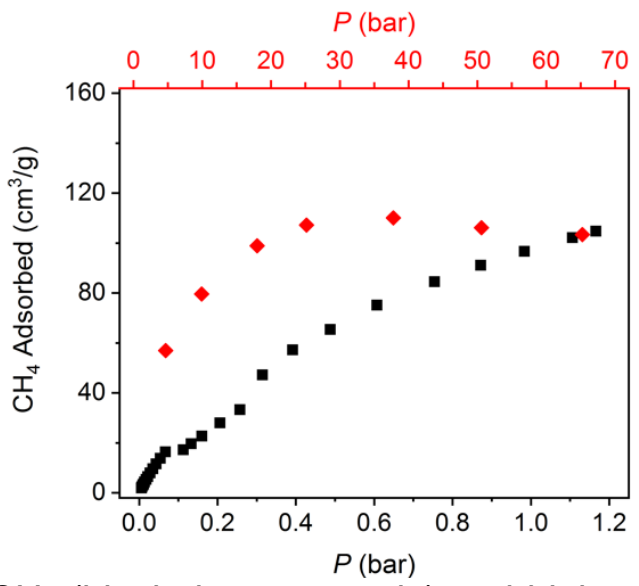

Figure S105. Low-pressure $\mathrm{CH}_{4}$ (black, bottom x-axis) and high-pressure $\mathrm{CH}_{4}$ (red, top x-axis) adsorption isotherms at $195 \mathrm{~K}$ and $298 \mathrm{~K}$ respectively, for UiO-66 defect free.

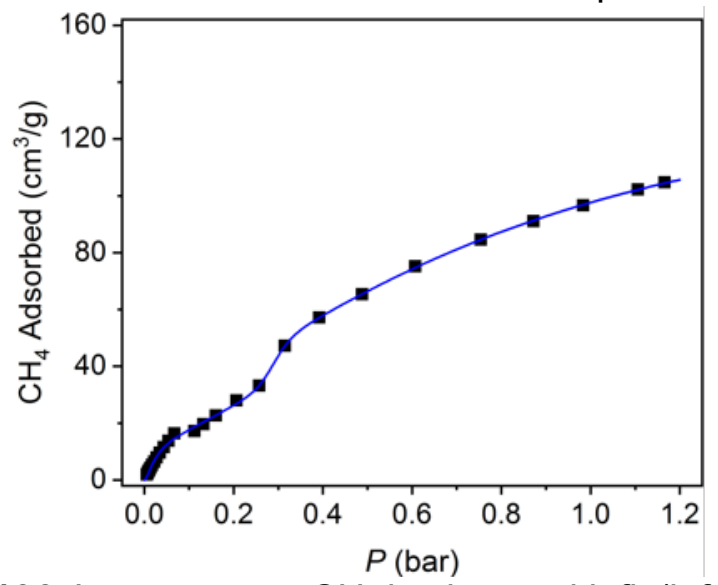

\begin{tabular}{|c|c|}
\hline $\mathrm{q}_{\mathrm{sat}, 1}$ & 15.413 \\
$\mathrm{~b}_{1}$ & 243.711 \\
\hline $\mathrm{v}_{1}$ & 1.525 \\
\hline $\mathrm{q}_{\mathrm{sat}, 2}$ & 117.312 \\
\hline $\mathrm{b}_{2}$ & 1.393 \\
\hline $\mathrm{v}_{2}$ & 1.584 \\
\hline $\mathrm{q}_{\mathrm{sat}, 3}$ & 14.006 \\
\hline $\mathrm{b}_{3}$ & $8.41 \mathrm{e} 7$ \\
\hline $\mathrm{v}_{3}$ & 14.781 \\
\hline
\end{tabular}

Figure S106. Low pressure $\mathrm{CH}_{4}$ isotherm with fit (left) and corresponding fitting parameters (right) for UiO-66 defect free at $195 \mathrm{~K}$.

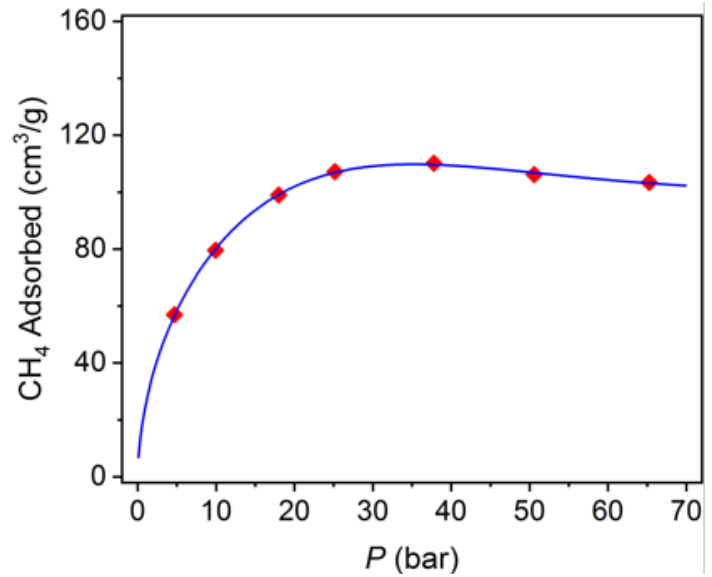

\begin{tabular}{|c|c|}
\hline $\mathrm{q}_{\mathrm{sat}, 1}$ & 500 \\
$\mathrm{~b}_{1}$ & 0.053 \\
\hline $\mathrm{v}_{1}$ & 0.578 \\
\hline $\mathrm{q}_{\mathrm{sat}, 2}$ & -150.713 \\
\hline $\mathrm{b}_{2}$ & $1.49 \mathrm{e}-4$ \\
\hline $\mathrm{v}_{2}$ & 2.157 \\
\hline $\mathrm{q}_{\mathrm{sat}, 3}$ & 0 \\
\hline $\mathrm{b}_{3}$ & 0 \\
\hline $\mathrm{v}_{3}$ & 0 \\
\hline
\end{tabular}

Figure S107. High pressure $\mathrm{CH}_{4}$ isotherm with fit (left) and corresponding fitting parameters (right) for UiO-66 defect free at $298 \mathrm{~K}$. 


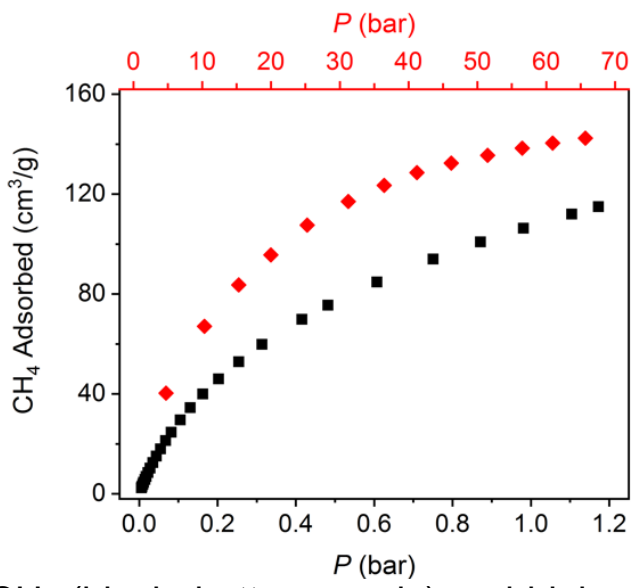

Figure S108. Low-pressure $\mathrm{CH}_{4}$ (black, bottom x-axis) and high-pressure $\mathrm{CH}_{4}$ (red, top x-axis) adsorption isotherms at $195 \mathrm{~K}$ and $298 \mathrm{~K}$ respectively, for UiO-66 high surface area.

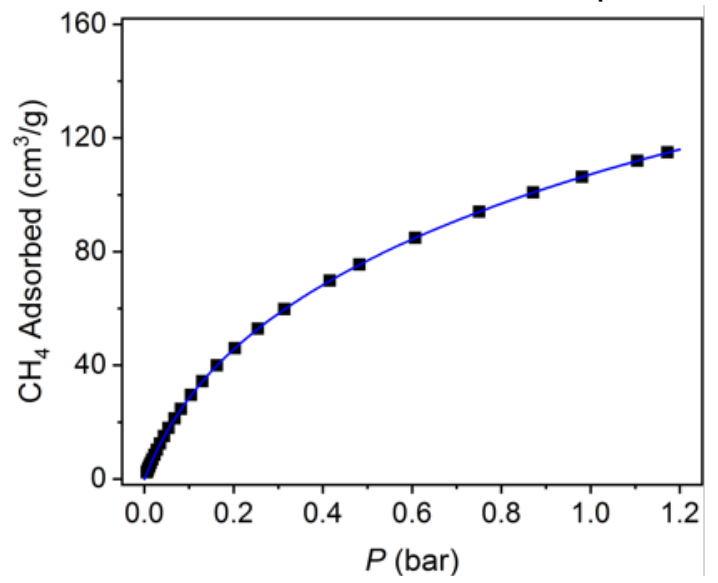

\begin{tabular}{|c|c|}
\hline $\mathrm{q}_{\mathrm{sat}, 1}$ & 49.268 \\
$\mathrm{~b}_{1}$ & 5.798 \\
\hline $\mathrm{v}_{1}$ & 0.996 \\
\hline $\mathrm{q}_{\mathrm{sat}, 2}$ & 191.057 \\
\hline $\mathrm{b}_{2}$ & 0.518 \\
\hline $\mathrm{v}_{2}$ & 0.957 \\
\hline $\mathrm{q}_{\mathrm{sat}, 3}$ & 0 \\
\hline $\mathrm{b}_{3}$ & 0 \\
\hline $\mathrm{v}_{3}$ & 0 \\
\hline
\end{tabular}

Figure S109. Low pressure $\mathrm{CH}_{4}$ isotherm with fit (left) and corresponding fitting parameters (right) for UiO-66 high surface area at $195 \mathrm{~K}$.

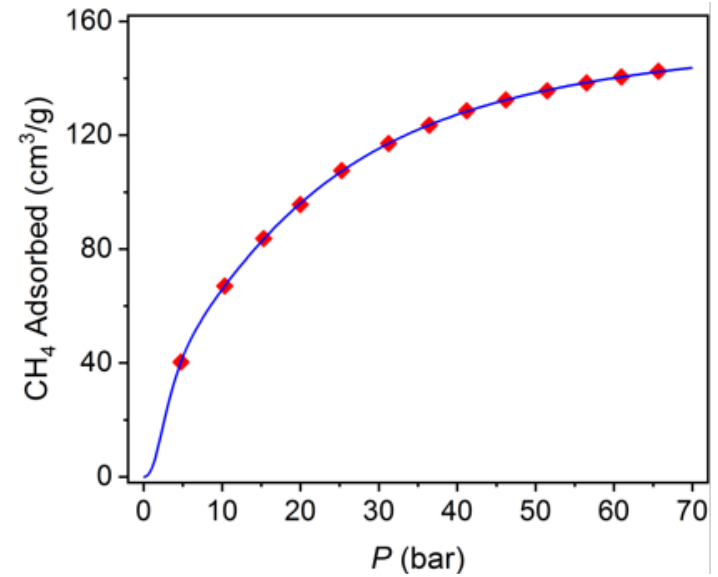

\begin{tabular}{|c|c|}
\hline $\mathrm{q}_{\mathrm{sat}, 1}$ & 114.515 \\
$\mathrm{~b}_{1}$ & 0.006 \\
\hline $\mathrm{v}_{1}$ & 1.659 \\
\hline $\mathrm{q}_{\mathrm{sat}, 2}$ & 43.837 \\
\hline $\mathrm{b}_{2}$ & 0.053 \\
\hline $\mathrm{v}_{2}$ & 2.545 \\
\hline $\mathrm{q}_{\mathrm{sat}, 3}$ & 0 \\
\hline $\mathrm{b}_{3}$ & 0 \\
\hline $\mathrm{v}_{3}$ & 0 \\
\hline
\end{tabular}

Figure S110. High pressure $\mathrm{CH}_{4}$ isotherm with fit (left) and corresponding fitting parameters (right) for UiO-66 high surface area at $298 \mathrm{~K}$. 


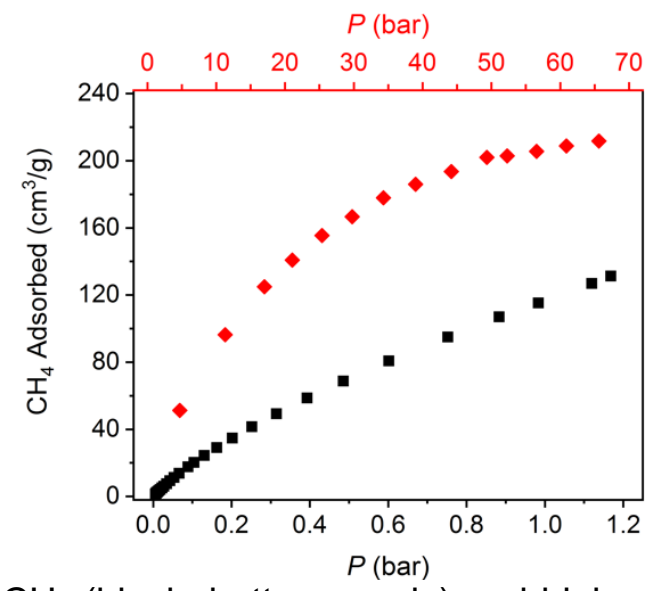

Figure S111. Low-pressure $\mathrm{CH}_{4}$ (black, bottom x-axis) and high-pressure $\mathrm{CH}_{4}$ (red, top x-axis) adsorption isotherms at $195 \mathrm{~K}$ and $298 \mathrm{~K}$ respectively, for UiO-67.

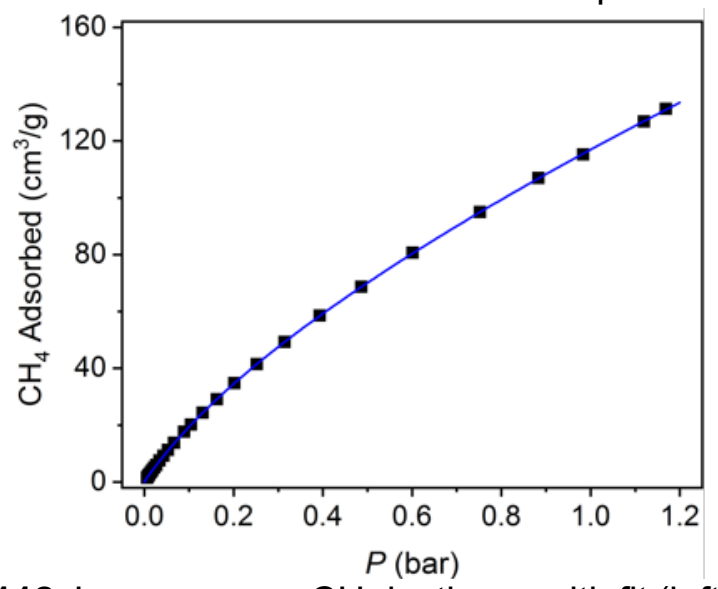

\begin{tabular}{|c|c|}
\hline $\mathrm{q}_{\mathrm{sat}, 1}$ & 80.150 \\
$\mathrm{~b}_{1}$ & 2.015 \\
\hline $\mathrm{v}_{1}$ & 0.934 \\
\hline $\mathrm{q}_{\mathrm{sat}, 2}$ & 500 \\
\hline $\mathrm{b}_{2}$ & 0.145 \\
\hline $\mathrm{v}_{2}$ & 1.238 \\
\hline $\mathrm{q}_{\mathrm{sat}, 3}$ & 0 \\
\hline $\mathrm{b}_{3}$ & 0 \\
\hline $\mathrm{v}_{3}$ & 0 \\
\hline
\end{tabular}

Figure S112. Low pressure $\mathrm{CH}_{4}$ isotherm with fit (left) and corresponding fitting parameters (right) for $\mathrm{UiO}-67$ at $195 \mathrm{~K}$.

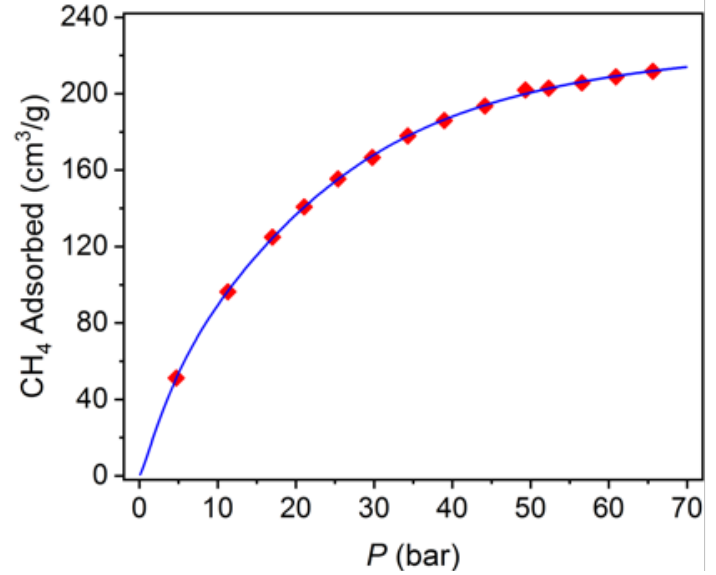

\begin{tabular}{|c|c|}
\hline $\mathrm{q}_{\mathrm{sat}, 1}$ & 90.205 \\
$\mathrm{~b}_{1}$ & $5.36 \mathrm{e}-4$ \\
\hline $\mathrm{v}_{1}$ & 2.263 \\
\hline $\mathrm{q}_{\mathrm{sat}, 2}$ & 143.614 \\
\hline $\mathrm{b}_{2}$ & 0.081 \\
\hline $\mathrm{v}_{2}$ & 1.205 \\
\hline $\mathrm{q}_{\mathrm{sat}, 3}$ & 0 \\
\hline $\mathrm{b}_{3}$ & 0 \\
\hline $\mathrm{v}_{3}$ & 0 \\
\hline
\end{tabular}

Figure S113. High pressure $\mathrm{CH}_{4}$ isotherm with fit (left) and corresponding fitting parameters (right) for UiO-67 at $298 \mathrm{~K}$. 


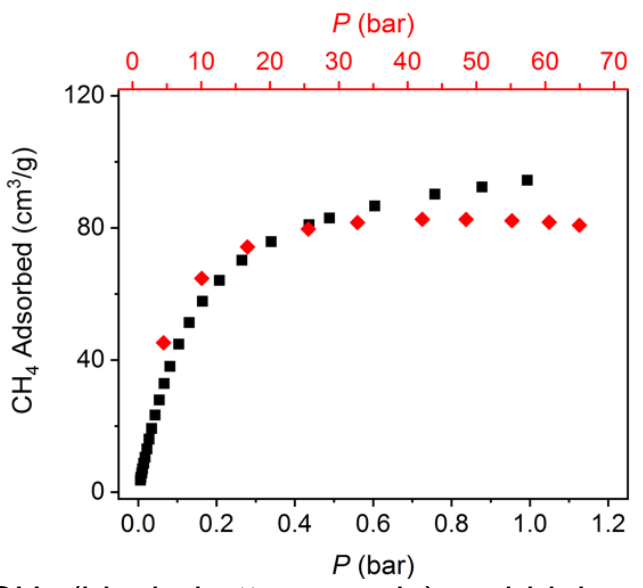

Figure S114. Low-pressure $\mathrm{CH}_{4}$ (black, bottom x-axis) and high-pressure $\mathrm{CH}_{4}$ (red, top x-axis) adsorption isotherms at $195 \mathrm{~K}$ and $298 \mathrm{~K}$ respectively, for Zeolite $5 \AA$.

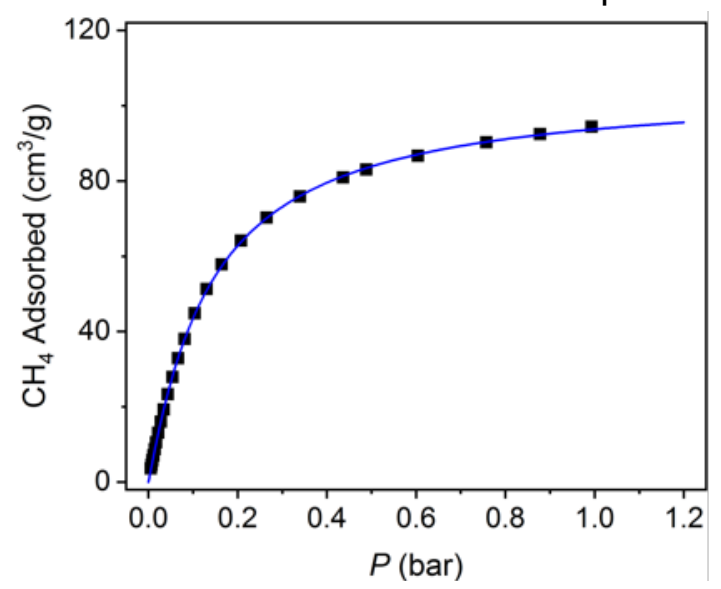

\begin{tabular}{|c|c|}
\hline $\mathrm{q}_{\mathrm{sat}, 1}$ & 104.955 \\
$\mathrm{~b}_{1}$ & 8.342 \\
\hline $\mathrm{v}_{1}$ & 1.071 \\
\hline $\mathrm{q}_{\mathrm{sat}, 2}$ & 0 \\
\hline $\mathrm{b}_{2}$ & 0 \\
\hline $\mathrm{v}_{2}$ & 0 \\
\hline $\mathrm{q}_{\mathrm{sat}, 3}$ & 0 \\
\hline $\mathrm{b}_{3}$ & 0 \\
\hline $\mathrm{v}_{3}$ & 0 \\
\hline
\end{tabular}

Figure S115. Low pressure $\mathrm{CH}_{4}$ isotherm with fit (left) and corresponding fitting parameters (right) for Zeolite $5 \AA$ at $195 \mathrm{~K}$.

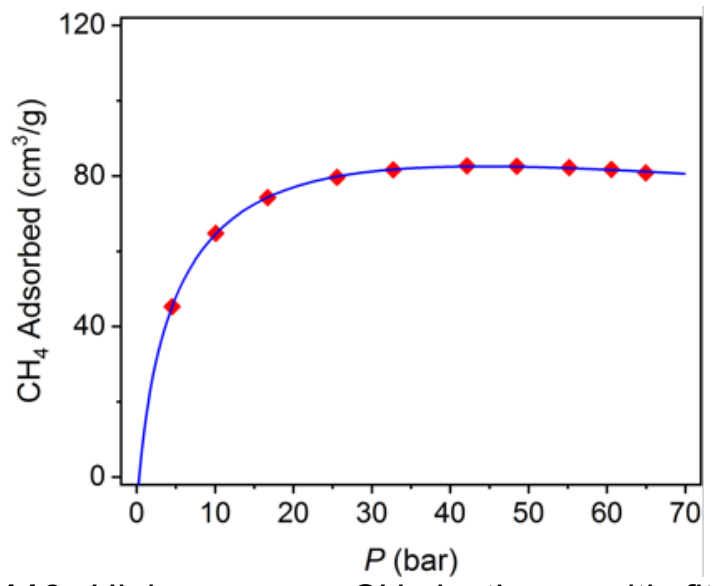

\begin{tabular}{|c|c|}
\hline $\mathrm{q}_{\mathrm{sat}, 1}$ & 258.241 \\
$\mathrm{~b}_{1}$ & 0.235 \\
\hline $\mathrm{v}_{1}$ & 0.702 \\
\hline $\mathrm{q}_{\mathrm{sat}, 2}$ & -500 \\
\hline $\mathrm{b}_{2}$ & 0.078 \\
\hline $\mathrm{v}_{2}$ & 0.359 \\
\hline $\mathrm{q}_{\mathrm{sat}, 3}$ & 0 \\
\hline $\mathrm{b}_{3}$ & 0 \\
\hline $\mathrm{v}_{3}$ & 0 \\
\hline
\end{tabular}

Figure S116. High pressure $\mathrm{CH}_{4}$ isotherm with fit (left) and corresponding fitting parameters (right) for Zeolite $5 \AA$ at $298 \mathrm{~K}$. 


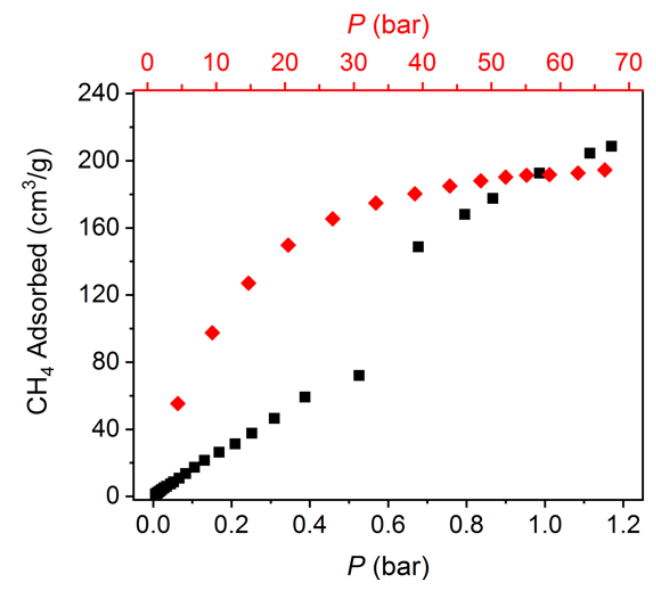

Figure S117. Low-pressure $\mathrm{CH}_{4}$ (black, bottom x-axis) and high-pressure $\mathrm{CH}_{4}$ (red, top x-axis) adsorption isotherms at $195 \mathrm{~K}$ and $298 \mathrm{~K}$ respectively, for $\mathrm{Zn}(\mathrm{bdc})(\mathrm{ted})_{0.5}$.

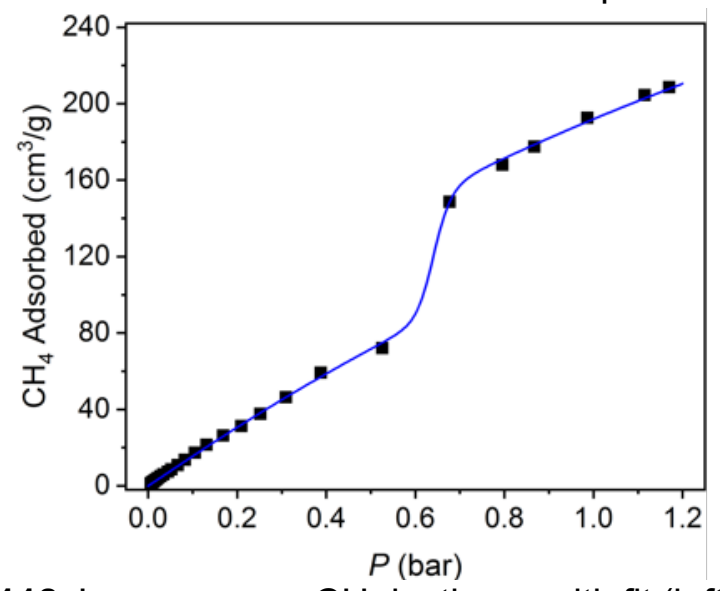

\begin{tabular}{|c|c|}
\hline $\mathrm{q}_{\mathrm{sat}, 1}$ & 64.939 \\
$\mathrm{~b}_{1}$ & $2.87 \mathrm{e} 6$ \\
\hline $\mathrm{v}_{1}$ & 33.530 \\
\hline $\mathrm{q}_{\mathrm{sat}, 2}$ & 500 \\
\hline $\mathrm{b}_{2}$ & 0.340 \\
\hline $\mathrm{v}_{2}$ & 1.026 \\
\hline $\mathrm{q}_{\mathrm{sat}, 3}$ & 0 \\
\hline $\mathrm{b}_{3}$ & 0 \\
\hline $\mathrm{v}_{3}$ & 0 \\
\hline
\end{tabular}

Figure S118. Low pressure $\mathrm{CH}_{4}$ isotherm with fit (left) and corresponding fitting parameters (right) for $\mathrm{Zn}(\mathrm{bdc})(\mathrm{ted})_{0.5}$ at $195 \mathrm{~K}$.

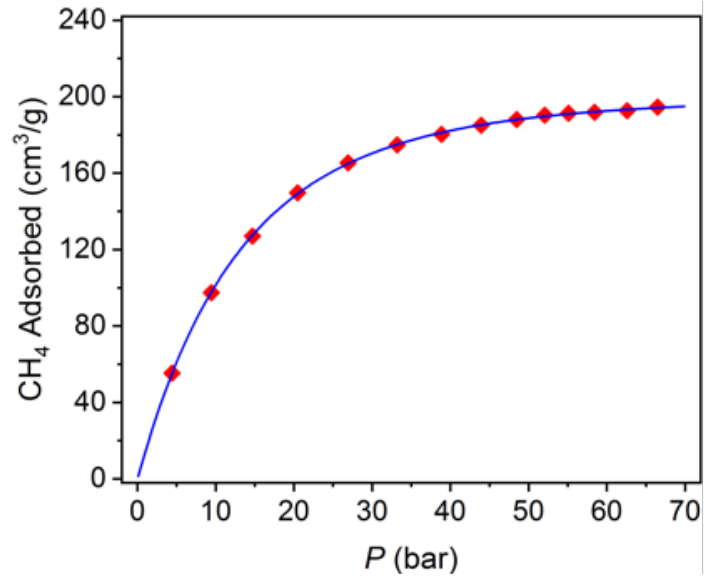

\begin{tabular}{|c|c|}
\hline $\mathrm{q}_{\mathrm{sat}, 1}$ & 500 \\
$\mathrm{~b}_{1}$ & 0.032 \\
\hline $\mathrm{v}_{1}$ & 1.038 \\
\hline $\mathrm{q}_{\mathrm{sat}, 2}$ & -286.17 \\
\hline $\mathrm{b}_{2}$ & 0.006 \\
\hline $\mathrm{v}_{2}$ & 1.303 \\
\hline $\mathrm{q}_{\mathrm{sat}, 3}$ & 0 \\
\hline $\mathrm{b}_{3}$ & 0 \\
\hline $\mathrm{v}_{3}$ & 0 \\
\hline
\end{tabular}

Figure S119. High pressure $\mathrm{CH}_{4}$ isotherm with fit (left) and corresponding fitting parameters (right) for $\mathrm{Zn}(\mathrm{bdc})(\mathrm{ted})_{0.5}$ at $298 \mathrm{~K}$. 


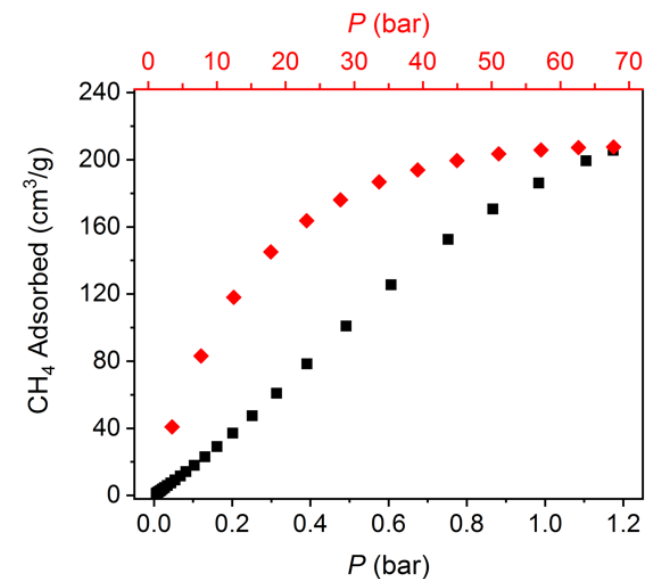

Figure S120. Low-pressure $\mathrm{CH}_{4}$ (black, bottom x-axis) and high-pressure $\mathrm{CH}_{4}$ (red, top x-axis) adsorption isotherms at $195 \mathrm{~K}$ and $298 \mathrm{~K}$ respectively, for $\mathrm{Zn}(\mathrm{bdc})_{2} \mathrm{dabco}$.

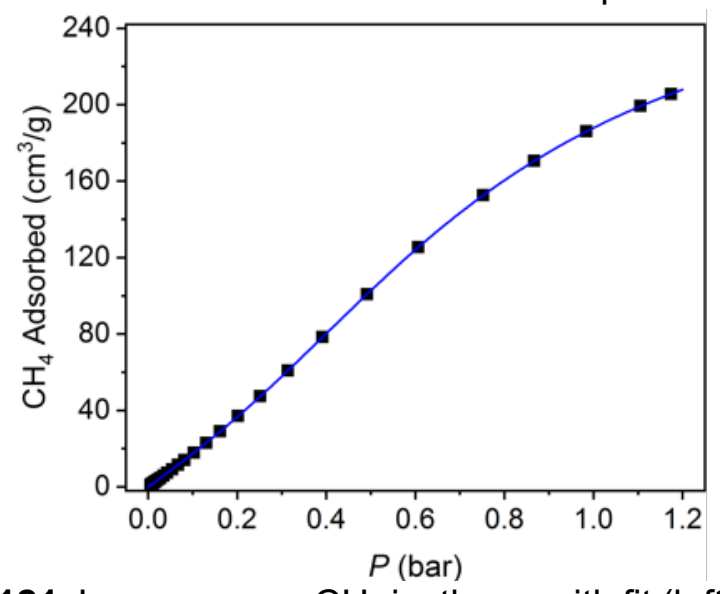

\begin{tabular}{|c|c|}
\hline $\mathrm{q}_{\mathrm{sat}, 1}$ & 169.542 \\
$\mathrm{~b}_{1}$ & 1.928 \\
\hline $\mathrm{v}_{1}$ & 2.257 \\
\hline $\mathrm{q}_{\mathrm{sat}, 2}$ & 124.464 \\
\hline $\mathrm{b}_{2}$ & 1.578 \\
\hline $\mathrm{v}_{2}$ & 1.042 \\
\hline $\mathrm{q}_{\mathrm{sat}, 3}$ & 0 \\
\hline $\mathrm{b}_{3}$ & 0 \\
\hline $\mathrm{v}_{3}$ & 0 \\
\hline
\end{tabular}

Figure S121. Low pressure $\mathrm{CH}_{4}$ isotherm with fit (left) and corresponding fitting parameters (right) for $\mathrm{Zn}(\mathrm{bdc})_{2} \mathrm{dabco}$ at $195 \mathrm{~K}$.

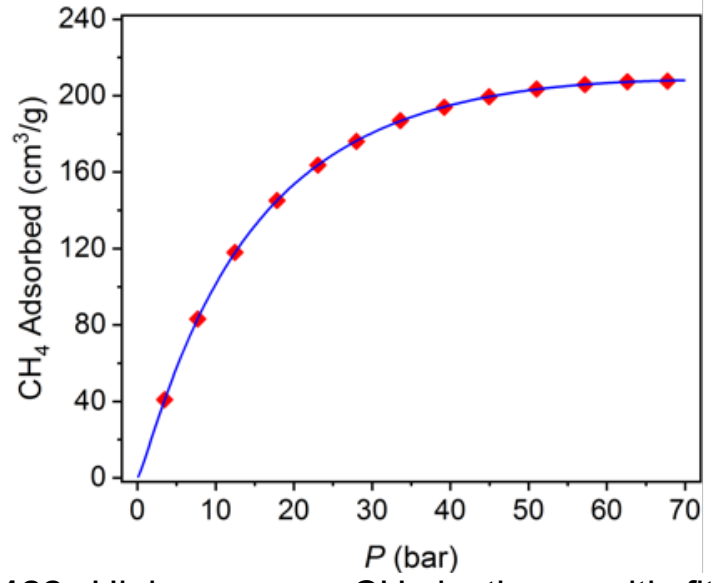

\begin{tabular}{|c|c|}
\hline $\mathrm{q}_{\mathrm{sat}, 1}$ & 379.065 \\
$\mathrm{~b}_{1}$ & 0.041 \\
\hline $\mathrm{v}_{1}$ & 1.083 \\
\hline $\mathrm{q}_{\mathrm{sat}, 2}$ & -500 \\
\hline $\mathrm{b}_{2}$ & 0.009 \\
\hline $\mathrm{v}_{2}$ & 0.777 \\
\hline $\mathrm{q}_{\mathrm{sat}, 3}$ & 0 \\
\hline $\mathrm{b}_{3}$ & 0 \\
\hline $\mathrm{v}_{3}$ & 0 \\
\hline
\end{tabular}

Figure S122. High pressure $\mathrm{CH}_{4}$ isotherm with fit (left) and corresponding fitting parameters (right) for $\mathrm{Zn}(\mathrm{bdc})_{2}$ dabco at $298 \mathrm{~K}$. 


\section{References}

1) Kapelewski, M. T.; Runčevski, T.; Tarver, J. D.; Jiang, H. Z. H.; Hurst, K. E.; Parilla, P. A.; Ayala, A.; Gennett, T.; FitzGerald, S. A.; Brown, C. M.; Long, J. R. Record High Hydrogen Storage Capacity in the Metal-Organic Framework $\mathrm{Ni}_{2}(m$-dobdc) at Near-Ambient Temperatures. Chem. Mater. 2018, 30, 81798189.

2) Furukawa, H.; Miller, M. A.; Yaghi, O. M. Independent verification of the saturation hydrogen uptake in MOF-177 and establishment of a bench-mark for hydrogen adsorption in metal-organic frameworks. $J$. Mater. Chem. 2007, 17, 3197-3204.

3) Ma, S.; Sun, D.; Simmons, J. M.; Collier, C. D.; Yuan, D.; Zhou, H. C. Metal-Organic Framework from an Anthracene Derivative Containing Nanoscopic Cages Exhibiting High Methane Uptake. J. Am. Chem. Soc. 2008, 130, 1012-1016.

4) Rallapalli, R.; Patil, D.; Prasanth, K. P.; Somani, R. S.; Jasra, R. V.; Bajaj, H. C. An alternative activation method for the enhancement of methane storage capacity of nanoporous aluminium terephthalate, MIL-53(Al). J. Porous Mater. 2010, 17, 523-528.

5) Charles, C. D.; Bloch, E. D. High-pressure methane storage and selective gas adsorption in a cyclohexane-functionalised porous organic cage. Supramol. Chem. 2019, 31, 508-513.

6) Wang, H.; Getzchmann, J.; Senkovska, I.; Kaskel, S. Structural transformation and high pressure methane adsorption of $\mathrm{Co}_{2}(1,4-\mathrm{bdc})_{2}$ dabco. Microporous Mesoporous Mater. 2008, 116, 653-657.

7) Mason, J. A.; Veenstra, M.; Long, J. R. Evaluating metal-organic frameworks for natural gas storage. Chem. Sci. 2014, 5, 32-51.

8) Llew, P. L.; Bourrelly, S.; Serre, C.; Vimont, A.; Daturi, M.; Hamon, L.; Weireld, G. D.; Chang, J. S.; Hong, D. Y.; Hwang, Y. K.; Jhung, S. H.; Férey, G. High Uptakes of $\mathrm{CO}_{2}$ and $\mathrm{CH}_{4}$ in Mesoporous MetalOrganic Frameworks MIL-100 and MIL-101. Langmuir 2008, 24, 7245-7250.

9) Lorzing, G. R.; Trump, B. A.; Brown, C. M.; Bloch, E. D. Selective Gas Adsorption in Highly Porous Chromium(II)-Based Metal-Organic Polyhedra. Chem. Mater. 2017, 29, 8583-8587.

10) Klein, N.; Hoffmann, H. C.; Cadiau, A.; Getzschmann, J.; Lohe, M. R.; Paasch, S.; Heydenreich, T.; Adil, K.; Senkovska, I.; Brunner E.; Kaskel, S. Structural flexibility and intrinsic dynamics in the $\mathrm{M}_{2}(2,6-$ ndc) $)_{2}($ dabco $)(\mathrm{M}=\mathrm{Ni}, \mathrm{Cu}, \mathrm{Co}, \mathrm{Zn})$ metal-organic frameworks. J. Mater. Chem. 2012, 22, 10303-10312.

11) Lorzing, G.; Gosselin, E. J.; Trump, B. A.; York, A. H. P.; Sturluson, A.; Rowland, C. A.; Yap, G. P. A.; Brown, C. M.; Simon, C. M.; Bloch, E. D. Understanding Gas Storage in Cuboctahedral Porous Coordination Cages. J. Am. Chem. Soc. 2019, 141, 12128-12138.

12) Sun, L. B.; Li, J. R.; Park, J.; Zhou, H. C. Cooperative Template-Directed Assembly of Mesoporous Metal-Organic Frameworks. J. Am. Chem. Soc. 2012, 134, 126-129.

13) Taggart, G. A.; Antonio, A. M.; Lorzing, G. R.; Yap, G. P. A.; Bloch, E. D. Tuning the Porosity, Solubility, and Gas-Storage Properties of Cuboctahedral Coordination Cages via Amide or Ester Functionalization. ACS Appl. Mater. Interfaces 2020, 12, 24913-24919.

14) Saha, D.; Deng, S. Structural Stability of Metal Organic Framework MOF-177. J. Phys. Chem. Lett. 2010, 1, 73-78.

15) Lee, J.Y.; Pan, L.; Huang, X.; Emge, T. J.; Li, J. A Systematic Approach to Building Highly Porous, Noninterpenetrating Metal-Organic Frameworks with a Large Capacity for Adsorbing $\mathrm{H}_{2}$ and $\mathrm{CH}_{4}$. Adv. Funct. Mater. 2011, 21, 993-998.

16) Lin, X.; Telepeni, I.; Blake, A. J.; Dailly, A.; Brown, C. M.; Simmons, J. M.; Zoppi, M.; Walker, G. S.; Thomas, K. M.; May, T. J.; Huvverstey, P.; Champness, N. R.; Schröder, M. High Capacity Hydrogen Adsorption in $\mathrm{Cu}(\mathrm{II})$ Tetracarboxylate Framework Materials: The Role of Pore Size, Ligand Functionalization, and Exposed Metal Sites. J. Am. Chem. Soc. 2009, 131, 2159-2171.

17) Deegan, M. M.; Ahmed, T. S.; Yap, G. P. A.; Bloch, E. D. Structure and redox tuning of gas adsorption properties in calixarene-supported Fe(II)-based porous cages. Chem. Sci. 2020, 11, 5273-5279.

18) Antonio, A. M.; Rosenthal, J.; Bloch, E. D. Electrochemically Mediated Syntheses of Titanium(III)Based Metal-Organic Frameworks. J. Am. Chem. Soc. 2019, 141, 11383-11387.

19) Sohail, M.; Yun, Y. N.; Lee, E.; Kim, S. K.; Cho, K.; Kim, J. N.; Kim, T. W.; Moon, J. H.; Kim, H. Cryst. Growth Des. 2017, 17, 1208-1213.

20) Decker, G. E.; Stillman, Z.; Attia, L.; Fromen, C. A.; Bloch, E. D. Controlling Size, Defectiveness, and Fluorescence in Nanoparticle UiO-66 through Water and Ligand Modulation. Chem. Mater. 2019, 31, 4831-4839.

21) Katz, M. J.; Brown, Z. J.; Colón, Y. J.; Siu, P, W.; Scheidt, K. A.; Snurr, R. Q.; Hupp, J. T.; Farha, O. K. A facile synthesis of UiO-66, UiO-67 and their derivatives. Chem. Commun. 2013, 49, 9449-9451. 
22) Lee, J. Y.; Olson, D. H.; Pan, L.; Emge, T. J.; Li, J. Microporous Metal-Organic Frameworks with High Gas Sorption and Separation Capacity. Adv. Funct. Mater. 2007, 17, 1255-1262.

23) Senkovska, I.; Kaskel, S. High pressure methane adsorption in the metal-organic frameworks $\mathrm{Cu}_{3}(\mathrm{btc})_{2}, \mathrm{Zn}_{2}(\mathrm{bdc})_{2} \mathrm{dabco}$, and $\mathrm{Cr}_{3} \mathrm{~F}\left(\mathrm{H}_{2} \mathrm{O}\right)_{2} \mathrm{O}(\mathrm{bdc})_{3}$. Microporous Mesoporous Mater. 2008, 112, 108-115. 